Алгебра и анализ

Том. 17 (2005), вып. 5
St. Petersburg Math. J.

Vol. 17 (2006), No. 5, Pages 699-744

S 1061-0022(06)00926-5

Article electronically published on July 20, 2006

\title{
BEURLING-MALLIAVIN MULTIPLIER THEOREM: THE SEVENTH PROOF
}

\author{
J. MASHREGHI, F. L. NAZAROV, AND V. P. HAVIN \\ In fond memory of Ol'ga Aleksandrovna Ladyzhenskaya
}

\begin{abstract}
We present a new proof of the Beurling-Malliavin theorem, often called the "multiplier theorem", concerning the existence of a real-valued function on $\mathbb{R}$ with spectrum in a given (small) interval and with a given small majorant of the modulus. This proof pertains entirely to real analysis. It only involves elementary facts about the Hilbert transformation; neither complex variable methods nor potential theory is exploited. The heart of the proof is Theorem 2, which treats preservation of the Lipschitz property under the Hilbert transformation. We also include a short survey of earlier proofs of the Beurling-Malliavin theorem and its generalizations to model (coinvariant) subspaces of the Hardy space $H^{2}(\mathbb{R})$.
\end{abstract}

"...Yet the seventh proof of that exists, reliable beyond doubt! And it will be shown you in a while." $\mathrm{Bu}$, p. 453]

\section{$\S 0$. INTRODUCTION}

0.1. Our purpose in this (mainly expository) paper is to present a new, relatively simple and self-contained proof of the Beurling-Malliavin multiplier theorem. Sometimes it is called "The first Beurling-Malliavin theorem", and we shall use the symbol BM1 to refer to it. Theorem BM2, lying beyond the scope of this paper, will be touched upon in Subsection 3.3 (X.Y means Subsection Y of $\S \mathrm{X}$ ).

We shall reduce Theorem BM1 to a certain problem about the Hilbert transformation on the real line. The only new result in the present paper is Theorem 2 in Subsection 2.1, proved by F. L. Nazarov. The reduction of Theorem BM1 to that statement is contained in [HMII and, implicitly, in Koo2, p. 502]. Here we reproduce this reduction along the lines of HMII, but with great simplifications. In particular, our presentation is free of the so-called model subspaces of the Hardy space $H^{2}(\mathbb{R})$, which were the main topic of [HMI] and [HMII] (we shall talk about them separately in Subsection 3.5). We wanted to give a focused and generally accessible proof of Theorem BM1, avoiding anywhere near important references to other sources. Our paper is written for nonexperts. We only assume the knowledge of elementary properties of the Fourier transformation on $\mathbb{R}$. For "good" functions $f$, the Fourier transformation is defined by the formula

$$
\hat{f}(\xi)=\int_{\mathbb{R}} f(x) e^{-2 \pi i \xi x} d x
$$

2000 Mathematics Subject Classification. Primary 42A50, 30D55.

Key words and phrases. Fourier transform, spectrum, Hardy space, Paley-Wiener space, Hilbert transform, inner function, outer function, logarithmic integral, Beurling-Malliavin theorem.

This work was supported by RFBR (grant no. 01-01-00377) and by "Scientific Schools" grant no. Sh-2266.2003.1. 
(which can be understood literally for $f \in L^{1}(\mathbb{R})$ and via the Plancherel theorem for $\left.f \in L^{2}(\mathbb{R})\right)$. We also need the notion of the spectrum of $f$. This is the set

$$
\operatorname{spec} f=\{\xi \in \mathbb{R}: \hat{f}(\xi) \neq 0\}
$$

(for $f \in L^{2}(\mathbb{R})$ the spectrum is defined up to a set of measure zero).

0.2. The time is ripe, however, for stating Theorem BM1. Despite the title of the paper, the term "multiplier" is not going to appear immediately. We shall explain it later, in Subsection 3.1.

Theorem BM1. Let $\omega$ be a continuous function defined on $\mathbb{R}$ and satisfying

$$
0<\omega \leq 1, \quad \int_{\mathbb{R}} \frac{\log \omega(x)}{1+x^{2}} d x>-\infty .
$$

If $\log \omega$ satisfies the Lipschitz condition, i.e.,

$$
\left|\log \omega\left(x^{\prime}\right)-\log \omega\left(x^{\prime \prime}\right)\right| \leq K\left|x^{\prime}-x^{\prime \prime}\right|
$$

for some $K>0$ and all $x^{\prime}, x^{\prime \prime} \in \mathbb{R}$, then for every $\sigma>0$ there is a nonzero function $f \in L^{2}(\mathbb{R})$ such that spec $f$ is included in a segment of length $\sigma$ and

$$
|f(x)| \leq \omega(x) \quad \text { for all } x \in \mathbb{R} .
$$

The class $L^{2}(\mathbb{R})$ is chosen here for definiteness and convenience. The essence of the theorem would not change if we were to replace this class by $L^{\infty}(\mathbb{R})$ (understanding the spectrum in the distributional sense) or, for instance, by $C^{\infty}(\mathbb{R}) \cap L^{1}(\mathbb{R})$.

0.3. The first acquaintance with this statement is unlikely to impress a nonspecialist. Its depth and importance will be discussed thoroughly in Subsections 0.5, 1.1, 1.3, and 3.2. Now we only note that, since its first appearance in BM1 in 1962, this theorem (apparently, designed for the needs of the celebrated Second Theorem BM2 about the completeness of a system of harmonics) has been a subject of permanent attention of analysts.

We confess that it is more for effect (see the epigraph) than for precise counting that we employed the numeral "seventh" in the title. Yet, definitely, at least six proofs of Theorem BM1 had been known before, and the texts treating this result in various ways run to hundreds of pages (see [BM1, Koo1, Koo2, Koo3, Koo5, DeB, HJ, Mal] and also the reference lists in Koo2, Koo3]).

There is something attractive in Theorem BM1 beyond its statement, which makes analysts reinterpret it again and again.

0.4. Many basic facts of real analysis were discovered with the help of complex tools. We mention, e.g., the Carleman-Ostrowski quasianalyticity theorem, convergence a.e. on the line or the circle of Cauchy-type singular integrals with $L^{p}$-density, and the entire theory of the Hardy classes $H^{p}$. In such situations, people often strove for elimination of external tricks, i.e., for decomplexification of the proofs, in order to clarify the true nature of the phenomena under study. Usually, this led to dramatic improvements rather than to mere clarification. Among the arguments initially involving complex analysis and made real afterwards, we can name Bang's proof of quasianalyticity theorems, the entire modern theory of singular integral operators (it originated in Lusin's paper $[\mathrm{L}$, now forgotten), activity in the spirit of the "abstract function theory" BK, $\mathrm{Hof}$, Gam, $\mathrm{Ru}$ ] (fashionable in the 1960s and 1970s), which, in particular, made it possible to carry many important statements about functions on the line $\mathbb{R}$ and the unit circle $\mathbb{T}$ over to a general group-theoretic setting, and, finally, the real-variable theory of Hardy classes $H^{p}$, which tore off the complex analysis mask from the very definition of them. Largely, 
the present paper falls within the same trend. The desire to "decomplexify" Theorem BM1 has highly influenced our attitude towards this remarkable result.

0.5. In many writings, Theorem BM1 is perceived as a statement within complex analysis (more precisely, the theory of entire functions, see the discussion in Subsection 3.1 below). We prefer a different stance and remain within real-variable methods. From the outset, we treat Theorem BM1 as a fact of harmonic analysis on $\mathbb{R}$ and relate it to the heuristic "uncertainty principle": "a nonzero function and its Fourier transform cannot be too small simultaneously". Theorem BM1 provides a tool to overcome some prohibitions imposed by this principle and, despite it, to guarantee the existence of (and in this paper even to actually construct) a nonzero function with a spectrum of "small" width and with a given "small" majorant $\omega$ - its smallness is only restricted by the convergence of the logarithmic integral

$$
\mathcal{L}(\omega)=\frac{1}{\pi} \int_{\mathbb{R}} \frac{\log \omega(x)}{1+x^{2}} d x
$$

in combination with a sort of level behavior of $\omega$. Neither in the statement of Theorem BM1 nor anywhere in the course of its proof do we resort to functions of a complex variable and to accompanying methods. At no point shall we employ harmonic or sub(super-) harmonic functions, or potential theory, or conformal mappings, or the Dirichlet integral ("energy") - in other words, we use none among the ingredients of previous proofs. Our only tool will be the Hilbert transformation which, unlike complex analysis or potential theory, is an indispensable and primary constituent of Fourier analysis on the line $\mathbb{R}$.

0.6. In our opinion, another merit of the proof given below is that it is economical in employing ideas and methods: nothing beyond the elementary theory of singular integral operators is used. This makes the argument relatively short and direct, at least as compared to some known proofs.

Our proof does not involve procedures whose realizability is mere words without actual deeds (such as the search for an extremum of a functional on the basis of the weak compactness of the ball of a Hilbert space, or construction of a conformal mapping of a domain with complicated boundary onto a half-plane, or calculation of the smallest superharmonic majorant for a given family, etc.). The required function $f$ will be given by an explicit formula, "in quadratures" so-to-say. True, our proof involves a procedure (sometimes rather painful and burdensome) of preliminary regularization of the majorant $\omega$, but even this can be done quite "explicitly" in specific cases. Furthermore, our approach is applicable as it is to analogs of Theorem BM1 for a wide class of the socalled "model subspaces" (see Subsection 3.5 below), and there its possibilities seem to be very far from exhausted.

0.7. It should be mentioned that in no way does the above discussion attempt to depreciate or "cancel" any of the previous proofs of Theorem BM1. Though we perceive our approach to be nearer to "geodesic" and to provide a quicker way to the target, at the same time ("simplicity is worse than a crime"!) it eliminates many boons and links interesting for their own sake. The classical paper [BM1] deserves a special mention here: the roundabout way chosen there passes, however, through a remarkable result, which is based on energy methods (see Subsection 3.4 below) and does not fit (for the time being?) into our pattern.

Every one of the available proofs reaches the function $f$ (see $(0.2)$ ) in its own manner, with its own (dis)advantages. Every one brings about incidental results, quite useful 
beyond the scope of Theorem BM1. We think that in our proof the role of such a result can be played by Theorem 2 (Subsection 2.1).

0.8. The paper is organized as follows. In $\S 1$ we discuss the setting of the problem, present necessary facts about the Hilbert transformation, and then reduce Theorem BM1 to a certain problem about preservation of the Lipschitz property under this transformation. That problem is solved in $\S 2$. In the last section, $\S 3$, we comment on the proofs in $\S \S 1$ and 2, give some historical information, review generalizations to model subspaces, and indicate some references.

\section{§1. Discussion of the PRoblem SETting, PRELIMINARIES, AND THE BEGINNING OF THE PROOF OF THEOREM BM1}

\subsection{A version of the uncertainty principle for functions with semibounded spectrum. Put}

$$
H^{2}=H^{2}(\mathbb{R})=\left\{f \in L^{2}(\mathbb{R}): \operatorname{spec} f \subset[0,+\infty)\right\} .
$$

The reader experienced in complex analysis is free to use the Paley-Wiener theorem [HJ, p. 172] and to identify a function in $H^{2}$ with the corresponding function analytic in the upper half-plane $\mathbb{C}_{+}$and belonging to the Hardy class $H^{2}\left(\mathbb{C}_{+}\right)$, but we need not perceive things this way, nor do we need the very acquaintance with the class $H^{2}\left(\mathbb{C}_{+}\right)$. The following version of the uncertainty principle can be proved without leaving the real line $\mathbb{R}$; see [HJ, pp. 32-36].

Theorem. If $f \in H^{2}$ and $\mathcal{L}(f)=\int_{\mathbb{R}} \log |f| d P=-\infty$, then $f=0$.

Here $P$ is the Poisson measure on $\mathbb{R}$, i.e., $d P(x)=\frac{1}{\pi} \frac{d x}{1+x^{2}}$.

The relation $\mathcal{L}(f)=-\infty$ means that $|f|$ is "small". Indeed, $\log ^{+} x \leq x$ for $x>0$; therefore $\int_{\mathbb{R}} \log ^{+}|f| d P<+\infty$ whenever $f \in L^{1}(P)$ (a fortiori, for all $f \in L^{2}(P)$ ), and the relation $\mathcal{L}(f)=-\infty$ means that $\int_{\mathbb{R}} \log ^{-}|f| d P=+\infty$. The theorem says that the smallness of $f$ expressed by the condition $\mathcal{L}(f)=-\infty$ is incompatible with the spectral smallness imposed by the condition $f \in H^{2}$, i.e., with the total absence of the spectrum on $(-\infty, 0)$. A remarkable feature of this theorem (quite rare for similar statements) is its ultimate sharpness: an arbitrary nonnegative function $\omega \in L^{2}$ with finite logarithmic integral $\mathcal{L}(\omega)$ is the modulus of some $H^{2}$-function $f$; moreover, an $f \in H^{2}$ with $|f|=\omega$ can be given by an explicit formula (see Subsection 1.8 below).

1.2. Now we turn to bounded spectra in place of semibounded, i.e., to "band-limited functions" so popular among applied mathematicians. It is hardly possible to hope for an anywhere near efficient description of the moduli of such functions or for a discovery of a method to produce such functions with a given modulus. We shall have to simplify the problem substantially and, instead of functions with a spectrum of a given (finite) width and with a given modulus $\omega$, we shall only try to construct nonzero functions dominated by $\omega$ in modulus and having a spectrum of given width.

Consider the Paley-Wiener class

$$
P W_{\sigma / 2}=\left\{f \in L^{2}: \hat{f}=0 \text { off }\left[-\frac{\sigma}{2}, \frac{\sigma}{2}\right]\right\},
$$

i.e., the class of all $L^{2}$-functions with spectrum in the segment $\left[-\frac{\sigma}{2}, \frac{\sigma}{2}\right]$. The operator of multiplication by $e^{\pi i \sigma x}$ preserves the moduli of functions, and maps $P W_{\sigma / 2}$ isometrically onto the class

$$
H_{\sigma}^{2}=\left\{f \in L^{2}: \operatorname{spec} f \subset[0, \sigma]\right\} .
$$

This is the class to be dealt with in what follows. 
1.3. The statement of the problem solved (to a certain extent) by Theorem BM1 is suggested by the following definition.

A function $\omega$ defined on $\mathbb{R}$ is said to be $\sigma$-admissible if there exists a nonzero function $f \in H_{\sigma}^{2}$ with $|f| \leq \omega$. If $\omega$ is $\sigma$-admissible for every $\sigma$, it is called fully admissible.

This definition implies three tasks:

1 ) to give a description (as efficient as possible) of $\sigma$-admissible functions;

2 ) in case $\omega$ is $\sigma$-admissible, to learn how to construct functions $f \in H^{2}$ subordinate to $\omega$, i.e., satisfying $|f| \leq \omega$;

3 ) though a complete description of the moduli of functions in $H_{\sigma}^{2}$ is an inaccessible ideal, to try nonetheless to find a function $f \in H_{\sigma}^{2}$ with $|f| \leq \omega$ that imitates, at least to a slight extent, the behavior of $\omega$ (for example, to ensure a sort of lower estimate for $|f| / \omega)$.

In spite of its simplicity, the following remark will play a prominent role in our exposition: if $\omega, \omega_{1} \in C(\mathbb{R})$ are positive and $\omega_{1} \leq \omega$ (or at least $\varlimsup_{|x| \rightarrow \infty} \omega_{1}(x) / \omega(x)<+\infty$ ), then $\omega$ is $\sigma$-admissible whenever $\omega_{1}$ is.

We denote $\Omega=\log \frac{1}{\omega}$ (this notation will be used repeatedly in what follows). The conditions $0<\omega \leq 1, \mathcal{L}(\omega)>-\infty$ are equivalent to the conditions

$$
\Omega \geq 0, \quad \int_{\mathbb{R}} \Omega d P<+\infty .
$$

Observe that, since $H_{\sigma}^{2} \subset H^{2}$, the condition $\mathcal{L}(\omega)>-\infty$ is still necessary for $\omega$ to be $\sigma$-admissible. However, unlike the case of semibounded spectra, now it is very far from being sufficient. This explains the peculiarity of the problem in question and the importance of Theorem BM1, which tells us what else, besides the finiteness of the logarithmic integral $\mathcal{L}(\omega)$, makes $\omega \sigma$-admissible.

1.4. We are going to present quite a crude example of a continuous and everywhere positive function $\omega$ that is not $\sigma$-admissible for any $\sigma>0$, though $\mathcal{L}(\omega)>-\infty$.

For this we observe that if $f \in H_{\sigma}^{2}$ and $|f(x)| \leq C e^{-x}$ for $x>1$ with some $C>0$, then $\mathcal{L}(f)=-\infty$ and $f=0$. Next, observe that every function $f \in H_{\sigma}^{2}$ has a continuous derivative, and

$$
\left|f^{\prime}(x)\right|=\left|\int_{0}^{\sigma} \hat{f}(\xi) e^{2 \pi i \xi x} 2 \pi i \xi d \xi\right| \leq 2 \pi \sigma^{3 / 2}\|f\|_{L^{2}}
$$

for all $x \in \mathbb{R}$.

Now we introduce a monotone increasing sequence $\left\{x_{n}\right\}_{n>0}$ by recursion: $x_{0}=1$; $x_{n}-x_{n-1}=e^{-x_{n}}, n \geq 1$ (it is easily seen that $x_{n}$ is uniquely determined by the latter equation, and $\left.x_{n}>x_{n-1}\right)$. Note that if $x_{n}<B$, then $x_{n}-x_{n-1}>e^{-B}$, so the number of terms of the sequence $\left\{x_{n}\right\}$ that do not exceed $B$ is at most $e^{B}(B-1)+1$, i.e., $x_{n} \underset{n \rightarrow \infty}{\longrightarrow}+\infty$. Suppose $\omega \in C(\mathbb{R})$ satisfies $0<\omega \leq 1$ and $\omega\left(x_{n}\right)=e^{-x_{n}}$. If $f \in H_{\sigma}^{2}$ and $|f| \leq \omega$, then on every segment $\left(x_{n-1}, x_{n}\right]$ we have

$$
\begin{aligned}
|f(x)| & \leq\left|f\left(x_{n}\right)\right|+\left\|f^{\prime}\right\|_{\infty}\left|x-x_{n}\right| \leq e^{-x_{n}}+2 \pi \sigma^{3 / 2}\|f\|_{L^{2}}\left(x_{n}-x_{n-1}\right) \\
& =\left(1+2 \pi \sigma^{3 / 2}\|f\|_{L^{2}}\right) e^{-x_{n}} \leq\left(1+2 \pi \sigma^{3 / 2}\|f\|_{L^{2}}\right) e^{-x} .
\end{aligned}
$$

The above remarks show that $f=0$, i.e., $\omega$ is not $\sigma$-admissible. On the other hand, the conditions imposed on $\omega$ are compatible with the finiteness of $\mathcal{L}(\omega)$. For instance, we may put $\omega \equiv 1$ off small intervals centered at the points $x_{n}$ and make $\omega$ linear on the right and the left half of every such interval. If the lengths $l_{n}$ of these intervals tend to zero so quickly that the series $\sum_{n \geq 0} l_{n} x_{n}$ converges, then $\log \omega$ belongs to $L^{1}$ and, $a$ fortiori, to $L^{1}(P)$. 


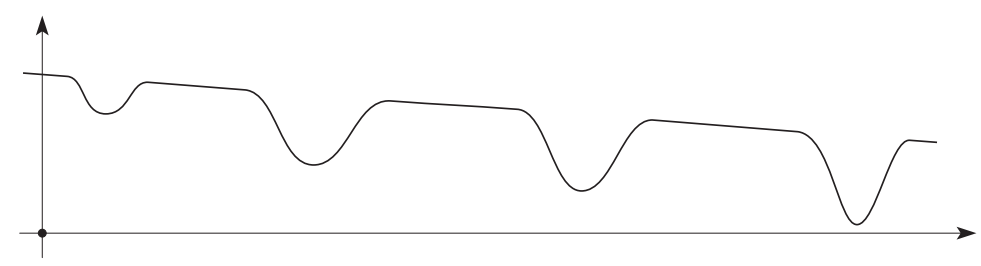

Figure 1.

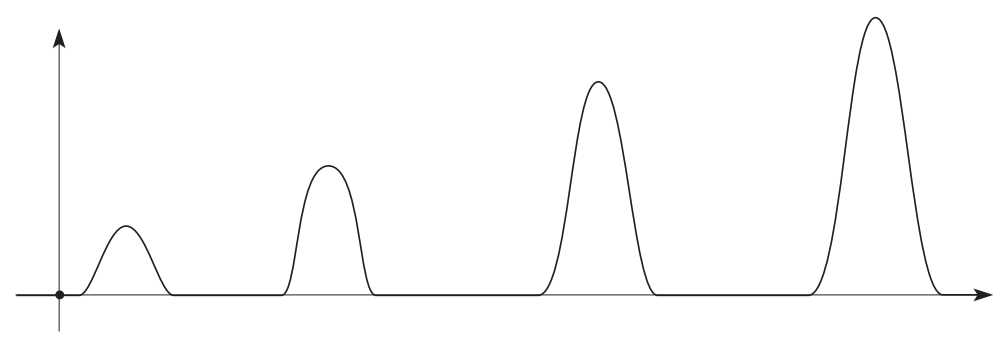

FIGURE 2.

1.5. In this example the majorant $\omega$ and the corresponding function $\Omega$ oscillate highly. Oscillation could be made not so rapid, but by Theorem BM1, the peaks of $\Omega$ must have unboundedly steep slopes if $\omega$ is not $\sigma$-admissible and $\Omega \in L^{1}(P)$. The following statement shows that, moreover, infinitely many oscillations must occur in this case.

Theorem. If $\omega$ is monotone decreasing on $[0,+\infty)$ and monotone increasing on $(-\infty, 0]$, and $\Omega \in L^{1}(P)$, then $\omega$ is fully admissible.

Several proofs of this theorem are known. They differ by the method of constructing a function $f \in H_{\sigma}^{2}$ that satisfies the inequality $|f| \leq \omega$; see [HJ, Koo1, HMI, GLO]. Yet another proof will be indicated in Subsection 3.6.9 below. Thus, only under some regularity assumptions about $\omega$ may the finiteness of the logarithmic integral $\mathcal{L}(\omega)$ ensure that $\omega$ is $\sigma$-admissible. A typical obstruction to the $\sigma$-admissibility of a majorant $\omega$ with $\mathcal{L}(\omega)<\infty$ is shown in Figure 1: the graph has numerous pits that deepen unboundedly at infinity.

These pits give rise to "hills" of unbounded height on the graph of $\Omega=\log \frac{1}{\omega}$ (Figure 2 ). Theorem BM1 shows that if the sizes of the hills obey the condition $\int_{\mathbb{R}} \Omega d P<+\infty$ and the steepness of their slopes is uniformly bounded, then $\omega$ is fully admissible.

1.6. One more reformulation of Theorem BM1. Here we restate that theorem in a seemingly stronger form. In fact, it will be shown immediately that the two statements are equivalent.

Theorem BM1. Suppose a positive bounded function $\omega$ on $\mathbb{R}$ satisfies the inequalities $\mathcal{L}(\omega)=\int_{\mathbb{R}} \log \omega d P>-\infty$ and

$$
\sup \left\{\frac{\omega(x)}{\omega(y)}:|x-y| \leq 1\right\}<+\infty .
$$

Then $\omega$ is fully admissible.

It is the second condition of the theorem that was violated radically in Subsection 1.4 (while the first condition was fulfilled). In terms of $\Omega$, this second condition means that $|\Omega(x)-\Omega(y)| \leq C$ for all $x, y \in \mathbb{R}$ with $|x-y| \leq 1$. This is so indeed if $\Omega$ is uniformly continuous and, a fortiori, if $\Omega$ is Lipschitzian. Therefore, the new statement really 
improves the initial one. On the other hand, if $\omega$ satisfies the second condition in the new statement, it is easy to construct a comparable function $\omega_{1}$ such that $\Omega_{1}=\log \frac{1}{\omega_{1}}$ is Lipschitzian. Indeed, put $\Omega=\log \frac{1}{\omega}$ as before, and choose a function $\varphi \in C^{\infty}(\mathbb{R})$ such that $\varphi \geq 0, \varphi \equiv 0$ off $[-1,1]$, and $\int_{\mathbb{R}} \varphi=1$. Then take $\Omega_{1}=\Omega * \varphi$. For every $x \in \mathbb{R}$ we have $\left|\Omega_{1}(x)-\Omega(x)\right| \leq \int_{-1}^{1}|\Omega(x-y)-\Omega(x)||\varphi(y)| d y \leq C$ and $\left|\Omega_{1}^{\prime}(x)\right|=$ $\left|\left(\Omega * \varphi^{\prime}\right)(x)\right|=\left|\int_{-1}^{1}(\Omega(x-y)-\Omega(x)) \varphi^{\prime}(y) d y\right| \leq C \int_{-1}^{1}\left|\varphi^{\prime}(y)\right| d y$. We define $\omega_{1}=e^{-\Omega_{1}}$. Since the ratio $\frac{\omega_{1}}{\omega}$ is uniformly bounded, we see that $\omega$ is $\sigma$-admissible whenever $\omega_{1}$ is. But $\Omega_{1}=\log \frac{1}{\omega_{1}}$ is a Lipschitz function, and if $\Omega \in L^{1}(P)$, then $\Omega_{1} \in L^{1}(P)$. Therefore, the initial statement of Theorem BM1 shows that $\omega_{1}$ is fully admissible. Thus, the new formulation of Theorem BM1 can be deduced from the initial one.

It should be noted that $\Omega_{1} \in C^{\infty}(\mathbb{R})$. Thus, we may add the assumption $\omega \in C^{\infty}(\mathbb{R})$ to Theorem BM1 without loss of generality. However, rather, the value of this observation is purely psychological.

At this point, we stop temporarily the discussion of Theorem BM1 and pass to its proof. As has already been mentioned, our main tool will be the Hilbert transformation. The next subsection is devoted entirely to its definition and basic properties. The material of that subsection is perfectly classical and can be found in most of the standard textbooks on harmonic analysis; therefore, we restrict ourselves to a cursory glance. Missing technical details can be found, e.g., in [Koo4, Ga, $\mathrm{Pr}, \mathrm{Ti}$.

1.7. Largely, the Hilbert transformation will be used here to control the spectrum of functions. In accordance with this, we start with a spectral definition: the Hilbert transformation is the operator $h_{0}$ acting by the formula

$$
h_{0}(f)=(-i \cdot \operatorname{sgn} \xi \cdot \hat{f})^{\vee}
$$

for functions $f$ defined on $\mathbb{R}$ (here $\vee$ indicates the inverse Fourier transform). This definition works perfectly for $f \in L^{2}(\mathbb{R})$ and implies immediately the following property, which will be most important for us:

$$
\operatorname{spec}\left(f+i h_{0}(f)\right) \subset[0,+\infty) .
$$

No less important is the inversion formula

$$
h_{0}\left(h_{0}(f)\right)=-f
$$

which is also a direct consequence of the definition. The basic properties of the Fourier transformation imply that the Hilbert transformation commutes with translations and with positive dilations, that is, putting

$$
f_{\lambda}(x)=f(\lambda x)(\lambda>0) \quad \text { and } \quad f^{\tau}(x)=f(x+\tau)(\tau \in \mathbb{R}),
$$

we have

$$
h_{0}\left(f_{\lambda}\right)=\left(h_{0}(f)\right)_{\lambda} \quad \text { and } \quad h_{0}\left(f^{\tau}\right)=\left(h_{0}(f)\right)^{\tau} .
$$

We need yet another observation. If $f \in C^{\infty}(\mathbb{R})$ and $f \equiv 0$ off a bounded interval, then $h_{0}(f) \in C^{\infty}$ and $\left(h_{0}(f)\right)^{\prime}$ is uniformly bounded. This is an immediate consequence of the fact that $\hat{f}$ decays rapidly.

The Hilbert transformation can be represented as a singular integral. To deduce the formula, we observe that $h_{0}(f)=\lim _{\varepsilon \rightarrow 0+} h_{\varepsilon}(f)$, where

$$
\begin{aligned}
h_{\varepsilon}(f) & =\left(-i \operatorname{sgn} \xi e^{-2 \pi \varepsilon|\xi|} \cdot \hat{f}\right)^{\vee} \\
& =\left(-i \operatorname{sgn} \xi e^{-2 \pi \varepsilon|\xi|}\right)^{\vee} * f=\frac{x}{\pi\left(\varepsilon^{2}+x^{2}\right)} * f .
\end{aligned}
$$


Passing to the limit, we obtain

$$
h_{0}(f)(x)=\frac{1}{\pi} \int_{\mathbb{R}} \frac{f(t)}{x-t} d t .
$$

Here the integral is understood as the principal value, i.e., as $\lim _{\varepsilon \rightarrow 0+} \int_{\{|x-t|>\varepsilon\}}$. The above formula allows us to extend the definition of the Hilbert transform $h_{0}(f)$ to the functions in $L^{1}\left(\frac{d t}{1+|t|}\right)$. If $f$ belongs to this class, then the integral $f_{\mathbb{R}} \frac{f(t)}{x-t} d t$ converges at infinity, and it can be shown that for almost every $x \in \mathbb{R}$ the principal value of this integral exists and is finite at $x$. But we shall deal only with functions differentiable everywhere except at points forming a discrete set, and in this case the existence of the principal value at every differentiability point is obvious:

$$
h_{0}(f)(x)=\frac{1}{\pi} \oint \frac{f(t)}{x-t} d t=\frac{1}{\pi} \int_{\{|x-t|<1\}} \frac{f(t)-f(x)}{x-t} d t+\frac{1}{\pi} \int_{\{|x-t| \geq 1\}} \frac{f(t)}{x-t} d t .
$$

All properties of the Hilbert transformation proved above for the class $L^{2}(\mathbb{R})$ can easily be extended to the (wider) class $L^{1}\left(\frac{d t}{1+|t|}\right)$. The only subtlety is that in the inversion formula it should be assumed that both $f$ and $h_{0}(f)$ are in $L^{1}\left(\frac{d t}{1+|t|}\right)$. Unfortunately, the class $L^{1}\left(\frac{d t}{1+|t|}\right)$ is still too narrow for our purposes. We need to extend the definition of the Hilbert transform to functions belonging to $L^{1}(P)$. In this situation the integral $f_{\mathbb{R}} \frac{f(t)}{x-t} d t$ may diverge at infinity, which forces us to slightly modify the Cauchy kernel $\frac{1}{x-t}$. We put

$$
\tilde{f}(x)=h(f)(x)=\frac{1}{\pi} \oint_{\mathbb{R}} f(t)\left(\frac{1}{x-t}+\frac{t}{t^{2}+1}\right) d t .
$$

Note that the modified Cauchy kernel $\frac{1}{x-t}+\frac{t}{t^{2}+1}$ decays at infinity as $\frac{1}{t^{2}}$ and, therefore, the integral defining $h(f)$ converges at infinity for every $f \in L^{1}(P)$. For $f \in L^{2}(\mathbb{R})$ (and even for $\left.f \in L^{1}\left(\frac{d t}{1+|t|}\right)\right)$ the new definition coincides with the initial one up to a constant summand:

$$
h(f)(x)=\frac{1}{\pi} \int_{\mathbb{R}} \frac{f(t)}{x-t} d t+\frac{1}{\pi} \int_{\mathbb{R}} f(t) \frac{t}{t^{2}+1} d t=h_{0}(f)+\text { const. }
$$

It follows that, for $f \in L^{2}(\mathbb{R})$, we still have $\operatorname{spec}(f+i h(f)) \subset[0,+\infty)$ (now this inclusion should be understood in the distributional sense). Observing that the Hilbert transform of a constant function is identically zero, we obtain the inversion formula:

$$
h(h(f))=h\left(h_{0}(f)+\text { const }\right)=h\left(h_{0}(f)\right)=h_{0}\left(h_{0}(f)\right)+\text { const }=-f+\text { const },
$$

which is valid for $f \in L^{2}(\mathbb{R})$. By standard (though fairly subtle) approximation arguments, the above two properties can be extended to $f \in L^{1}(P)$ under the assumption that $h(f)$ also belongs to $L^{1}(P)$. A suitable variant of the inversion formula for $h$ will be given in Subsection 3.6.2.

A constant function has zero Hilbert transform. It happens to be convenient to agree that the Hilbert transform itself of a function $f \in L^{1}(P)$ is also defined up to a constant summand. Sometimes we use the symbol $\tilde{f}$ to signalize that this agreement is adopted at the moment: $\tilde{f}=h(f)+c, c \in \mathbb{R}$.

Some additional facts about the Hilbert transformation will be described when they are needed (in §2). Now we present another classical result without proof. We shall not use it seriously, but it will be helpful in the discussion of a detail in the proof of Theorem BM1. 
Kolmogorov theorem. Let $f \in L^{1}(P)$. Then

$$
P(\{x \in \mathbb{R}:|h(f)(x)|>a\})=o\left(\frac{1}{a}\right) \quad \text { as } a \rightarrow+\infty .
$$

1.8. Now, we pass to the proof itself of Theorem BM1. Our argument is a development of the well-known method employed to construct a function with semibounded (for instance, nonnegative) spectrum and given modulus. We mean the so-called "outer function" Ext $\omega$ corresponding to a function $\omega=e^{-\Omega} \in L^{2}, \omega \geq 0$ :

$$
\operatorname{Ext} \omega=a \omega e^{i \widetilde{\log \omega}}=a e^{-(\Omega+i \widetilde{\Omega})},
$$

where $a$ is an arbitrary complex number with unit modulus. Here it is assumed that $\Omega=\log \frac{1}{\omega} \in L^{1}(P)$, so that the Hilbert transform $\widetilde{\Omega}$ makes sense. Clearly, $|\operatorname{Ext} \omega|=\omega$. It is important that the spectrum of $f=\operatorname{Ext} \omega$ is nonnegative:

$$
\operatorname{spec} \operatorname{Ext} \omega \subset[0,+\infty) \text {. }
$$

This observation is made within a complex variable mode of thought, but in fact complex analyticity is not required for that. At the engineer's level of rigor, this fact can be explained as follows: the rule of adding spectra says that $\operatorname{spec}\left(g_{1} g_{2}\right)$ is nonnegative whenever so are the spectra of $g_{1}$ and $g_{2}$; next, by the main property of Hilbert transforms, the spectrum of the function $\varphi=-\Omega-i \widetilde{\Omega}$ (and, with it, of all functions $\left(1+\frac{\varphi}{n}\right)^{n}$, $n=1,2, \ldots)$ is nonnegative, and a passage to the limit shows that $\operatorname{spec} e^{\varphi} \subset[0,+\infty)$. A mathematically rigorous argument based on the same ideas can be found in Subsection 3.6.4.

1.9. Yet we need a function $f$ in $H_{\sigma}^{2}$ rather than merely in $H^{2}$. In recompense, in place of the identity $|f|=\omega$, it suffices to ensure the inequality $|f| \leq \omega$, so we meet with a blend of complications and simplifications.

We start with restating the definition of $H_{\sigma}^{2}$ : the fact that $f \in H_{\sigma}^{2}$ means that the functions $f$ and $\bar{f} e^{2 \pi i \sigma x}$ both lie in $H^{2}$. Indeed, $\bar{f} e^{2 \pi i \sigma x} \in H^{2}$ if and only if spec $f \subset$ $(-\infty, \sigma]$. The following remark is equally simple.

If $\psi \geq 0, \psi \in L^{2}, \mathcal{L}(\psi)>-\infty$, and the function $\psi^{2} e^{2 \pi i \sigma x}$ is outer, then $\psi=|f|$ for some function $f \in H_{\sigma}^{2}$.

Proof. Let $Q$ be an outer function with $|Q|=\psi ; Q \in H^{2}$. Then the function $Q^{2}$ is also outer and $\left|Q^{2}\right|=\psi^{2}$. But, by assumption, $\psi^{2} e^{2 \pi i \sigma x}$ is yet another outer function and its modulus is also equal to $\psi^{2}$. Therefore, $\psi^{2} e^{2 \pi i \sigma x}=c Q^{2}$ for some $c \in \mathbb{C},|c|=1$. But $\psi^{2}=Q \bar{Q}$ and, dividing by $Q$, we deduce that $\bar{Q} e^{2 \pi i \sigma x}=c Q \in H^{2}$. Consequently, $Q \in H_{\sigma}^{2}$.

This statement is a special case of a theorem due to Dyakonov (see Subsection 3.5.4).

1.10. So, if the function $\omega^{2} e^{2 \pi \sigma x i}$ turns out to be outer, then $\omega$ coincides with the modulus of the function Ext $\omega$ in $H_{\sigma}^{2}$. But we are interested only in the $\sigma$-admissibility of $\omega$, so it is not required that some function in $H_{\sigma}^{2}$ have modulus precisely $\omega$. Instead of the identity $|f|=\omega$, we would be contented with

$$
|f|=m \omega
$$

where $f \in H_{\sigma}^{2}$ and $m$ is a certain nonzero bounded function. Applying the above criterion to $\psi=m \omega$, we see that if $m \in L^{\infty}, m \geq 0, \mathcal{L}(m)>-\infty, m \omega \in L^{2}$, and the function $m^{2} \omega^{2} e^{2 \pi i \sigma x}$ is outer, then $m \omega$ is the modulus of some function in $H_{\sigma}^{2}$ (namely, of the function $f=\operatorname{Ext} m \omega$ ). Consequently, in order to prove that $\omega$ is $\sigma$-admissible, it suffices to find a nonnegative function $m \in L^{\infty}$ such that $\mathcal{L}(m)>-\infty, m \omega \in L^{2}$, and $m^{2} \omega^{2} e^{2 \pi i \sigma x}$ is an outer function. Recalling what an outer function is and denoting $M=\log \frac{1}{m}$, 


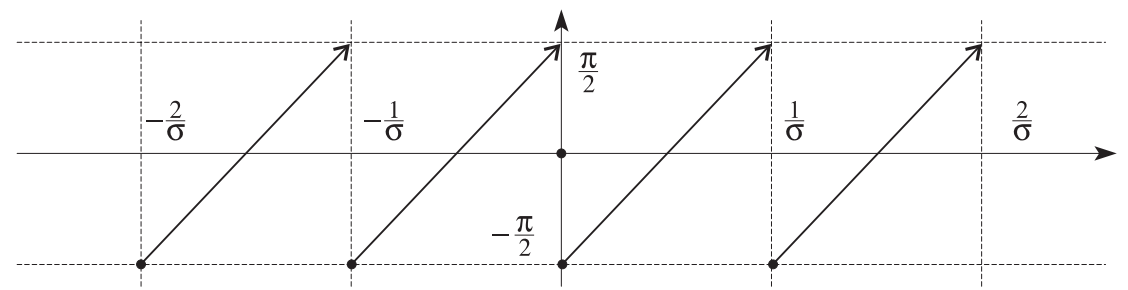

FiguRE 3.

$\Omega=\log \frac{1}{\omega}$, we see that it suffices to find a lower bounded solution $M \in L^{1}(P)$ of the congruence

$$
-2 h(M)-2 h(\Omega)+2 \pi \sigma x \equiv \text { const } \quad(\bmod 2 \pi)
$$

or, which is the same, of the congruence

$$
-h(M) \equiv \pi \sigma x+h(\Omega)+\mathrm{const} \quad(\bmod \pi),
$$

such that $e^{-M} \omega \in L^{2}$. We note that the identity

$$
-h(M)=\pi \sigma x+h(\Omega)+\text { const }
$$

cannot be true for any $M \in L^{1}(P)$. This follows from the Kolmogorov theorem: we have $P(\{\mid h(M)+h(\Omega)+$ const $\mid>a\})=o\left(\frac{1}{a}\right)$, but $P(\{|\pi \sigma x|>a\}) \asymp \frac{1}{a}$ as $a \rightarrow+\infty$. Therefore, the congruence $(*)$ with an unknown function $M$ can be resolved only at the expense of choosing a nontrivial function $k: \mathbb{R} \rightarrow \mathbb{Z}$ with

$$
-h(M)=\pi \sigma x+h(\Omega)-\pi k+\text { const. }
$$

We agree to view $h(\Omega)$ as a "small perturbation" for the linear function $\pi \sigma x$ and try to find out what would happen if the perturbation were totally absent. In this case it is natural to take the risk of choosing $k$ that counterbalances the growth of the function $\pi \sigma x$ entirely, that is, to ensure the inequality

$$
0 \leq \pi \sigma x-\pi k(x)<\pi \quad \text { for every } x \in \mathbb{R} .
$$

This means that we take $k(x)=[\sigma x]$. Then the right-hand side of the equation turns into $s_{\sigma}+$ const, where $s_{\sigma}$ is the saw-like function having period $\frac{1}{\sigma}$, growing linearly on the interval $[0,1 / \sigma)$, and mapping this interval onto $\left[-\frac{\pi}{2}, \frac{\pi}{2}\right)$ (see Figure 3 ).

In order to find a solution $M$ of the equation

$$
-h(M)=s_{\sigma}+\text { const, }
$$

it is natural to use the inversion formula, which yields

$$
M=h\left(s_{\sigma}+\text { const }\right)=-\log |\sin \pi \sigma x|+\text { const }
$$

(see Subsection 3.6.2). Disregarding the constant (which disappears in any case under taking the Hilbert transform), we obtain $m=m_{\sigma}=|\sin \pi \sigma x|$. Putting $f_{\sigma}=\operatorname{Ext} m_{\sigma}$, we obtain a bounded function with spectrum in $[0, \sigma]$. It can be shown that $f_{\sigma}=$ $c \sin \pi \sigma x e^{i \pi \sigma x}$. Note that $f_{\sigma} / x \in H_{\sigma}^{2}$. The functions $f \in H_{\sigma}^{2}$ with $|f| \leq \omega$ to be constructed in the general case (i.e., when $\Omega \not \equiv 0$ ) will resemble $f_{\sigma} / x$.

1.11. Now, we try to proceed similarly in the presence of the perturbation $h(\Omega)$. As before, we choose $k(x)=\left[\sigma x+\frac{h(\Omega)}{\pi}\right]$ in order to compensate for the growth of $\pi \sigma x+h(\Omega)$ maximally. We arrive at the equation

$$
-h(M)=s+\text { const, }
$$


where $s=\pi \sigma x+h(\Omega)-\pi k(x)-\frac{\pi}{2} \in L^{\infty},\|s\|_{L^{\infty}} \leq \frac{\pi}{2}$. Solving it with the help of the inversion formula, we get

$$
M=h(s) .
$$

If we could show that the function $M$ defined in this way is bounded below and belongs to $L^{1}(P)$, we would verify that $\omega$ is $\sigma$-admissible. We have

$$
\begin{aligned}
M(x)= & \frac{1}{\pi} \int_{\mathbb{R}} s(t)\left(\frac{1}{x-t}+\frac{t}{t^{2}+1}\right) d t \frac{1}{\pi} \int_{\{|x-t|<1\}} \frac{s(t)}{x-t} \\
& +\frac{1}{\pi} \int_{\{|x-t|<1\}} s(t) \frac{t}{t^{2}+1} d t+\frac{1}{\pi} \int_{\{|x-t| \geq 1\}} s(t)\left(\frac{1}{x-t}+\frac{t}{t^{2}+1}\right) d t \\
= & J_{1}+J_{2}+J_{3} .
\end{aligned}
$$

Clearly, the integral $J_{2}$ is bounded: $\left|J_{2}\right| \leq \frac{1}{\pi} \cdot 2 \cdot\|s\|_{L^{\infty}} \cdot \frac{1}{2} \leq \frac{1}{2}$. Generally speaking, the integral $J_{3}$ may grow as $x$ increases, but this growth is very slow:

$$
\begin{aligned}
\left|J_{3}\right| & \leq \frac{1}{\pi}\|s\|_{\mathrm{L}^{\infty}} \int_{\{|x-t| \geq 1\}}\left|\frac{1}{x-t}+\frac{t}{t^{2}+1}\right| d t \\
& \leq B\|s\|_{L^{\infty}} \log (|x|+2) \leq \frac{\pi}{2} B \log (|x|+2),
\end{aligned}
$$

where $B>0$ is a universal constant. So, the integral $J_{1}$ presents the main problem. Writing it in the form

$$
J_{1}=\frac{1}{\pi} \int_{\{|x-t| \leq 1\}} \frac{s(t)-s(x)}{x-t} d t,
$$

we see that it is bounded from below in two cases: first, if $s$ is Lipschitzian (then $\left|J_{1}\right| \leq$ $\frac{2}{\pi}\left\|s^{\prime}\right\|_{L^{\infty}}$ ), and second, if $s$ is monotone decreasing, and then $J_{1} \geq 0$. Thus, $J_{1}$ is bounded below if $s$ is the difference of a Lipschitz function and a monotone decreasing function. We remind the reader that $s=\pi \sigma x+h(\Omega)(x)-\pi k(x)$, where $k(x)=\left[\sigma x+\frac{h(\Omega)(x)}{\pi}\right]$. This shows that $J_{1}$ is bounded below provided $\pi \sigma x+h(\Omega)(x)$ is a Lipschitz and monotone increasing function. In terms of $\Omega$, this means that $h(\Omega) \in \operatorname{Lip}(\mathbb{R})$ and $(h(\Omega))^{\prime} \geq-\pi \sigma$. We note that an arbitrary growth estimate for $h(\Omega)$ (i.e., the inequality $(h(\Omega))^{\prime} \leq C$ for arbitrary $C$ ) suits us, but the decay estimate is linked with $\sigma:(h(\Omega))^{\prime} \geq-\pi \sigma$. However, in what follows this subtlety will play no role: it is the cruder estimate $\left\|(h(\Omega))^{\prime}\right\|_{L^{\infty}} \leq \pi \sigma$ that will work.

1.12. We summarize the above as follows.

If $0<\omega \leq 1, \mathcal{L}(\omega)>-\infty, h(\Omega) \in \operatorname{Lip}_{1}(\mathbb{R})$, and $\left\|(h(\Omega))^{\prime}\right\|_{L^{\infty}} \leq \pi \sigma$, then the function $m=e^{-h(s)}$ with $s=\pi \sigma x+h(\Omega)-\pi\left[\sigma x+\frac{h(\Omega)}{\pi}\right]-\frac{\pi}{2}$ satisfies the inequality

$$
\log m(x) \leq 4 \sigma+\frac{1}{2}+\frac{\pi}{2} B \log (|x|+2)
$$

and, moreover, $m^{2} \omega^{2} e^{2 \pi i \sigma x}$ is an outer function.

We lack the inclusion $m \omega \in L^{2}$ to assert that $f=\operatorname{Ext} m \omega \in H_{\sigma}^{2}$, and we lack the boundedness of $m$ to conclude that $|f| \leq$ const $\omega$ and prove that $\omega$ is $\sigma$-admissible.

1.13. To cope with these (insignificant) obstructions, we apply the above result not to $\omega$ itself, but to a slightly smaller function $\omega_{0}$,

$$
\omega_{0}=\frac{\omega}{\left(x^{2}+k^{2}\right)^{A}},
$$

where $A=\frac{\pi}{4} B+1$, and the number $k>0$ will be chosen shortly. Assume that $\left\|(h(\Omega))^{\prime}\right\|_{L^{\infty}}<\pi \sigma$ (strict inequality!), and put

$$
\Omega_{0}=\log \frac{1}{\omega_{0}}=\Omega+\gamma,
$$


where $\gamma=A \log \left(x^{2}+k^{2}\right)$. It can be shown (see Subsection 3.6.3) that $\widetilde{\gamma} \in C^{1}(\mathbb{R})$ and $\left\|(h(\gamma))^{\prime}\right\|_{L^{\infty}} \leq$ Const $\frac{A}{k}$. Therefore, $h\left(\Omega_{0}\right) \in \operatorname{Lip}(\mathbb{R})$ and $\left\|\left(h\left(\Omega_{0}\right)\right)^{\prime}\right\|_{L^{\infty}} \leq \pi \sigma$ for some sufficiently large $k$. When applied to $\omega_{0}$, the above result yields a function $m_{0} \geq 0$ such that the function $m_{0}^{2} \omega_{0}^{2} e^{2 \pi i \sigma x}$ is outer and

$$
\log m_{0}(x) \leq 4 \sigma+\frac{1}{2}+\frac{\pi}{2} B \log (|x|+2)
$$

for every $x \in \mathbb{R}$. Consequently,

$$
m_{0}(x) \leq \operatorname{Const}(|x|+2)^{\frac{\pi B}{2}}
$$

and

$$
\omega_{0}(x) m_{0}(x) \leq \operatorname{Const}(|x|+2)^{\frac{\pi B}{2}} \omega(x) /\left(x^{2}+k^{2}\right)^{\frac{\pi B}{4}+1}=\frac{\operatorname{Const} \omega(x)}{x^{2}+k^{2}} .
$$

The last expression does not exceed $\frac{\text { Const }}{x^{2}+k^{2}}$ (because $\omega \leq 1$ ), whence $\omega_{0} m_{0} \in L^{2}$; clearly, the same expression also does not exceed Const $\omega(x)$, and we see that $\omega$ is a $\sigma$-admissible majorant.

1.14. We have obtained the following test for $\sigma$-admissibility.

Theorem 1. If $0<\omega \leq 1, \mathcal{L}(\omega)>-\infty, h(\Omega) \in \operatorname{Lip}(\mathbb{R})$, and $\left\|(h(\Omega))^{\prime}\right\|_{L^{\infty}}<\pi \sigma$, then $\omega$ is a $\sigma$-admissible majorant.

A careful analysis of the proof shows that the condition $\left\|(h(\Omega))^{\prime}\right\|_{L^{\infty}}<\pi \sigma$ can be relaxed to $\overline{\lim }_{x \rightarrow \infty}\left|(h(\Omega))^{\prime}(x)\right|<\pi \sigma$. However, we shall not use this observation seriously (by the way, it shows that the conditions $0<\omega \leq 1, \mathcal{L}(\omega)>-\infty, h(\Omega) \in \operatorname{Lip}_{1}(\mathbb{R})$, and $\lim _{x \rightarrow \infty}\left|(h(\Omega))^{\prime}(x)\right|=0$ imply that $\omega$ is fully admissible).

The function $f_{0} \in H_{\sigma}^{2}$ satisfying $\left|f_{0}\right| \leq$ Const $\omega$ has been constructed in the form Ext $\omega_{0} m_{0}$. At first glance, this formula involves two Hilbert transforms (two "quadratures"): one in the calculation of $h\left(s_{0}\right)$ (because $\left.m_{0}=e^{-h\left(s_{0}\right)}\right)$ and the other in the expression Ext $\omega_{0} m_{0}$. However, the latter one can be avoided by showing that $f_{0}=$ $\varepsilon \omega_{0} m_{0} e^{\pi i \sigma x}$, where $\varepsilon$ is a sign-alternating function equal to +1 or -1 consecutively on the intervals $\left(x_{n}, x_{n+1}\right), x_{n}$ being a unique solution of the equation $\pi \sigma x+h(\Omega)(x)=\pi n$, $n \in \mathbb{Z}$. This and related statements about the structure of $m_{0}$ can be found in HMII, Subsection 2.6]. It is easy to check that, far away from the points $x_{n}$, the factor $m_{0}$ (and, with it, the ratio $\left.\frac{\left|f_{0}\right|}{\omega}\right)$ can be estimated not only from above, but also from below.

1.15. Some applications. Theorem 1 has an unfortunate drawback: its assumptions are imposed on $\widetilde{\Omega}$ rather than directly on $\Omega$, and this hampers their verification and blocks the way to Theorem BM1. However, Theorem 1 would imply that $\omega$ is $\sigma$-admissible if we could find a majorant $\Omega_{1}$ for $\Omega$ such that $\Omega_{1} \in L^{1}(P), \widetilde{\Omega}_{1} \in \operatorname{Lip}_{1}(\mathbb{R})$, and $\left\|\widetilde{\Omega}_{1}^{\prime}\right\|_{\infty}<\pi \sigma$. In this case we would have $\omega_{1} \leq \omega$, where $\omega_{1}=e^{-\Omega_{1}}$ is $\sigma$-admissible (by Theorem 1). In $\S 2$ we shall show that such an $\Omega_{1}$ does exist for every $\sigma>0$ provided $\Omega \in L^{1}(P) \cap \operatorname{Lip}_{1}(\mathbb{R})$, which will finish the proof of Theorem BM1.

But even now we can construct $\Omega_{1} \geq \Omega$ with the required properties if $\Omega$ obeys some additional restrictions. We think that the examples presented below deserve some attention because they are simple and shed more light on the passage to the general setting, which is discussed in $\S 2$. In these examples we replace $\omega$ with its more regular minorant $\omega_{1} \leq \omega$ to which Theorem 1 can be applied directly. It will turn out that $\omega_{1}$ (given by a fairly "explicit" formula) will resemble $\omega$ to a certain extent, which finally will yield some resemblance between the resulting function $|f|$ (with $f \in H_{\sigma}^{2},|f| \leq \omega$ ) and $\omega$ itself at some distance of the zeros of $f$. In the general case (i.e., under the assumptions of Theorem BM1 about full admissibility) we again replace $\omega$ by a more regular minorant. 
But in that setting the passage from $\omega$ to $\omega_{1}$ will be much less "explicit" and, for the most part, no "resemblance" between $\omega_{1}$ and $\omega$ will be possible.

1.16. Here we assume that $\omega=e^{-\Omega}$, where $\Omega>0, \Omega \in L^{1}(P)$, and $\Omega$ is even and monotone increasing on the ray $[0,+\infty)$. This is a classical situation, already touched upon in Subsection 1.5. There is no loss of generality in assuming that $\Omega \equiv 0$ on $\left[-e^{2}, e^{2}\right]$. Put $J(\Omega)=\int_{0}^{\infty}\left(\Omega(x) / x^{2}\right) d x$. Then $J(\Omega)<+\infty$ by assumption. Since $\Omega$ is monotone increasing on $(0,+\infty)$, we see that $\Omega(x)=o(x)$ as $x \rightarrow+\infty$. We introduce the function

$$
r(\Omega)(x)=\int_{0}^{|x|}(\Omega(e t) / t) d t=\int_{e}^{e|x|}(\Omega(u) / u) d u .
$$

It is easily seen that this function is even and monotone increasing on $[0,+\infty), r(\Omega) \equiv 0$ on $[-e, e]$, and $J(r(\Omega))=e J(\Omega)<+\infty$. Furthermore, $r(\Omega)(x) \geq \int_{|x| / e}^{|x|} \Omega(e t) \frac{d t}{t} \geq \Omega(x)$. We take $\Omega_{1}=r(r(\Omega))$. Clearly, $\Omega_{1} \geq r(\Omega) \geq \Omega, J\left(\Omega_{1}\right)=e^{2} J(\Omega)<+\infty$, and $\Omega_{1}(x)=$ $o(x)$ as $x \rightarrow+\infty$. We integrate by parts, taking into account the last estimate and the fact that $\Omega_{1}$ is even, to obtain

$$
h\left(\Omega_{1}\right)(x)=\frac{1}{\pi} \int_{0}^{\infty} \frac{2 x}{x^{2}-t^{2}} \Omega_{1}(t) d t=-\frac{1}{\pi} \int_{0}^{\infty} \log \left|\frac{1+t}{1-t}\right| \frac{r(\Omega)(x e t)}{t} d t
$$

for $x>0$, whence

$$
\widetilde{\Omega}_{1}^{\prime}(x)=-\frac{e}{\pi} \int_{0}^{\infty} \log \left|\frac{1+t}{1-t}\right| \frac{d r(\Omega)(x e t)}{d t} d t=-\frac{1}{\pi} \int_{0}^{\infty} \log \left|\frac{1+t}{1-t}\right| \frac{\Omega\left(e^{2} x t\right)}{x t} d t .
$$

This formula readily implies the relations $h\left(\Omega_{1}\right) \in \operatorname{Lip}_{1}(\mathbb{R})$ and $\widetilde{\Omega}_{1}^{\prime}(x)=o(1)$ as $x \rightarrow+\infty$ (see [HMII, pp. 1292-1293] for more details). The estimate $\omega_{1} \leq \omega$ (where $\omega_{1}=e^{-\Omega_{1}}$ ) and Theorem 1 imply the full admissibility of $\omega$.

For another proof of this result, see Subsection 3.6.9.

1.17. We show that $\omega$ is fully admissible if $\Omega \in \operatorname{Lip}_{\alpha}(\mathbb{R})$ with $0<\alpha<1$ (as usual, it is assumed that $0<\omega \leq 1)$. This is an immediate consequence of the result proved in Subsection 1.16. Indeed, assuming for simplicity that $\Omega \equiv 0$ near the origin, we see that $\Omega(t) \leq \Omega_{1}(t)=K|t|^{\alpha}$ for every $t \in \mathbb{R}$. But the function $\omega_{1}=e^{-\Omega_{1}}$ is fully admissible by Subsection 1.16, and $\omega_{1} \leq \omega$. Even the reference to Subsection 1.16 can be avoided, because a direct calculation yields $h\left(|x|^{\alpha}\right)(t)=c(\alpha) \operatorname{sgn} t \cdot|t|^{\alpha}$, whence

$$
\lim _{|x| \rightarrow \infty} \frac{d h\left(|x|^{\alpha}\right)(t)}{d t}=0,
$$

and $\omega_{1}$ is fully admissible by a direct application of Theorem 1 .

However, this procedure may produce $\omega_{1}$ that deviates greatly from $\omega$, and accordingly, the modulus of the function $f \in H_{\sigma}^{2}$ constructed by using the prescriptions of Subsection 1.14 may happen to be quite dissimilar to $\omega$. (Surely, we do think about the resemblance between $|f|$ and $\omega$, though this theme is absent in the statements; see the discussion of the main problems in Subsection 1.3.) Therefore, we describe another construction of a $\sigma$-admissible minorant $\omega_{1} \leq \omega$ yielding a function $\omega_{1}$ comparable with $\omega$ (i.e., $\omega_{1} \geq c \omega$ ).

We need the following fact: if $0<\alpha<1$ and $F \in \operatorname{Lip}_{\alpha}(\mathbb{R})$, then $h(F) \in \operatorname{Lip}_{\alpha}(\mathbb{R})$. (It should be noted that $\operatorname{Lip}_{\alpha}(\mathbb{R}) \subset L^{1}(P)$ if $0<\alpha<1$.) The proof of this analog of the classical Privalov theorem on conjugate functions (on the circle) can be found (basically) in Ti]: the operator $h_{0}$ instead of $h$ was treated there, but the proof for $h$ is much the same. 
If $\omega=e^{-\Omega}$, where $\Omega \geq 0$ and $\left|\Omega\left(t_{1}\right)-\Omega\left(t_{2}\right)\right| \leq K\left|t_{1}-t_{2}\right|^{\alpha}, t_{1}, t_{2} \in \mathbb{R}$, then by the result cited in the preceding paragraph we have $\left|h(\Omega)\left(t_{1}\right)-h(\Omega)\left(t_{2}\right)\right| \leq K_{1}\left|t_{1}-t_{2}\right|^{\alpha}$, $t_{1}, t_{2} \in \mathbb{R}$. Fixing a large number $L>0$, we put

$$
\Omega^{*}(x)=\frac{1}{2 L} \int_{x-L}^{x+L} \Omega(t) d t, \quad x \in \mathbb{R} .
$$

For every $x \in \mathbb{R}$,

$$
\left|\Omega(x)-\Omega^{*}(x)\right| \leq \frac{1}{2 L} \int_{x-L}^{x+L}|\Omega(t)-\Omega(x)| d t \leq K \cdot(2 L)^{\alpha} .
$$

Thus, $\Omega^{*} \in L^{1}(P)$ and $\Omega \leq \Omega^{*}+K(2 L)^{\alpha}=\Omega_{1}$. Next, $h\left(\Omega_{1}\right)(x)=h\left(\Omega^{*}\right)(x)=$ $\frac{1}{2 L} \int_{x-L}^{x+L} h(\Omega)(t) d t+$ const. Applying the analog of Privalov's theorem quoted above, for fixed $\sigma>0$ and arbitrary $x \in \mathbb{R}$ we obtain

$$
\left|\widetilde{\Omega}_{1}^{\prime}(x)\right|=|h(\Omega)(x+L)-h(\Omega)(x-L)| /(2 L) \leq K_{1} \cdot(2 L)^{\alpha-1}<\pi \sigma
$$

if $L>L(\sigma)$. Now Theorem 1 shows that the function $\omega_{1}=e^{-\Omega_{1}}$ is $\sigma$-admissible (note that $\left.\omega_{1} \geq c(\sigma) \omega\right)$. Since $\omega_{1} \leq \omega$, we see that $\omega$ is $\sigma$-admissible, and it is fully admissible because $\sigma$ is arbitrary.

1.18. By the same method, in HMII the full admissibility of some functions off the union $\bigcup_{0<\alpha<1} \operatorname{Lip}_{\alpha}(\mathbb{R})$ was proved. However, this method falls short of being applicable to arbitrary functions $\Omega \in \operatorname{Lip}_{1}(\mathbb{R}) \cap L^{1}(P)$. For $\alpha=1$, the arguments in Subsection 1.17 do not (and, as we shall see, cannot) furnish a majorant $\Omega_{1} \geq \Omega$ in $L^{1}(P)$ for which the norm $\left\|\widetilde{\Omega}_{1}^{\prime}\right\|_{\infty}$ does not exceed a quantity given beforehand. If $\alpha=1$, the function $K|x|^{\alpha}$ can in no way play the role of such a majorant (it is not integrable with the measure $P)$. Next, for $\alpha=1$ the theorem of Privalov type cited in Subsection 1.17 fails totally. True, from the arguments in Ti] it can be deduced that, at least, $h(\Omega)$ is uniformly continuous if $\Omega$ is a bounded function in $\operatorname{Lip}_{1}(\mathbb{R})$. The last requirement, however, makes $\omega$ bounded away from zero, which denudes the $\sigma$-admissibility problem of any interest. But for unbounded $\Omega \in \operatorname{Lip}_{1}(\mathbb{R}) \cap L^{1}(P)$ (even though of arbitrarily slow growth), $h(\Omega)$ may perfectly fail to be uniformly continuous (if matters are not even worse); an attempt to estimate $\Omega-\Omega^{*}$ uniformly does not meet with success, and the construction outlined in Subsection 1.17 does not work. Precisely this happens in the case of a mountain chain in Figure 3 if the heights and the feet of the mountains are chosen appropriately (that case is of principal interest for us). This is not yet all: a function $\Omega$ depicted in Figure 3 can be chosen in such a way that $\Omega \in \operatorname{Lip}_{1}(\mathbb{R}) \cap L^{1}(P)$, and not only does $h(\Omega)$ fail to be uniformly continuous, but an arbitrary function $\Omega_{1}$ within a finite distance from $\Omega$ in the $L^{\infty}$-metric is "contaminated" by the bad behavior of $\Omega$. More precisely, there is a function $\Omega \geq 0$ in $\operatorname{Lip}_{1}(\mathbb{R}) \cap L^{1}(P)$ such that if $\Omega_{1}$ is measurable and $\left\|\Omega-\Omega_{1}\right\|_{\infty}<+\infty$. Then $h\left(\Omega_{1}\right)$ is not uniformly continuous; see $[\mathrm{BeH}]$. In particular, for such an $\Omega$, no regularization $\Omega * \varphi$ of convolution type, where $\varphi$ has compact support and $\int \varphi=1$, can play the role of $\Omega_{1}$. The required majorant $\Omega_{1}$ of $\Omega$ must jump away from $\Omega$ highly and at many points (i.e., necessarily $\left.\overline{\lim }_{|x| \rightarrow \infty}\left(\Omega_{1}(x)-\Omega(x)\right)=+\infty\right)$. The construction of $\Omega_{1}$ must (and will) differ fundamentally from the construction in Subsection 1.17.

\section{§2. A majorant whose Hilbert transform is Lipschitz}

In what follows, we denote by $\operatorname{Lip}_{1}(k, I)$ the set of all functions defined on a segment $I$ and satisfying the first-order Lipschitz condition with constant not exceeding $k$ :

$$
\left|f\left(x^{\prime}\right)-f\left(x^{\prime \prime}\right)\right| \leq k\left|x^{\prime}-x^{\prime \prime}\right|, \quad x^{\prime}, x^{\prime \prime} \in I .
$$

The symbol $\operatorname{Lip}_{1}(I)$ stands for the union of all classes $\operatorname{Lip}_{1}(k, I), k>0$. 
2.1. The statement of the main result. Our main objective in this section is the following result.

Theorem 2. Let $\Omega$ be a nonnegative function belonging to $L^{1}(P) \cap \operatorname{Lip}_{1}(\mathbb{R})$, and let $\varepsilon>0$. There exists a function $\Omega_{1}$ such that (a) $\Omega_{1} \geq \Omega$; (b) $\Omega_{1} \in L^{1}(P)$; (c) $\Omega_{1} \in \operatorname{Lip}_{1}(\varepsilon, \mathbb{R})$; (d) $h\left(\Omega_{1}\right) \in \operatorname{Lip}_{1}(\varepsilon, \mathbb{R})$.

We remind the reader that $P$ denotes the Poisson measure, $d P(x)=\frac{1}{\pi} \frac{d x}{1+x^{2}}$, and

$$
h(\Omega)(x)=\frac{1}{\pi} \oint_{\mathbb{R}} \Omega(t)\left(\frac{1}{x-t}+\frac{t}{t^{2}+1}\right) d t
$$

(see Subsection 1.2).

As was noted in Subsection 1.18, the function $h(\Omega)$ may fail to be Lipschitz or even uniformly continuous. However, it turns out that some majorant $\Omega_{1}$ of $\Omega$ is better: its Hilbert transform is Lipschitz, and moreover, the two Lipschitz constants $\left\|\Omega_{1}^{\prime}\right\|_{\infty}$ and $\left\|\widetilde{\Omega}_{1}^{\prime}\right\|_{\infty}$ can be made as small as we wish. Precisely such a majorant of $\Omega$ was mentioned in the title of this section.

Combined with Theorem 1 (Subsection 1.14), the statement in this subsection finishes our proof of Theorem BM1.

By a different method, Koosis constructed a function $\Omega_{1}$ that satisfies a)-c) but not d); see [Koo8, p. 80].

2.2. We indicate some notation to be used quite often. The symbol $|I|$ stands for the length of an interval $I, c_{I}$ denotes the center of $I$, and $Q I(Q>0)$ is the interval of length $Q|I|$ centered at $c_{I}$.

We always denote by $A, A_{1}, A_{2}, \ldots$ positive universal constants. The letters $\delta$ and $\sigma$ denote small positive numbers that will occur in intermediate estimates for the norms $\left\|\Omega_{1}^{\prime}\right\|_{\infty}$ and $\left\|\widetilde{\Omega}_{1}^{\prime}\right\|_{\infty}$; at the end of the proof these norms will be made smaller than $\varepsilon$ at the expense of the choice of $\delta$ and $\sigma$.

2.3. The following remark will be needed shortly in the proof of Theorem 2 .

Suppose $\Omega$ satisfies the assumptions of the theorem: $\Omega \in L^{1}(P) \cap \operatorname{Lip}_{1}(k, \mathbb{R}), \Omega \geq 0$. Then $\Omega(x)=o(|x|)$ as $|x| \rightarrow+\infty$. More precisely, for $x>0$ we have

$$
\Omega(x) / x \leq 5 \sqrt{k}\left(\int_{x}^{+\infty} \Omega(t) / t^{2} d t\right)^{1 / 2} ;
$$

for $x<0$ the integral $\int_{x}^{+\infty}$ is replaced by $\int_{-\infty}^{x}$.

Proof. If $x>0$, then $\Omega(x) \leq \Omega(0)+k x$, so that $\Omega(x) / x<2 k$ for $x>\Omega(0) / k$, and under the graph of $\Omega \mid[x, 3 x]$ there is enough room for the triangle depicted in Figure 4.

For such $x$,

$$
\int_{x}^{3 x} \frac{\Omega(t)}{t^{2}} d t \geq \frac{1}{9 x^{2}} \int_{x}^{3 x} \Omega(t) d t \geq \frac{1}{18 k}\left(\frac{\Omega(x)}{x}\right)^{2} .
$$

2.3.1. In the proof of the theorem, it may be assumed without loss of generality that

$$
\Omega \equiv 0 \text { on }[-R, R], \quad \int_{\mathbb{R}} \frac{\Omega(t)}{t^{2}} d t<\sigma, \quad \Omega(x)<\frac{\delta}{2}|x|,
$$

where $\delta, \sigma, R$ are arbitrary positive numbers fixed beforehand ( $\delta, \sigma$ are arbitrarily small, and $R$ is arbitrarily large). The third statement follows from the second and the preceding remark if $\sigma<c(k) \delta^{2}$.

To ensure the first two, we take $B$ so large that

$$
\int_{\{\Omega|t|>B\}}\left(\Omega(t) / t^{2}\right) d t<\sigma \quad \text { and } \quad \Omega<B \text { on }[-R, R] .
$$




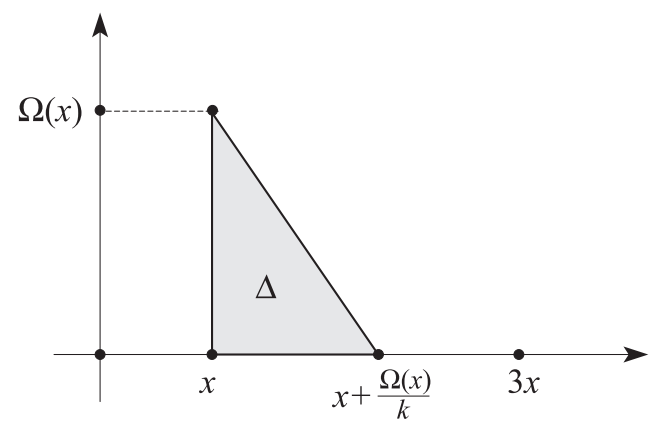

FIGURE 4.

It suffices to prove the theorem for the function $(\Omega-B)^{+}$in place of $\Omega$. Indeed, after finding a function $\Omega_{1, B} \in \operatorname{Lip}_{1}(\varepsilon, \mathbb{R}) \cap L^{1}(P)$ with $\Omega_{1, B} \geq(\Omega-B)^{+}, h\left(\Omega_{1, B}\right) \in \operatorname{Lip}(\varepsilon, \mathbb{R})$, we can put $\Omega_{1}=\Omega_{1, B}+B$ (recall that $h$ (const) $\equiv 0$ ); then $\Omega_{1}$ possesses properties (a)-(d).

2.4. The beginning of the proof and reduction to the quest for a local majorant. We advise the reader to refresh his or her memory about the material of Subsection 1.7 concerning the Hilbert transformation.

2.4.1. Preliminary considerations. Our main concern is in estimating the derivative $\widetilde{\Omega}_{1}^{\prime}$ of the future majorant $\Omega_{1}$ for $\Omega$ (property (d) in the statement of Theorem 2). When differentiating the integral $h\left(\Omega_{1}\right)$ at a point $x$, it is natural to isolate the inconvenient part of this integral corresponding to a neighborhood $V$ of $x$ :

$$
h\left(\Omega_{1}\right)=h\left(\chi_{V} \Omega_{1}\right)+h\left(\chi_{\mathbb{R} \backslash V} \Omega_{1}\right)=\varkappa_{1}+\varkappa_{2}
$$

$\left(\chi_{E}\right.$ denotes the characteristic function of a set $E$ ). When calculating the derivative $\varkappa_{2}^{\prime}(x)$, we can differentiate under the integral sign; next, $(x-t)^{2} \asymp t^{2}$ for $t \notin V$ if $V=\{t:|x-t|<|x| / 2\}$, so we have

$$
\left|\varkappa_{2}^{\prime}(x)\right| \leq A \int_{\mathbb{R} \backslash V} \frac{\Omega_{1}(t)}{(x-t)^{2}} d t \leq A_{1} \int_{\mathbb{R}} \frac{\Omega_{1}(t)}{t^{2}} d t .
$$

We saw in Subsection 1.18 that, generally speaking, for many values of $x$ the quantity $\Omega_{1}(x)$ turns out to be much greater than $\Omega(x)$. But this does not forbid the estimate

$$
\int_{\mathbb{R}} \frac{\Omega_{1}(t)}{t^{2}} d t \leq C \int_{\mathbb{R}} \frac{\Omega(t)}{t^{2}} d t
$$

This estimate will really be ensured (with $C=C\left(\left\|\Omega^{\prime}\right\|_{\infty}, \delta\right)$ ), and then (2.1) and (2.2) will yield

$$
\left|\varkappa_{2}(x)\right| \leq A_{1} C \sigma
$$

for all $x$.

Estimate (2.3) is the only condition expressing the nearness (quite weak) of $\Omega_{1}$ to $\Omega$.

2.4.2. The above considerations suggest the following procedure. Put

$$
I_{j}=\left[2^{j}, 2^{j+1}\right], \quad I_{-j}=-I_{j}, \quad j=1,2, \ldots ; \quad I_{0}=[-2,2] .
$$

We assume (see Subsection 2.3.1) that $\Omega \equiv 0$ on $[-2,2]$ and that

$$
\int_{\mathbb{R}}\left(\Omega(t) / t^{2}\right) d t<\sigma, \quad \Omega(x) /|x|<\delta / 2 \quad \text { for all } x \in \mathbb{R} \backslash\{0\} .
$$


We shall construct majorants $\Omega_{1, j}$ for every piece $\Omega_{j}=\Omega \mid I_{j}(j \in \mathbb{Z})$ of $\Omega$ separately. Imagine that the piece $\Omega_{j}$ is covered with a $C^{\infty}$-function $\Omega_{1, j} \geq 0$ vanishing off $3 / 2 I_{j}$, i.e., $\Omega \leq \Omega_{1, j}$ on $I_{j}$. We put $\Omega_{1,0} \equiv 0$ and

$$
\Omega_{1}=\sum_{j \in \mathbb{Z}} \Omega_{1, j} .
$$

Since the covering $\left\{2 I_{j}\right\}$ of $\mathbb{R}$ has finite multiplicity, we obtain

$$
\Omega \leq \Omega_{1}, \quad \Omega_{1} \in C^{\infty}(\mathbb{R}), \quad \Omega_{1} \equiv 0 \text { on }[-1,1] .
$$

Next, on every segment $2 I_{j}$ with $j \neq 0$ we have

$$
2^{-2|j|-2} d x \leq d x / x^{2} \leq 2^{-2|j|} d x .
$$

If we manage to ensure the inequality

$$
\int_{3 / 2 I_{j}} \Omega_{1, j}(x) d x \leq C \int_{I_{j}} \Omega_{j}=C \int_{I_{j}} \Omega
$$

for all $j \in \mathbb{Z}$ with $C=C\left(\left\|\Omega^{\prime}\right\|_{\infty}, \delta\right)$, then, clearly,

$$
\begin{aligned}
\int_{\mathbb{R}} \frac{\Omega_{1}(x)}{x^{2}} d x & =\sum_{j \neq 0} \int_{2 I_{j}} \frac{\Omega_{1, j}(x)}{x^{2}} d x \leq C \sum_{j \neq 0}\left(\int_{I_{j}} \Omega(x) d x\right) 2^{-2|j|} \\
& \leq 4 C \sum_{j \neq 0} \int_{I_{j}} \frac{\Omega(x)}{x^{2}} d x=4 C \int_{\mathbb{R}} \frac{\Omega(x)}{x^{2}} d x<+\infty
\end{aligned}
$$

by (2.5), so that $\Omega_{1} \in L^{1}(P)$, and $h\left(\Omega_{1}\right)$ makes sense. Thus, inequalities (2.5) (conjectural for the moment) ensure property (b) of the majorant $\Omega_{1}$. We turn to property (c) and, chiefly, to (d). Fix $x \in \mathbb{R}$ and take $j(x) \in \mathbb{Z}$ with $x \in I_{j(x)}$. Let $I_{j_{+}(x)}$ and $I_{j_{-}(x)}$ be the right and the left neighbor of $I_{j(x)}$ in the sequence $\left\{I_{j}\right\}_{j \in \mathbb{Z}}$. Then

$$
\Omega_{1}(t)=\Omega_{1, j_{-}(x)}(t)+\Omega_{1, j(x)}(t)+\Omega_{1, j_{+}(x)}(t)+\sum_{x} \Omega_{1, j}(t), \quad t \in \mathbb{R},
$$

where $\sum_{x}=\sum_{j \neq j(x), j_{ \pm}(x)}$. If $j \neq j(x), j_{ \pm}(x)$, and $t \in I_{j(x)}$, then

$$
\begin{aligned}
\left|h\left(\Omega_{1, j}\right)(t)\right| & =\frac{1}{\pi}\left|\int_{3 / 2 I_{j}} \Omega_{1, j}(s) \frac{s t+1}{(t-s)\left(s^{2}+1\right)} d s\right| \\
& \leq A_{1}|t| \int_{3 / 2 I_{j}} \frac{\Omega_{1, j}(s)}{s^{2}} d s \leq A_{2} C|t| \int_{I_{j}} \frac{\Omega(s)}{s^{2}} d s ;
\end{aligned}
$$

the last inequality follows from (2.5). These estimates show that

$$
h\left(\Omega_{1}\right)(t)=h\left(\Omega_{1, j_{-}(x)}\right)(t)+h\left(\Omega_{1, j(x)}\right)(t)+h\left(\Omega_{1, j_{+}(x)}\right)(t)+\sum_{x} h\left(\Omega_{1, j}\right)(t)
$$

for $t \in I_{j(x)}$. The first three summands and the last sum are infinitely differentiable on $I_{j(x)}$, whence $h\left(\Omega_{1}\right) \in C^{\infty}(\mathbb{R})$.

In Subsection 2.4.1 it was shown that for $t \in I_{j(x)}$ it is possible to differentiate termwise in (2.8) (now $I_{j_{-}(x)} \cup I_{j(x)} \cup I_{j_{+}(x)}$ plays the role of $V$ ), and we obtain

$$
\widetilde{\Omega}_{1}^{\prime}(x)=\widetilde{\Omega}_{1, j_{-}(x)}^{\prime}(x)+\widetilde{\Omega}_{1, j(x)}^{\prime}(x)+\widetilde{\Omega}_{1, j_{+}(x)}^{\prime}(x)+\sum_{x} \widetilde{\Omega}_{1, j}^{\prime}(x),
$$

because the series $\sum_{x} \widetilde{\Omega}_{1, j}^{\prime}(t)$ converges uniformly in $t \in I_{j(x)}$. Consequently, if estimates (2.5) are true, then

$$
\left|\widetilde{\Omega}_{1}^{\prime}(x)\right| \leq\left|\widetilde{\Omega}_{1, j_{-}(x)}^{\prime}(x)\right|+\left|\widetilde{\Omega}_{1, j(x)}^{\prime}(x)\right|+\left|\widetilde{\Omega}_{1, j_{+}(x)}^{\prime}(x)\right|+A_{3} C \sigma, \quad C=C\left(\left\|\Omega^{\prime}\right\|_{\infty}, \delta\right)
$$


(see (2.3) and (2.1)). If we could ensure the inequalities

$$
\left\|\widetilde{\Omega}_{1, j_{ \pm}(x)}^{\prime}\right\|_{\infty} \leq A \delta, \quad\left\|\widetilde{\Omega}_{1, j(x)}^{\prime}\right\|_{\infty} \leq A \delta,
$$

we would finally obtain the estimate

$$
\left|\widetilde{\Omega}_{1}^{\prime}(x)\right| \leq 3 A \delta+C\left(\left\|\Omega^{\prime}\right\|_{\infty}, \delta\right) \sigma
$$

for every $x \in \mathbb{R}$. Choosing $\delta<\varepsilon /(6 A)$ and then a small $\sigma>0$, we would arrive at the principal estimate, i.e., statement (d) of the theorem: $\left\|\widetilde{\Omega}_{1}^{\prime}\right\|_{\infty}<\varepsilon$. As to statement (c) (frankly speaking, included merely because of greed: it is not required for the proof of Theorem BM1), it would be a consequence of the inequalities

$$
\left\|\Omega_{1, j}^{\prime}\right\|_{\infty} \leq A \delta
$$

and the fact that the covering $\left\{2 I_{j}\right\}$ has finite multiplicity. We summarize: a global (i.e., defined on the entire set $\mathbb{R}$ ) majorant $\Omega_{1} \geq \Omega$ with the required properties will be constructed if we manage to find nonnegative local majorants $\Omega_{1, j} \geq \Omega_{j}=\Omega \mid I_{j}$ of class $C^{\infty}(\mathbb{R})$ that are supported on $3 / 2 I_{j}$ and possess properties $(2.5),(2.9)$, and (2.10).

2.4.3. The statement of the local majorization problem. We have reduced the proof of Theorem 2 to the following problem of local majorization (i.e., majorization on a bounded interval).

Given $\delta>0, k>0$, a bounded closed interval $I$, and a nonnegative function $f \in$ $\operatorname{Lip}_{1}(k, I)$, find a nonnegative function $F \in C^{\infty}(\mathbb{R})$ with the following properties:

1) $F \equiv 0$ off $3 / 2 I$

2) $F \geq f$ on $I$

3) $\left\|F^{\prime}\right\|_{\infty} \leq A \delta$;

4) $\left\|\widetilde{F}^{\prime}\right\|_{\infty} \leq A \delta$

5) $\int_{\mathbb{R}} F \leq C \int_{I} f$, where $C$ may depend on $k$ and $\delta$, but not on $I$.

Put $I=I_{j}$ and $f=\Omega_{j}=\Omega \mid I_{j}$. If our problem is solvable for such $f$ and $I$, then we can take the function $F$ for the role of a local majorant $\Omega_{1, j}$ possessing properties (2.5), (2.9), and (2.10). This will finish the proof of Theorem 2 in Subsection 2.1 and, with it, of Theorem BM1.

2.5. Local problem: preliminary considerations. First, we observe the invariance of the problem under certain transformations of the function and the independent variable.

2.5.1. We reserve the notation $I^{*}$ for the interval $[-1 / 2,1 / 2]$. Let $g$ be a function defined at least on $I^{*}$, let $I$ be some closed interval, and let $c_{I}$ be its center. We put

$$
g_{I}(x)=|I| g\left(\frac{x-c_{I}}{|I|}\right) .
$$

The function $g_{I}$ is a sort of a homothetic copy of $g$ adjusted to $I$. If $g$ is supported on $Q I^{*}$, where $Q>1$, then $g_{I}$ is supported on $Q I$. If $g \in \operatorname{Lip}_{1}\left(I^{*}\right)$, then $g_{I} \in \operatorname{Lip}_{1}(I)$ and $\left\|g_{I}^{\prime}\right\|_{\infty}=\left\|g^{\prime}\right\|_{\infty}$. Next, if $g \in C^{\infty}(\mathbb{R})$ and $g \equiv 0$ off $Q I^{*}$, then $h_{0}\left(g_{I}\right)=\left(h_{0}(g)\right)_{I}$ and $\left\|\tilde{g}_{I}^{\prime}\right\|_{\infty}=\left\|\tilde{g}^{\prime}\right\|_{\infty}$. Finally,

$$
\int_{I} g_{I}=|I|^{2} \int_{I^{*}} g .
$$

These remarks show that it suffices to solve the local majorization problem (see Subsection 2.4.3) for the standard interval $I^{*}=[-1 / 2,1 / 2]$. Indeed, given a nonnegative function $f \in \operatorname{Lip}_{1}(k, I)$, we find a function $f^{*} \in \operatorname{Lip}_{1}\left(k, I^{*}\right)$ with $f=f_{I}^{*}$. If there exists a nonnegative function $F^{*} \in C^{\infty}(\mathbb{R})$ such that $F^{*} \equiv 0$ off $3 / 2 I^{*}=[-3 / 4,3 / 4], F^{*} \geq f^{*}$ on $I^{*},\left\|F^{* \prime}\right\|_{\infty} \leq A \delta,\left\|\widetilde{F}^{* \prime}\right\|_{\infty} \leq A \delta$, and $\int_{I^{*}} f^{*} \geq C \int_{\mathbb{R}} F^{*}$, then $F=F_{I}^{*}$ will be a solution of our problem for the initial $f$ and $I$. 


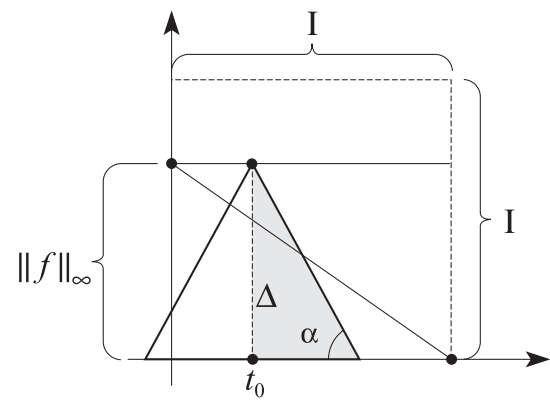

FIGURE 5 .

2.5.2. Now some discussion of our problem is in order (in the initial wording of Subsection 2.4.3, i.e., for arbitrary $f$ and $I$ ). Temporarily, we put $\delta=1$ in conditions 3 ) and 4 ); then $C$ in 5) depends entirely on $k$.

Here we need a standard smooth "cap" $\varphi^{*}$, which we choose and fix once and for all:

$$
\varphi^{*} \in C^{\infty}(\mathbb{R}), \quad 0 \leq \varphi^{*} \leq 1, \quad \varphi^{*} \equiv 0 \text { off } 3 / 2 I^{*}, \quad \varphi^{*} \equiv 1 \text { on } I^{*} .
$$

We return to our problem. Conditions 1) and 2) of it can be satisfied quite easily by taking $F=\|f\|_{\infty} \chi_{I}$. It is necessary to regularize this step function, which forces us to take

$$
F(x)=\|f\|_{\infty}|I|^{-1} \varphi_{I}^{*}(x)=\|f\|_{\infty} \varphi^{*}\left(\frac{x-c_{I}}{|I|}\right), \quad x \in \mathbb{R},
$$

so that $F \geq 0, F \in C^{\infty}(\mathbb{R}), F \geq f$ on $I, F$ vanishes off $3 / 2 I$. Next, the obvious identities

$$
\left\|F^{\prime}\right\|_{\infty}=\|f\|_{\infty}|I|^{-1}\left\|\varphi^{* \prime}\right\|_{\infty}, \quad\left\|\widetilde{F}^{\prime}\right\|_{\infty}=\|f\|_{\infty}|I|^{-1}\left\|\widetilde{\varphi}^{* \prime}\right\|_{\infty}
$$

cause us to subject $f$ to the condition

$$
\|f\|_{\infty} \leq|I|,
$$

in order to ensure the estimates $\left\|F^{\prime}\right\|_{\infty} \leq A$ and $\left\|\widetilde{F}^{\prime}\right\|_{\infty} \leq A$ that coincide with 3) and 4) for $\delta=1$; here $A=\max \left(\left\|\varphi^{* \prime}\right\|_{\infty},\left\|\widetilde{\varphi}^{* \prime}\right\|_{\infty}\right)$. Condition (2.11) means that the graph of $f$ is included in the square $I \times[0,|I|]=S(I)$.

2.5.3. We turn to the most problematic condition 5):

$$
\int_{I} f \geq C \int_{I} F\left(\geq C\|f\|_{\infty}|I|\right),
$$

where $C=C(k)$. If we disregard the assumption $f \in \operatorname{Lip}_{1}(k, I)$, then (2.12) seems to be absolutely unbelievable, because, generally speaking, $\int_{I} f$ may be very much smaller than $\|f\|_{\infty}|I|$. But since $f$ is Lipschitz (moreover, $\left\|f^{\prime}\right\|_{\infty} \leq k$ ), some hope for (2.12) remains nonetheless, provided $\|f\|_{\infty}$ is relatively large compared to $|I|$.

2.5.4. Digression: a lower estimate for the integral of a Lipschitz function. Let $t_{0} \in I$, and let $f\left(t_{0}\right)=\|f\|_{\infty}$. Suppose that

$$
k \geq \frac{\|f\|_{\infty}}{|I|} .
$$

A glance at Figure 5, where $\tan \alpha=k$, shows us that either the left or the right half of the triangle $\Delta$ is included in the subgraph of $f$. The legs of this half are $\|f\|_{\infty}$ and $\|f\|_{\infty} / k$, whence $\int_{I} f \geq\|f\|_{\infty}^{2}(2 k)^{-1}$. 
In what follows, we assume without loss of generality that $k \geq 1$. Then (2.13) follows from (2.11). Thus, if $k \geq 1$ and $\|f\|_{\infty} \leq|I|$, then

$$
2 k \int_{I} f \geq\|f\|_{\infty}^{2}
$$

This estimate is sharp, which can again be seen from Figure 5 (it suffices to take $f$ for which $t_{0}$ is the left end of $I, f^{\prime} \equiv-k$ on $\left(t_{0}, t_{0}+\|f\|_{\infty} / k\right)$, and $f \equiv 0$ on the remaining part of $I)$. Therefore, in order to ensure (2.12), i.e., to meet condition 5$)$, we need to assume that $\|f\|_{\infty}^{2} \geq A\|f\|_{\infty}|I|$, where $A \in(0,1)$.

2.5.5. Taking $A=1 / 2$, we arrive at the following conclusion: if $k \geq 1$ and

$$
1 / 2 \leq\|f\|_{\infty} /|I| \leq 1,
$$

then the local majorization problem (with $\delta=1$ ) is solvable, and the cap $\varphi_{I}^{*}$ may play the role of the majorant $F$.

This definition of $F$ differs inessentially from the initial one (i.e., from $\|f\|_{\infty}|I|^{-1} \varphi_{I}^{*}$ ); see (2.15). Estimate (2.15) means that the graph of $f$ is included in the square $S(I)$ and intersects its upper half (or the line passing through the midpoints of the lateral edges). In this case, by (2.14) and the inequality $\|F\|_{\infty} \leq|I|$, we have

$$
\int_{I} f \geq \frac{1}{8 k}|I|^{2} \geq \frac{1}{8 k} \cdot \frac{1}{2} \int_{3 / 2 I} F=\frac{1}{16 k} \int_{\mathbb{R}} F,
$$

so that 5$)$ is fulfilled with $C=(16 k)^{-1}$.

See a remark in Subsection 3.6.7 about the relationship between estimate (2.14) and the Hadamard-Landau inequality for derivatives.

2.5.6. The case of an arbitrary positive $\delta$. This case is easily reduced to the case of $\delta=1$. Specifically, if

$$
\delta / 2 \cdot|I| \leq\|f\|_{\infty} \leq \delta|I|,
$$

then $f / \delta$ (in place of $f$ ) satisfies all assumptions of the problem and condition (2.15) (but $k$ should be replaced with $k / \delta$, and we should suppose that $k \geq \delta$ ). In accordance with Subsection 2.5.5, we find a majorant $F_{\delta}$ for $f / \delta$ such that $F_{\delta} \in C^{\infty}(\mathbb{R}), F_{\delta} \geq 0$, $F_{\delta} \equiv 0$ off $3 / 2 I,\left\|F_{\delta}^{\prime}\right\|_{\infty},\left\|\widetilde{F}_{\delta}^{\prime}\right\|_{\infty} \leq A$, and $\delta^{-1} \int_{I} f \geq(\delta / 16 k) \int_{\mathbb{R}} F_{\delta}$. Clearly, $F=\delta F_{\delta}$ satisfies all requirements of the problem $(C=\delta / 16 k$ in condition 5$))$.

2.5.7. Some conclusions. Thus, the condition

$$
\delta|I| / 2 \leq\|f\|_{\infty} \leq \delta|I|
$$

suffices for the solvability of the local majorization problem. The second inequality (but not the first!) is fulfilled automatically in the case that is most important for us, where $I=I_{j}$ and $f=\Omega_{j}=\Omega \mid I_{j}$, - see the end of Subsection 2.4.2. But the first inequality may well happen to be violated. Nevertheless, we shall show that, despite all, the required majorant $F=\Omega_{1, j}$ exists.

If $\|f\|_{\infty}<(\delta / 2)|I|$, it is natural to resort to splitting $I$ into two halves $I_{ \pm}$in the hope that for them the inequalities $\|f\|_{\infty, \pm} \geq(\delta / 2)\left|I_{ \pm}\right|=(\delta / 4)|I|$ will be true (the upper estimates $\|f\|_{\infty, \pm} \leq \delta\left|I_{ \pm}\right|$are true by assumption). Then we find the required majorants $F_{ \pm}$for $f$ (more precisely, for $f_{ \pm} \mid I_{ \pm}$), and $F_{+}+F_{-}$will majorize $f$ on $I$. If one of the norms $\left\|f_{ \pm}\right\|_{\infty}$ (or both) turns out to be smaller than $\delta\left|I_{+}\right| / 2$, then we continue splitting into two halves and stop this procedure whenever a "good" interval occurs. In the proof of the main lemma, we shall act in this spirit (see Subsections 2.6 and 2.7). 
2.6. Main lemma (the statement). Suppose $I$ is a closed bounded interval, $\delta \in(0,1)$, $k \geq 1, f \in \operatorname{Lip}_{1}(k, I)$, and

$$
f \geq 0, \quad\|f\|_{\infty} \leq \delta|I|
$$

Then there exists a function $F \in C^{\infty}(\mathbb{R}), F \geq 0$, with

1) $F \equiv 0$ off $(3 / 2)|I|$,

2) $F \geq f$ on $I$,

3) $\left\|F^{\prime}\right\|_{\infty} \leq A \delta$,

4) $\left\|\widetilde{F}^{\prime}\right\|_{\infty} \leq A \delta$,

5) $\int_{I} f \geq \frac{A_{1} \delta}{k} \int_{\mathbb{R}} F$.

Recall once again that if (2.18) is accompanied by the lower estimate $\|f\|_{\infty} \geq(\delta / 2)|I|$, then for the required majorant $F$ we can take the cap $\delta \varphi_{I}^{*}$ (see Subsection 2.5.6).

It suffices to prove the main lemma for $I=I^{*}=[-1 / 2,1 / 2]$ and $\delta=1$ (see Subsections 2.5.1 and 2.5.6). Only this particular case will be treated in Subsections 2.6 and 2.7 .

2.6.1. Dyadic cells: a reminder. The process of consecutive splitting into halves indicated above leads to the so-called dyadic intervals. Whereas on the way towards the statement of the main lemma we worked with $b i g$ dyadic pieces $\Omega_{j}$ of $\Omega$ (i.e., with restrictions of $\Omega$ to big segments $I_{j}= \pm\left[2^{|j|}, 2^{|j|+1}\right], j \in \mathbb{Z}$, running away to infinity), in the proof of that lemma we shall need small dyadic pieces of $f$ (in fact, small pieces of $\Omega_{j}$ with $j$ fixed). In other words, we concentrate on the restrictions of $f$ to the intervals of the form $\left[m \cdot 2^{-l},(m+1) \cdot 2^{-l}\right) \subset I^{*}=[-1 / 2,1 / 2], l \in \mathbb{Z}_{+}$.

Instead of closed dyadic segments, we shall work with half-open intervals $\left[m \cdot 2^{-l}\right.$, $\left.(m+1) \cdot 2^{-l}\right)$, to be called cells (for short, we omit the adjective "dyadic").

The number $l$ is called the rank of a cell. We only need cells of positive rank, and, as an exception, one cell of rank 0 , i.e., $[-1 / 2,1 / 2)$.

For two arbitrary cells, either they do not intersect, or one of them includes the other. Therefore, if $A^{\text {max }}$ denotes the set of maximal elements (by inclusion) for an arbitrary set $A$ of cells, then

$$
\bigcup_{a \in A} a=\bigcup_{a \in A^{\max }} a
$$

moreover, the cells in $A^{\max }$ are pairwise disjoint.

The passage from $A$ to $A^{\max }$ is in fact fairly constructive: at the first step we form the set $A_{l_{1}} \subset A$ of all cells in $A$ of the highest rank $l_{1}$, then form the set $A_{l_{2}} \subset A$ of all cells of maximal rank $l_{2}$ among those belonging to $A$ but not included in any cell in $A_{l_{1}}$, and so on. It is easily seen that

$$
A^{\max }=\bigcup_{j=1}^{N} A_{l_{j}}, \quad N \leq+\infty .
$$

The transformation $A \mapsto A^{\max }$ will be applied at decisive steps of the proof of the main lemma. Maybe, this procedure of constructing $A^{\max }$ becomes more pictorial if, with every cell $a$, we link the square $S(a)=a \times[0,|a|)$. Then the transformation $A \mapsto A^{\max }$ can be perceived as a "downward movement with stops" in the principal square $S([-1 / 2,1 / 2))$. Of two squares forming its lower half, we choose that (those) with base in $A$; then we pass to the lower half (halves) of the remaining square(s), and among two squares forming such a half we choose that (those) with base in $A$, and so on. The bases of all squares selected at some step form precisely the set $A^{\max }$. 


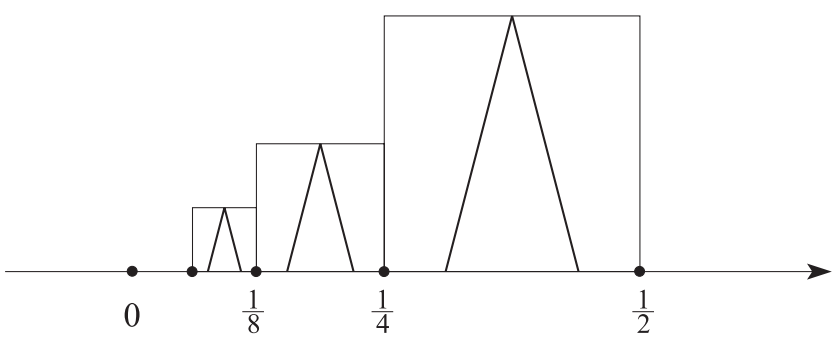

FiguRE 6.

2.6.2. Proof of the main lemma (the beginning). We agree to say that an interval $a \subset I^{*}$ is long (" $f$-long" would be a more precise term) if $\left\|f\left|a \|_{\infty} \leq\right| a \mid\right.$. Note that $I^{*}$ is long by assumption. If $\left\|f\left|a \|_{\infty} \geq\right| a \mid / 2\right.$, the interval $a$ is said to be essential. If an interval $a$ is both long and essential and $k \geq 1$ (which is assumed throughout), then

$$
\int_{a} f \geq \frac{|S(a)|}{8 k}
$$

where $|\cdot|$ denotes area. Furthermore, in this case $\varphi_{a}^{*}$ satisfies conditions 1)-5) of the main lemma relative to the piece $f \mid a$ of $f$ (i.e., when $I=a$ and $f$ is replaced with $f \mid a$; the role of $F$ is played by $\varphi_{a}^{*}$; see the end of Subsection 2.5.4). Next, $\int_{a} \varphi_{a}^{*} \geq(16 k)^{-1}|S(a)|$.

Suppose that the interval $I^{*}$ (or at least the set $\{f>0\}$ ) is covered by a system $\tau$ of pairwise disjoint long intervals, and that, moreover,

$$
\text { the intervals } 2 a, a \in \tau \text {, cover the set } \bigcup_{a \in \tau} 2 a
$$

with multiplicity at most $A_{1}$.

Put $F_{\tau}=\sum_{a \in \tau} \varphi_{a}^{*}$. Then $F_{\tau} \geq f$ on $I^{*}, F_{\tau} \geq 0, F_{\tau} \in C^{\infty}(\mathbb{R}), F_{\tau} \equiv 0$ off $3 / 2 I^{*}$, and $\left\|F_{\tau}^{\prime}\right\|_{\infty} \leq A_{1}\left\|\varphi^{* \prime}\right\|_{\infty}=A_{2}$ (for the moment, we postpone estimation of $\left\|\widetilde{F}_{\tau}^{\prime}\right\|_{\infty}$ ). If, moreover, all intervals $a \in \tau$ are essential, then

$$
\int_{\mathbb{R}} F_{\tau}=\sum_{a \in \tau} \int_{2 a} \varphi_{a}^{*} \leq 2 \sum_{a \in \tau}|a|^{2} \leq 16 k \sum_{a \in \tau} \int_{a} f=16 k \int_{I^{*}} f .
$$

So, if all intervals in $\tau$ are both long and essential, then, under condition $\left(2.18^{\prime}\right)$, the function $F$ could serve as a majorant promised in the main lemma, provided that we would be able to exhibit a universal upper estimate for $\left\|\widetilde{F}^{\prime}\right\|_{\infty}$.

2.6.3. However, here we encounter an obstruction illustrated by the function $f_{N}$ in Figure 6.

For this function, all intervals $a_{j}=\left[2^{-(j+1)}, 2^{-j}\right), j=1, \ldots, N$, are long and essential. Together with $\left[-1 / 2,1 / 2^{N+1}\right]$, they form a partition $\tau_{N}$ of $[-1 / 2,1 / 2)$; condition $\left(2.18^{\prime}\right)$ is fulfilled with $A_{1}=4$. Now we observe that if $a$ is an arbitrary bounded interval, $x \notin 3 /(2 a)$, and $\operatorname{dist}(x, 2 a) \leq 2|a|$, then

$$
\widetilde{\varphi}_{a}^{* \prime}(x)=-\frac{1}{\pi} \int \frac{\varphi_{a}^{*}(t)}{(x-t)^{2}} d t \leq-\frac{1}{4 \pi|a|^{2}} \int_{2 a} \varphi_{a}^{*} \leq-\frac{1}{4 \pi}
$$

(recall that $\varphi_{a}^{*} \equiv|a|$ on $a$ ). Therefore (see Figure 6),

$$
\widetilde{F}_{\tau_{N}}^{\prime}(0)=\sum_{j=1}^{N} \widetilde{\varphi}_{a_{j}}^{* \prime}(0) \leq-\frac{N}{4 \pi}, \quad\left\|\widetilde{F}_{\tau_{N}}^{\prime}\right\|_{\infty} \geq \frac{N}{4 \pi} .
$$

The obstruction comes from the fact that, near the point $x=0$, a buildup of intervals $a$ with $d(x, 2 a) \asymp|a|$ occurs. To struggle with dangerous accumulations of this sort, we 
must "smoothen" the system $\tau$ by replacing it with a "regular" system $r$ in which the lengths of intervals accumulating to a point decay more slowly. Looking at Figure 6 once again, note that $\tau_{N}$ can be replaced by the triple $r=\{[-1,0],[0,1 / 4],[1 / 4,1 / 2]\}$, and $F_{\tau_{N}}$ by the sum $F_{\tau}$ of only three caps, which leads to a new majorant $F_{\tau}$ of all functions $f_{N}$ at once with the estimate $\left\|\widetilde{F}_{\tau}^{\prime}\right\|_{\infty} \leq 3\left\|\widetilde{\varphi}^{* \prime}\right\|_{\infty}$.

2.6.4. Returning to the function $f$ in the main lemma, consider the set $A_{f}$ of all essential cells (we recall that a "cell" always means a "dyadic cell"). Clearly,

$$
\{x \in[-1 / 2,1 / 2): f(x)>0\}=\bigcup_{a \in A_{f}} a
$$

(indeed, if $-1 / 2 \leq x<1 / 2$ and $f(x)>0$, then $f(x)>2^{-l}$ for some $l>1$, and $x$ is contained in some cell $a \subset I^{*}$ of rank $l-1$, i.e., $x \in a$ and $f(x)>\frac{|a|}{2}$, whence $\left.a \in A_{f}\right)$. Now we observe that $A_{f}^{\max }$ consists of long cells: if $|a|=1$, then $a=[-1 / 2,1 / 2)$, and $a$ is long by the assumptions of the lemma; but if $|a|<1$, then $a \subset b \subset[-1 / 2,1 / 2)$, where $b$ is a cell with $|b|=2|a|$, and if $a$ is not long, then $b \in A_{f}$, contradicting the maximality of $a$ in $A_{f}$.

Since $\bigcup_{a \in A_{f}^{\max }} a=\bigcup_{a \in A_{f}} a$, we see that $A_{f}^{\max }$ is a partition of the set $\{x \in[-1 / 2,1 / 2)$ : $f(x)>0\}$ into long essential cells. The function $\sum_{a \in A_{f}^{\max }} \varphi_{a}^{*}$ majorizes $f$ on $I^{*}$, and the integral of this majorant over $\mathbb{R}$ does not exceed the quantity $16 k \int_{I^{*}} f$ (see $(2.19)$ ). Yet we need to control the multiplicity of the system $\left\{2 a: a \in A_{f}^{\max }\right\}$ and to cope with possible obstructions discussed in Subsection 2.6.3. For this, we shall need to replace the system $\tau=A_{f}^{\max }$ with a more regular system $r$ and, instead of $\sum_{a \in \tau} \varphi_{a}$, take the function

$$
F=F_{r}=\sum_{a \in r} \varphi_{a}
$$

for the role of a majorant for $f$.

2.6.5. Regularization of the system $\tau$ : adjoining tails. In order to construct a regular system $r$ of cells, with every cell $a$ we associate its tail $t(a)$, which consists of certain cells not intersecting $a$ and having the property

$$
\sum_{b \in t(a)}|S(b)| \leq 6|S(a)| .
$$

To clarify the target for the subsequent construction, we postpone the explicit description of the command $a \mapsto t(a)$ and the definition of the regular system $r$, but postulate two properties of that system required in the sequel:

(i) $r$ consists of long (not necessarily essential) mutually disjoint cells lying in $I^{*}$ and covering $[-1 / 2,1 / 2)$; moreover, $r=r_{1} \cup r_{2}$, where $r_{1} \subset \tau=A_{f}^{\max }$ and every cell $a \in r_{2}$ belongs to the tail of some cell $b \in \tau$;

(ii) if $a, b \in r$ and $|a|=2^{k}|b|$ with some $k \geq 2$, then $d(a, b) \geq(3 / 2)^{k-1}|a|(d(a, b)$ denotes the distance between $a$ and $b$ ).

Property (ii) expresses the required regularity of $r$ : if its element $a$ is many times as long as its element $b$, then $b$ is very far from $a$ compared to the length of $a$.

2.6.6. A consequence of condition (ii). Denote by $N(a)$ the set of all "neighbors" of $a$ in the system $r$ (i.e., of all cells close to $a$ in length and lying not far from $a$ ):

$$
N(a)=\{b \in r: d(a, b) \leq 2|a|, 1 / 2 \leq|a| /|b| \leq 2\} .
$$

Clearly, \#N(a) $\leq 9$ (at most four "neighbors" may be adjacent to $a$ from each side). 
The required corollary says that if $b \in r \backslash N(a)$, then either $|b| \leq 2|a|$, and in this case $d(2 a, 2 b) \geq|a| / 2$, or $|b|=2^{k}|a|$ for some $k \geq 2$, and in that case

$$
d(2 a, 2 b) \geq 2 \cdot 3^{k-2}|a| .
$$

Proof. Suppose $b \in r \backslash N(a)$. If $1 / 2 \leq|a| /|b| \leq 2$, then $d(a, b)>2|a|$, and the inequality $d(2 a, 2 b) \geq|a| / 2$ follows directly. If $|a|>2|b|$, then $|a| \geq 4|b|$, and by (i) we have

$$
d(2 a, 2 b)=d(a, b)-(|a|+|b|) / 2 \geq d(a, b)-|a| \geq((3 / 2)-1)|a|=|a| / 2 .
$$

Finally, if $|b|>2|a|$, then $|b|=2^{k}|a|$ for some $k \geq 2$, and

$$
\begin{aligned}
d(2 a, 2 b) & =d(a, b)-(|a|+|b|) / 2 \geq d(a, b)-|b| \\
& \geq|b|\left((3 / 2)^{k-1}-1\right) \geq(1 / 3) \cdot(3 / 2)^{k-1}|b| \\
& =(1 / 3) \cdot(3 / 2)^{k-1} \cdot 2^{k}|a|=3^{k-2} \cdot 2|a| .
\end{aligned}
$$

In particular, for every $a, b \in r$ the intersection $2 a \cap 2 b$ can be nonempty only for $b \in N(a)$, so that the cells $2 b, b \in r$, cover their union with multiplicity at most nine.

\subsubsection{The majorant $F$ possesses properties 1)-5) in the statement of the} main lemma. We mean the function (2.20):

$$
F=F_{r}=\sum_{b \in r} \varphi_{b}^{*}
$$

The multiplicity estimate for the covering $r$ implies that $F \in C^{\infty}(\mathbb{R})$ and $\left\|F^{\prime}\right\|_{\infty} \leq$ $9\left\|\varphi^{\prime}\right\|_{\infty}=A$; the support of $F$ is included in $(3 / 2) I^{*}$ (because $\varphi_{b}^{*} \equiv 0$ off $(3 / 2) b$ for every $b \in r$ ). The inequality $f \leq F$ (on $I^{*}$ ) is a consequence of the fact that the system $r$ covers $[-1 / 2,1 / 2)$ and all its elements are long. Next, from property (i) of the system $r$, estimate (2.19), and the inequality $\left|\varphi_{b}^{*}\right| \leq|b|$ we deduce that

$$
\begin{aligned}
\int_{\mathbb{R}} F & \leq \sum_{b \in r_{1}} \int_{2 b} \varphi_{b}^{*}+\sum_{b \in r_{2}} \int_{2 b} \varphi_{b}^{*} \leq \sum_{b \in A_{f}^{\max }}+\sum_{c \in A_{f}^{\max }} \sum_{b \in t(c)} \\
& \leq(16 k)^{-1} \sum_{b \in A_{f}^{\max }} \int_{b} f+\sum_{c \in A_{f}^{\max }} \sum_{b \in t(c)} 2|b|^{2} \\
& \leq(16 k)^{-1} \int_{I^{*}} f+12 \sum_{c \in A_{f}^{\max }}|S(c)| \leq \frac{1}{k} \int_{I^{*}} f
\end{aligned}
$$

(once again, we used the inequality $|S(c)| \leq(16 k)^{-1} \int_{c} f, c \in A_{f}$, at the last step). So, we have verified properties 1), 2), 3), and 5) for $F=F_{r}$.

2.6.8. Estimating the norm $\left\|\widetilde{F}^{\prime}\right\|_{\infty}$. If $x \in \mathbb{R}$ lies at a positive distance from $\bigcup_{b \in r} 2 b$ (say, to the right of $x^{*}=\sup \bigcup_{b \in r} 2 b$ ), then

$$
\left|\widetilde{F}^{\prime}(x)\right|=\frac{1}{\pi} \int_{-1}^{x^{*}} \frac{F(t) d t}{(x-t)^{2}} \leq \frac{1}{\pi} \int_{-1}^{x^{*}} \frac{F(t)}{\left(x^{*}-t\right)^{2}} d t=\left|\widetilde{F}^{\prime}\left(x^{*}\right)\right| \leq \sup _{\sup F}\left|\widetilde{F}^{\prime}\right|
$$

(the last identity follows from the fact that $F \equiv 0$ near $x^{*}$ ). Thus, it suffices to estimate $\left|\widetilde{F}^{\prime}(x)\right|$ only for $x \in \bigcup_{b \in r} 2 b$. Let $a=a(x)$ be a cell of the system $r$ with $x \in 2 a(x)$. Then

$$
\left|\widetilde{F}^{\prime}(x)\right| \leq I+I I+I I I,
$$




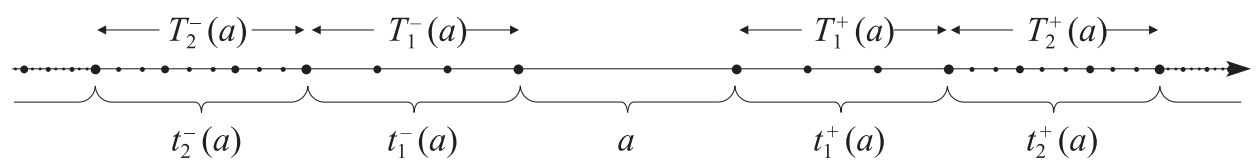

FiguRE 7.

where

$$
\begin{aligned}
I & =\sum_{b \in N(a)}\left|\widetilde{\varphi}_{b}^{* \prime}(x)\right| \leq 9\left\|\widetilde{\varphi}^{* \prime}\right\|_{\infty} \leq A, \\
I I & =\sum_{\substack{b \in r \backslash N(a),|b| \leq 2|a|}} \int_{2 b} \frac{\varphi_{b}^{*}(t)}{(x-t)^{2}} d t \leq 2|a| \cdot 9 \int_{|u| \geq|a| / 2} \frac{d u}{u^{2}}=72
\end{aligned}
$$

(because, by Subsection 2.6.6, $d(b, a) \geq|a| / 2$ in this sum, and the multiplicity of the system $\{2 b\}, b \in r$, is at most nine), and

$$
\begin{aligned}
I I I & =\sum_{k=2}^{\infty} \sum_{\substack{b \in r,|b|=2^{k}|a|}} \int_{2 b} \frac{\varphi_{b}^{*}(t)}{(x-t)^{2}} d t \leq \sum_{k=2}^{\infty} \sum_{\substack{b \in r,|b|=2^{k}|a|}} 2^{k}|a| \int_{2 b} \frac{d t}{(x-t)^{2}} \\
& \leq \sum_{k=2}^{\infty} 2^{k}|a| \cdot 9 \int_{|u| \geq 2 \cdot 3^{k-2}|a|} \frac{d u}{u^{2}}=108
\end{aligned}
$$

(again by Subsection 2.6.6).

We have proved the estimate $\left\|\widetilde{F}_{r}^{\prime}\right\|_{\infty} \leq A$, so that $F$ possesses all the properties promised in the main lemma. The matter depends now on a tiny thing: to explain the structure of the tails $t(a)$ and to construct the regular covering $r$.

2.7. Tails and regularization. We agree to denote by $\bar{l}$ the right end of a segment $l \subset \mathbb{R}$.

With every cell $a$ (not necessarily included in $I^{*}$ ), we associate its left and right tails $t^{-}(a)$ and $t^{+}(a)$, and then we put

$$
t(a)=t^{-}(a) \cup t^{+}(a)
$$

The right tail $t^{+}(a)$ is the union of finite series $t_{p}^{+}(a), p=1,2, \ldots$ of cells. The first series $t_{1}^{+}(a)$ consists of three cells of length $|a| / 2$ each; their union is the interval $T_{1}^{+}(a)$ of length $3|a| / 2$ adjacent to $a$ from the right: $T_{1}^{+}(a)=[\bar{a}, \bar{a}+3|a| / 2)$; the second series $t_{2}^{+}(a)$ contains 9 cells of length $|a| / 4$ each, and their union $T_{2}^{+}(a)$ is the interval $\left[\overline{T_{1}(a)}\right.$, $\left.\overline{T_{1}(a)}+9|a| / 4\right)$, and so on; finally,

$$
t^{+}(a)=\bigcup_{p=1}^{\infty} t_{p}^{+}(a)
$$

The "left" series $t_{p}^{-}(a)$ and the tail $t^{-}(a)$ are defined similarly (by symmetry relative to $a$ ). We observe that

$$
\sum_{b \in t(a)}|b|=2 \sum_{p=1}^{\infty}(3 / 2)^{p}|a|=+\infty
$$

so that the tail of $a$ covers $\mathbb{R} \backslash a$, and its part in $I^{*}$ covers $[-1 / 2,1 / 2)$. At the same time,

$$
\sum_{b \in t(a)}|S(b)|=2 \sum_{p=1}^{\infty}(3 / 4)^{p}|a|^{2}=6 S(a),
$$

and we obtain (2.21). 
To define the regular system $r$, we put

$$
\mathcal{B}_{f}=A_{f}^{\max } \cup \bigcup_{a \in A_{f}^{\max }} t(a)
$$

and then

$$
r=\left\{c \in \mathcal{B}_{f}^{\max }: c \subset I^{*}\right\} .
$$

We shall comment on this definition in Subsection 2.7.2; now we finish the proof of the main lemma.

2.7.1. The end of the proof of the main lemma. The system $r$ is a partition of the cell $I^{*}=[-1 / 2,1 / 2)$, because $\bigcup_{c \in \mathcal{B}_{f}^{\max }} c=\bigcup_{c \in \mathcal{B}_{f}} c=\mathbb{R}$. Put $\tau=A_{f}^{\max }, r_{1}=r \cap \tau$, $r_{2}=r \backslash r_{1}$. We verify statement (i) in Subsection 2.6.5. We must show that $r$ consists of long cells. If $c \in r_{1}$, then $c$ belongs to $\tau$ and, therefore, is long. But if $c \in r_{2}$, then $c \in t(a)$ for some $a \in \tau$, and therefore $|c|<|a| \leq 1$. In that case there is a cell $c^{\prime} \subset I^{*}$ such that $\left|c^{\prime}\right|=2|c|, c^{\prime} \supset c$, and if $c$ is not long, then $c^{\prime} \in A_{f}$, contradicting the fact that $c \in \mathcal{B}_{f}^{\max }$. This proves property (i) for the system $r$. We pass to (ii): suppose $a, b \in r$, $|a|=2^{k}|b|, k \geq 2$. We show that

$$
d(a, b) \geq(3 / 2)^{k-1}|a| .
$$

Indeed, $a \in r$ and $r \in \mathcal{B}_{f}$; therefore, $a$ "derives" from some cell $c \in A_{f}$ : either $a=c$, or $a \in t(c)$, whence $a \in t_{p}^{ \pm}(c), p=0,1,2, \ldots$ (we agree that $t_{0}^{ \pm}(c)=\{c\}$ ). Consider two cases: (i) $b$ derives from the same cell $c$, i.e., $b \in t(c)$; (ii) $b \notin t(c)$. In case (i), $b \in t_{p+k}^{ \pm}(c)$ for some $p \in \mathbb{Z}_{+}$, and the interval $T_{p+k-1}^{ \pm}(c)$ of length $(3 / 2)^{p+k-1}|c|$ lies between $a$ and $b$; but $|a|=|c| / 2^{p}$, and we arrive at (2.24):

$$
d(a, b) \geq(3 / 2)^{p+k-1}|c| \geq(3 / 2)^{k-1}|a| .
$$

We pass to case (ii). The tail $t(c)$ covers $\mathbb{R} \backslash c$, and consequently, covers $b$, because $b \cap c=\varnothing$. If a cell $l$ in $t(c)$ intersects $b$, then the strict inclusion $l \supsetneqq b$ is impossible because $l \in \mathcal{B}_{f}$ and $b \in \mathcal{B}_{f}^{\max }$. Thus,

$$
l \in t(c), l \cap b \neq \varnothing \Longrightarrow l \subset b,
$$

and $l$ is a union of elements of the tail $t(c)$. Let $l^{*}$ be the element of $t(c)$ nearest to $a$ and included in $b$. Then $d(a, b)=d\left(a, l^{*}\right)$ and $\left|l^{*}\right| \leq|b|=2^{-k}|a|$, whence $|a|=2^{m}\left|l^{*}\right|$ with $m \geq k \geq 2$, and the two cells $a, l^{*}$ derive from one and the same cell $c \in A_{f}$. By (i), we have $d\left(a, l^{*}\right) \geq(3 / 2)^{m-1}|a| \geq(3 / 2)^{k-1}|a|$. This finishes the proof.

2.7.2. Commentary. We try to make the construction of the system $r$ more transparent. For this, imagine some covering $\lambda$ of $\mathbb{R}$ by mutually disjoint dyadic cells (in brief, a dyadic partition) together with the family $\{S(a)\}_{a \in \lambda}$ of squares (we recall that $S(a)=a \times[0,|a|))$. The upper sides of these squares form the graph of some function $s^{\lambda}: \mathbb{R} \rightarrow(0,1]$. Its values are of the form $2^{-m}, m \in \mathbb{Z}_{+}$, and any set $\left\{s^{\lambda}=c\right\}$ is a union of cells of length $c$. Conversely, an arbitrary function with these properties is an $s^{\lambda}$ for some dyadic partition $\lambda$ of $\mathbb{R}$.

Let $\Lambda$ be a set of dyadic partitions. The upper envelope of the family $\left\{s^{\lambda}\right\}_{\lambda \in \Lambda}$, i.e., $\sup _{\lambda \in \Lambda} s^{\lambda}$, is $s^{\lambda^{0}}$, where $\lambda^{0}=\left(\bigcup_{\lambda \in \Lambda} \lambda\right)^{\text {max }}$. Perhaps, the graph of $s^{\lambda^{0}}$ is more illustrative than the partition $\lambda^{0}$ itself: $s^{\lambda^{0}}(x)$ is the height of the largest square $S(a)$ with $x \in a \in \lambda$.

We fix a cell $a$ and imagine the graph of $s^{t(a)}=s^{a}$. It is natural to think of this function as of a smoothed-out "lonely step" $|a| \chi_{a}$ ( $\chi_{E}$ stands for the characteristic function of a set $E$ ). At the exit of the cell $a$ outwards, this step falls abruptly, dropping suddenly from the height $|a|$ to zero. Yet the function $s^{a}$, coinciding with $|a| \chi_{a}$ on $a$, decays in a 
much lengthier manner, dragging the job out until infinity. Denoting by $a^{*}$ the standard cell $[-1 / 2,1 / 2)$, we have $s^{a}=\left(s^{a^{*}}\right)_{a}$ (i.e., this is the homothetic copy of the standard function $s^{a^{*}}$ adjusted to $a$; see Subsection 2.5.1). The function $s^{a^{*}}$ is even and decreases monotonically on the ray $[0,+\infty)$; for $|x| \geq 1 / 2, s^{a^{*}}(x)$ is the height of the square the base of which belongs to $t\left(a^{*}\right)$ and contains $x$; next, $s^{a^{*}}(x) \equiv 1$ on $a^{*}$. For large $|x|$, the quantity $s^{a^{*}}(x)$ is asymptotically equal to $A_{1}|x|^{-A_{2}}$ with $A_{2}=\log 3 / \log 2$.

Finally, to visualize the regularization $r$ of the system $\tau=A_{f}^{\max }$ (see (2.23)), imagine the chain of the squares $S(a), a \in \tau$. It can be highly irregular, with numerous and chaotic pits. The upper envelope of the family $\left\{s^{a}\right\}_{a \in \tau}$ is $s^{r(\tau)}$, where $r(\tau)$ is a certain dyadic partition of $\mathbb{R}$. The part of $r(\tau)$ that covers $a^{*}=[-1 / 2,1 / 2)$ is precisely the system $r$ defined in (2.23). The function $s^{r(\tau)}$ arises after application of a certain levelling procedure to the function $g$ generated by the dyadic partition $\tau=A_{f}^{\max }$ of $a^{*}(g(x)=|a|$ if $x \in a, a \in \tau)$. The graph of $g$ may have deep and irregular pits reflecting the troublesome behavior of the Lipschitz function $f$ : we know that tiny cells $b \in \tau$ may concentrate near a big cell $a \in \tau$. But under the passage from $\tau$ to $r$ they will be absorbed by cells in the tail $t(a)$.

Some cells belonging to $A^{\max }$ still belong to $r$ (for example, so do all cells of maximal length), but also some new cells are added. We have seen that all cells in $r$ remain long, but some become inessential, which might undermine the upper estimate of $\int_{\mathbb{R}} F_{r}$ in terms of $\int_{I^{*}} f$. However, this estimate survives thanks to (2.21); see Subsection 2.6.7. The levelling procedure has a wholesome effect on the Hilbert transform, reducing $\left\|\widetilde{F}_{r}^{\prime}\right\|_{\infty}$ compared to $\left\|\sum_{a \in \tau} \widetilde{\varphi}_{a}^{* \prime}\right\|_{\infty}$, but this feature is not so apparent (see Subsection 2.6.8).

\section{§3. Supplements, COMments, And technical Details}

In Subsection 3.1, we explain at last the meaning of the term "multiplier", which occurs in the title of the paper. For this we resort to a complex analysis interpretation of Theorem BM1. Next, in Subsection 3.2, we discuss some links between this theorem and other problems in analysis. Among these links, apparently, the main one is the relationship with Theorem BM2, which shall be discussed in Subsection 3.3. This discussion is followed by a concise summary of proofs of Theorem BM1 that appeared prior to our paper. A large part of the present section is occupied by Subsection 3.5 containing a review of the results presented in [HMI] and [HMII], and in some subsequent papers. All these results are about majorants admissible with respect to so-called "model" (or shiftcoinvariant) subspaces in the Hardy class $H^{2}$; the Paley-Wiener spaces are among them. The approach to Theorem BM1 presented in $\S 1$ arose in [HMI and [HMII precisely in this ("model") context. In the final Subsection 3.6, we collect diverse proofs (basically, for auxiliary facts) skipped in $\S \S 1$ and 2 ; also, we give some comments to $\S 2$.

3.1. Theorem BM1 as a fact of complex analysis. Theorem BM1 can be perceived as a statement about entire functions in the Cartwright class.

Let $\sigma$ be a positive number. We denote by Cart $_{\sigma}$ the set of all entire functions $f$ of finite degree $\sigma$ (i.e., $\log |f(\zeta)|=O\left(|\zeta|^{\sigma+\varepsilon}\right),|\zeta| \rightarrow \infty$, for every $\varepsilon>0$ ) such that

$$
\log ^{+}|f| \mid \mathbb{R} \in L^{1}(P),
$$

where, as usual, $d P(x)=\pi^{-1}\left(1+x^{2}\right)^{-1} d x$. The set Cart $:=\bigcup_{\sigma>0}$ Cart $_{\sigma}$ is called the Cartwright class. This class plays an important role in complex and harmonic analysis; see [Ls, Koo1, Koo2, Koo3, Lv, [HJ]. In particular, Cart includes the class $B_{\sigma}$ of all entire functions of degree at most $\sigma$ and bounded on $\mathbb{R}$ (consequently, the Fourier image of any complex measure with compact support in $\mathbb{R}$ belongs to Cart). If $f$ is a nonzero function in the Cartwright class, then not only $\log ^{+}|f|$, but also $|\log | f||$ is integrable 
against the Poisson measure $P$, so that an arbitrary entire function of the form $f_{1} / f_{2}$ with $f_{1}, f_{2} \in$ Cart is itself in Cart. In [BM1, Beurling and Malliavin showed that Cart coincides with the set of such ratios, where $f_{1}, f_{2} \in B_{\sigma}$ for some $\sigma>0$. More precisely, the following is true.

Multiplier theorem. For every $f \in$ Cart and every $\varepsilon>0$, there exists $m \in B_{\varepsilon}$ such that the function $f m$ is bounded on $\mathbb{R}$.

The term "multiplier" refers to the function $m$.

3.1.1. Relationship between the multiplier theorem and Theorem BM1. In the multiplier theorem, there is no loss of generality in assuming that $|f(x)| \geq 1$ for every $x \in \mathbb{R}$. Indeed, putting $f^{*}(z)=\overline{f(\bar{z})}$, we see that $F:=f f^{*}+1 \in$ Cart. Clearly, $F(x) \geq 1$ for $x \in \mathbb{R}$, and if we find a multiplier $m \in B_{\varepsilon}$ such that $F m$ is bounded on $\mathbb{R}$, then $f m$ will also be bounded because $|f(x)| \leq|F(x)|$ if $x \in \mathbb{R}$ and $|f(x)| \geq 1$.

So, the multiplier theorem can be restated as follows.

If $f \in$ Cart and $|f(x)| \geq 1$ for every $x \in \mathbb{R}$, then $1 /|f|$ is a fully admissible majorant (see the definition in Subsection 1.3).

Indeed, the former statement implies the latter, because $f m(\sin \varepsilon z / z) \in P W_{\sigma+2 \varepsilon}$ if $f \in \mathrm{Cart}_{\sigma}, m \in B_{\varepsilon}$; at the same time, the latter statement implies the former because $P W_{\sigma}$ and $H_{\sigma}^{2}$ are included in $B_{\sigma}$.

Beurling and Malliavin proved the multiplier theorem and the statement called here "Theorem BM1" separately, deducing the two results from a general "energy" statement (see Subsection 3.4 below). However, as has been shown by Koosis, the multiplier theorem is equivalent to Theorem BM1.

In order to briefly describe Koosis' result, consider two classes $M_{1}$ and $M_{2}$ of majorants (i.e., nonnegative functions defined on $\mathbb{R}$ and not exceeding 1). The class $M_{1}$ is said to prop up $M_{2}$ if for every $\omega_{2} \in M_{2}$ there exists $\omega_{1} \in M_{1}$ with $\omega_{1} \leq \omega_{2}$. Clearly, if $M_{1}$ props up $M_{2}$ and consists of fully admissible majorants, then all functions in $M_{2}$ are also fully admissible. Koosis [Koo3, Chapter IV] considered the classes

$$
\begin{aligned}
& M_{1}=\left\{\omega: 0<\omega \leq 1, \log \omega \in L^{1}(P) \cap \operatorname{Lip}_{1}(\mathbb{R})\right\}, \\
& M_{2}=\{\omega=1 /|f|: f \in \text { Cart },|f| \geq 1 \text { on } \mathbb{R}\}
\end{aligned}
$$

and showed that they prop up each other, which implies the equivalence of Theorem BM1 and the multiplier theorem. It should be noted that the classes $M_{1}$ and $M_{2}$ are quite different despite the fact that they prop up each other: it is not true that $M_{j} \subset M_{k}$ for $j \neq$ $k$. This is clear if $j=1$, and for $j=2$ the inclusion is disproved radically by the following example: $\omega=1 /|f|, f=1+\prod_{k \geq 0}\left(1-z / 2^{k}\right)^{2}, z \in \mathbb{C}$. Indeed, $|f(z)| \leq 1+(g(|z|))^{2}$, where $g(u)=\prod_{k \geq 0}\left(1+u / 2^{k}\right), u \geq 0 ; g(u)=(1+u) g(u / 2)=(1+u)(1+u / 2) \cdots$ $\left(1+u / 2^{N}\right) g\left(u / 2^{N+1}\right) \leq(1+u)^{N} \cdot M$, where $N=[\log u / \log 2], M=\max _{[0,1]} g$. Therefore, $|f(z)|=O\left(|z|^{c \log |z|}\right), c>0,|z| \rightarrow \infty$, and $f$ is an entire function of zero order (a fortiori, it belongs to the Cartwright class). At the same time, for $x=2^{m}, m=1,2, \ldots$, we have $\log \omega(x)=0$ and $\log \omega(x+1) \geq 2 \sum_{k \geq 0} \log \left|1-(x+1) / 2^{k}\right|=\sum_{k \leq m}+\sum_{k \geq m+1} \geq$ $c m^{2}+O(1)$ as $m \rightarrow+\infty$. We see that $\varlimsup_{x \rightarrow+\infty}(\Omega(x+1)-\Omega(x))=+\infty$, and $\omega \notin M_{1}$ (the function $\Omega$ even fails to be uniformly continuous).

3.1.2. The relationship with functions of bounded characteristic and the M. G. Kreйn theorem. The Nevanlinna class in a domain $G \subset \mathbb{C}$ is the set of all functions analytic in $G$ and representable as the ratio of two bounded analytic functions: $N(G)=\left\{f / g: f, g \in H^{\infty}(G), g\right.$ has no zeros in $\left.G\right\}$. We need the classes $N_{ \pm}=N\left(\mathbb{C}_{ \pm}\right)$. M. G. Kreln showed that they are closely related to the Cartwright class. Every function 
$f \in N_{+}$has the boundary values

$$
f(x):=\lim _{y \downarrow 0} f(x+i y),
$$

for a.e. $x \in \mathbb{R}$, and

$$
\int_{\mathbb{R}}|\log | f(x)|| d P(x)<+\infty
$$

if $f \not \equiv 0$. Also, we have the following:

$$
f \in N_{+} \Longleftrightarrow \begin{aligned}
& f \text { is analytic in } \mathbb{C}_{+} \text {and } \log ^{+}|f| \text { has a har- } \\
& \text { monic majorant in } \mathbb{C}_{+} \text {(see [HJ, p. 192]). }
\end{aligned}
$$

Theorem (M. G. Krĕn $[\mathrm{Kr}]$ ). An entire function $f$ belongs to the Cartwright class if and only if $f \mid \mathbb{C}_{+} \in N_{+}$and $f \mid \mathbb{C}_{-} \in N_{-}$.

Thus, the relation $f \in$ Cart means that the two pieces $f_{ \pm}:=f \mid \mathbb{C}_{ \pm}$of $f$ admit representations

$$
f_{ \pm}=a_{ \pm} / b_{ \pm} \quad \text { in } \mathbb{C}_{ \pm}, \quad \text { where } a_{ \pm}, b_{ \pm} \in H^{\infty}\left(\mathbb{C}_{ \pm}\right) .
$$

Now we observe that if $f$ is an entire function and $f=A / B$, where $A, B \in B_{s}$, then $f$ admits a representation (3.1) of a special kind (the "pieces" $a_{ \pm}, b_{ \pm}$are cut out of the entire functions $A, B$ multiplied by exponentials):

$$
f \mid \mathbb{C}_{ \pm}=e^{ \pm i s z} A / e^{ \pm i s z} B
$$

(note that $e^{ \pm i s z} A\left|\mathbb{C}_{ \pm}, e^{ \pm i s z} B\right| \mathbb{C}_{ \pm} \in H^{\infty}\left(\mathbb{C}_{ \pm}\right)$), so that, in a sense, the representation $\left(3.1^{\prime}\right)$ becomes global (derives from entire $A$ and $B$ ). The multiplier theorem says that an arbitrary function in the Cartwright class is representable in the special form $\left(3.1^{\prime}\right)$. Specifically, if $f \in \mathrm{Cart}_{\sigma}$, we can put $A=f m, B=m$, where $m \in B_{\varepsilon} ;\left(3.1^{\prime}\right)$ is true with $s=\sigma+\varepsilon$.

3.2. Some connections and applications of Theorem BM1. The relationship between Theorem BM1 and the uncertainty principle in Fourier analysis was discussed already in the Introduction (Subsection 0.5): different approaches to this theorem lead to different construction methods (or at least existence proofs) for a function $f$ with bounded spectrum and small modulus $|f|$ not exceeding a given majorant $\omega$.

Applications of Theorem BM1 to various problems of analysis were discussed in a large portion of the book Koo2 (Chapter X in it is entitled "Why we want to have multiplier theorems"). Some applications treated there pertain to estimates for the Poisson integral of trigonometric sums with a spectral gap. For instance, on pp. 210-211 the following problem was solved: for given $a>0$ and a weight $w \in L^{1}(\mathbb{R}, d x), w \geq 0$, consider the functional $S \mapsto P_{\beta}(S)$, where $S$ runs through all trigonometric polynomials with spectrum off $(-a, a)$, and

$$
P_{\beta}(S)=(\operatorname{Im} \beta / \pi) \int_{\mathbb{R}} S(t)|t-\beta|^{-2} d t, \quad \beta \in \mathbb{C}_{+} .
$$

It is required to describe all weights $w$ for which this functional is continuous in the norm of $L^{2}(w(x) d x)$ for some $\beta \in \mathbb{C}_{+}$. This problem turns out to be equivalent to the admissibility of some majorant related to $w$ (admissibility is understood in a certain integral sense). On p. 272 of [Koo2, a relationship of our theme with the existence of the weighted estimate

$$
\int_{\mathbb{R}}|\widetilde{S}|^{2} \omega^{2} d x \leq C(\omega, w) \int_{\mathbb{R}}|S|^{2} w d x
$$

was established, where $S$ is still an arbitrary trigonometric polynomial with spectrum in $\mathbb{R} \backslash(-a, a)$, the weight $w \in L^{1}(d x)$ is fixed, and $\omega$ is a nonzero weight to be found. Also 
in this case, the problem is solvable if and only if the weight $w \cdot\left(1+x^{2}\right)$ is admissible in a certain integral sense.

The Beurling-Malliavin multiplier theorem is also related to classical problems of weighted approximation by polynomials and entire functions of exponential type on $\mathbb{R}$ and on discrete subsets of it ("Bernstein's problem"). See the discussion on pp. 468-469 in Koo2, and also on p. 174 in Koo3. We specially mention criteria of normality for families $\{f\}$ of polynomials or entire functions of exponential type in terms of "logarithmic sums" $\sum_{k}\left(\log ^{+}\left|f\left(\lambda_{k}\right)\right|\right) /\left(1+\lambda_{k}^{2}\right)$, where $\left(\lambda_{k}\right)$ is a sequence of points in $\mathbb{R}$. This theme is also related to Theorem BM1; see Koo7, KooP, P.

For other connections and applications of Theorem BM1, see Chapter X in Koo2] mentioned above, and also Chapter 3 in the monograph DeB.

Now we turn to Theorem BM2 which, apparently, was the main motivation behind the work described in BM1.

Our purpose in Subsection 3.3 is only a cursory revision of the relationship between Theorems BM1 and BM2; we leave apart the vast range of problems adjacent to the content of [BM2].

3.3. On the second theorem of Beurling and Malliavin. Let $R$ be a positive number, and $\Lambda$ a discrete set of reals. For which pairs $(R, \Lambda)$ is the family $E(\Lambda)=$ $\left(e^{2 \pi i \lambda x}\right)_{\lambda \in \Lambda}$ dense in $L^{2}(-R, R)$ ? Experience shows that, in this wording, the question is "too strict" and does not admit an anywhere near transparent answer that is complete at the same time. The problem simplifies if, in place of the strict prescription of the number $R$, we turn to the search (for $\Lambda$ fixed) of the lowest upper bound $R(\Lambda)$ for the set $\left\{r>0: E(\Lambda)\right.$ is dense in $\left.L^{2}(-r, r)\right\}$. The second Beurling-Malliavin theorem (Theorem BM2 for short) expresses $R(\Lambda)$ in terms of a purely geometric characteristic of $\Lambda-\mathrm{a}$ certain "density" of it at infinity. In the subsequent discussion, we restrict ourselves only to sets $\Lambda$ of reals, though Theorem BM2 is also applicable to sets $\Lambda \subset \mathbb{C}$. We note also that the choice of the spaces $L^{2}(-r, r)$ is accidental, they may be replaced by the spaces $C([-r, r])$, etc. Those who wish to read more widely about Theorem BM2 may refer to BM2, Koo2, Koo3, HJ]; a new approach to that theorem was developed in MP. Here we only discuss the relationship between Theorems BM1 and BM2, without reproducing the Beurling-Malliavin formula for $R(\Lambda)$, which has the form $R(\Lambda)=\operatorname{BM}(\Lambda)$, where $\operatorname{BM}(\Lambda)$ is the "Beurling-Malliavin density" of $\Lambda$ at infinity, the definition of which will not be written out here. The proof of the last identity splits into two parts. First, under the assumption $r<\operatorname{BM}(\Lambda)$, we must check that the system $E(\Lambda)$ is dense in $L^{2}(-r, r)$, i.e., show that every function $\varphi \in L^{2}(\mathbb{R})$ vanishing on $\mathbb{R} \backslash(r, r)$ and such that $\hat{\varphi} \mid \Lambda=0$, is zero. In other words, we must prove that

$$
\psi \in P W_{2 \pi r}, \quad \psi \mid \Lambda=0 \Longrightarrow \psi=0
$$

if $r<\operatorname{BM}(\Lambda)$. This part of the proof is relatively simple. The main difficulties pertain to the second part, where for arbitrary $r>\operatorname{BM}(\Lambda)$ we must construct a nonzero function $\psi \in P W_{2 \pi r}$ vanishing on $\Lambda$ (by the Paley-Wiener theorem, its Fourier preimage is supported on $[-r, r]$ and is orthogonal to the family $E$ ).

It is here that Theorem BM1 comes to our aid, allowing us, instead of $P W_{2 \pi r}$, to work with the much wider Cartwright classes $\operatorname{Cart}_{2 \pi r}$ in the last problem. Indeed, take $r^{\prime} \in(\operatorname{BM}(\Lambda), r)$ and suppose we are able to construct a nonzero function $\varkappa \in \operatorname{Cart}_{2 \pi r}$ vanishing on $\Lambda$. By the multiplier theorem (see Subsection 3.1.1), there exists a nonzero function ("multiplier") $m \in B_{\varepsilon}$, where $\varepsilon=\pi\left(r-r^{\prime}\right)$, such that $\varkappa m \in B_{2 \pi r}$. So, the function $\varkappa m \sin \varepsilon z / z$ vanishes on $\Lambda$, belongs to $P W_{2 \pi r}$, and is not identically zero.

In essence, in the second part of the proof of Theorem BM2, we again encounter an admissibility (more precisely, $2 \pi r$-admissibility) problem, but this time for majorants $\omega$ of 
the special form $\omega=\chi_{\mathbb{R} \backslash \Lambda}$, where $\Lambda$ is a discrete subset of $\mathbb{R}$. The inequality $r>\operatorname{BM}(\Lambda)$ turns out to be a sufficient condition (also sharp in a sense) for the admissibility of such a majorant $\omega$. But $\omega$ is not covered by Theorem BM1: it is discontinuous and (in the first place!) vanishes somewhere. Theorem BM1 interferes in the admissibility proof for such $\omega$ 's only in an indirect way. It would be interesting to find an approach to the admissibility problem suitable for the two classes of majorants, either positive as in Theorem BM1, or vanishing on a discrete set as in Theorem BM2. The reader may consult Koo2, Koo3, HJ] for references concerning Theorem BM2, including the work of Khabibullin, Krasichkov-Ternovskiu, J.-P. Kahane, Redheffer; we mention the recent paper $[\mathrm{MP}]$ once again.

3.4. Comments on the proofs of Theorem BM1. Presently, along with the pioneering paper BM1, the main sources pertaining to the question under discussion are the books [Koo1, Koo2], and [Koo3], where references to other publications due to Koosis can be found. The proof presented in [HJ develops mainly as in BM1, but involves some simplifications taken from $\mathrm{Mal}$. The proof in Koo3 employs the so-called "fundamental lemma" of Beurling and Malliavin about the ratio $u(x, y) / y$ for positive functions $u$ harmonic in $\mathbb{C}_{+}$; see [Koo3, p. 64]. In this monograph the reader can find references to the proofs of that lemma invented by Kargaev, Sjögren, and Nazarov. Each of these proofs leads, in the long run, to a new proof of Theorem BM1. We recall once again the approach to that theorem related to estimates of logarithmic sums for polynomials and entire functions of exponential type; see Koo7, KooP.

The main result of the paper [BM1] covered both Theorem BM1 (as stated in Subsection 0.2) and the multiplier theorem (see Subsection 3.1). Precisely these two statements became widely known. Moreover, the reading of BM1 suggests that the authors of that paper treated their most general results merely as a tool for the proof of Theorem BM1 and the multiplier theorem, which were viewed as principal: the general result was even not mentioned in the introductory description of the paper and appeared at the end as a remark. In our opinion, that result is interesting for its own sake, and we regret to note that, as yet, we have not managed to prove it by using our elementary approach, which leads directly to Theorem BM1 and to the equivalent multiplier theorem.

We dwell on more details concerning the fundamental paper BM1. The problem discussed there was posed not quite in the same way as in the subsequent papers on the same topic (including the present one). In order to reproduce the original setting, consider a function $\omega \in L^{\infty}(\mathbb{R}), 0 \leq \omega \leq 1$, and put

$$
L_{\omega}^{p}:=\left\{f: f \text { is measurable on } \mathbb{R} \text {, and } f / \omega \in L^{p}(d x)\right\},
$$

where $p \in[1,+\infty]$. The main problem in BM1 looks like this: to describe the class $W_{p}$ of weights $\omega$ such that

(a) the space $L_{\omega}^{p}$ is translation invariant, and

(b) $L_{\omega}^{p}$ contains a nonzero function with spectrum of finite (of given) length.

In BM1, this compound question was answered exhaustively: $W_{p}$ does not depend on $p$ and coincides with the set $W$ of all measurable weights $\omega, 0<\omega \leq 1$, for which $\mathcal{L}(\omega)>-\infty$, and

$$
\operatorname{ess} \sup |\Omega(x+t)-\Omega(x)|<+\infty \quad \text { for every } t \in \mathbb{R} ;
$$

if $\omega \in W$, then the width of the spectrum of a nonzero function $f \in L_{\omega}^{p}$ can be made arbitrarily small. As usual, here $\Omega=|\log \omega|$.

Surely, the main interest lies in condition (b) in its pure form rather than in condition (a) (trivially equivalent to condition $(*)$ ). Separating (b) from (a), for $p=+\infty$ we arrive at the problem of full admissibility for $\omega$, as stated in Subsection 1.3. If $p$ is finite, we 
obtain an integral version of the $\sigma$-admissibility problem: given a measurable function $\omega, 0<\omega \leq 1$, construct a nonzero function $f \in L_{\omega}^{p}$ with spectrum of width at most $\sigma$. We remind the reader that, in admissibility problems, there is no loss of generality in replacing condition $(*)$ with the condition $\Omega \in \operatorname{Lip}_{1}(\mathbb{R})$.

If we lift the disguising condition (a), condition (b) immediately becomes hardly translatable into the usual language expressing the "level behavior" of $\omega$, which, in combination with the finiteness of the logarithmic integral $\mathcal{L}(\Omega)$, would be equivalent to full admissibility.

A deep and detailed discussion of this "level behavior" problem (even beyond the framework of the Lipschitz condition) can be found at the end of the monograph [Koo2]. It creates an impression that it is hardly possible to convey the desired feature unbiasedly in reasonable terms.

Now we quote the general result of the paper BM1, which includes both Theorem BM1 and the multiplier theorem. The letters $\omega$ and $\Omega$ will have the usual meaning. We put $k=\Omega / x$ (without loss of generality, we assume that $\Omega \equiv 0$ near the origin). In the statements below, the following integral will occur:

$$
\mathcal{E}(k):=\iint_{\mathbb{R} \times \mathbb{R}}\left(\frac{k(x)-k(y)}{x-y}\right)^{2} d x d y .
$$

The finiteness of it means that the harmonic extension $u$ of $k$ to the half-plane $\mathbb{C}_{+}$(with the help of the Poisson integral) has finite Dirichlet integral $\int_{\mathbb{C}_{+}}|\nabla u|^{2} d x d y$.

The first statement below describes some majorants $\omega$ admissible in an integral sense.

Theorem (see [BM1]). If $\mathcal{L}(\Omega)<+\infty$ and $\mathcal{E}(k)<+\infty$, then for every $p \in[1,+\infty)$ there exists a nonzero function $f$ in $L_{\omega}^{p}$ with spectrum of arbitrarily small width.

For $p=\infty$ (i.e., in the problem of "pure", not integral admissibility), the result of BM1 can be stated as follows.

Theorem. If the integrals $\mathcal{L}(\Omega)$ and $\mathcal{E}(k)$ are finite, then for every $\varepsilon>0$ the following majorant $\omega_{\varepsilon}$ is fully admissible:

$$
\omega_{\varepsilon}(x):=|\operatorname{Ext} \omega|(x+i \varepsilon)=\exp \varepsilon \int_{\mathbb{R}}\left(\log \omega /\left(\pi|t-(x+i \varepsilon)|^{2}\right)\right) d t .
$$

This theorem guarantees the full admissibility of some regularization $\omega_{\varepsilon}$ rather than of $\omega$ itself. If $\Omega \in \operatorname{Lip}_{1}(\mathbb{R})$, the subscript $\varepsilon$ can be dropped.

It is impossible to present here an anywhere near detailed analysis of all proofs of Theorem BM1, and we restrict ourselves to general remarks.

Starting with BM1 (but not in the present paper), the required function $f$ with bounded spectrum and subordinate to $\omega$ (i.e., such that $|f| \leq \omega$ or $f \in L_{\omega}^{p}$ ) was always thought of as an entire function of finite degree, and even more specifically, as a certain canonical product. The inequality $|f| \leq \omega$ leaves enough room for assuming that $|f|$ is $\exp U^{\mu}$, where $U^{\mu}$ is the logarithmic potential of some nonnegative measure $\mu$ on $\mathbb{R}$, which is not necessarily discrete: having ensured the inequality $U^{\mu} \leq-\Omega$, we may convert the function $\exp \left(U^{\mu}+i \tilde{U}^{\mu}\right)$ into a genuine canonical product by "discretization" of $\mu$ - it suffices to replace $\mu$ with a pure point measure in quite a crude way.

The subsequent procedures can be described (quite broadly) as follows: now our purpose is the search of the potential $U^{\mu}$, and at the beginning this potential should be perceived simply as a function harmonic in $\mathbb{C} \backslash \mathbb{R}$ and satisfying certain boundary conditions on $\mathbb{R}$ (we do not reproduce them but remind the reader that $f=\exp \left(U^{\mu}+i \tilde{U}^{\mu}\right.$ ) must be a function of exponential type). There are two well-trodden roads towards the solution of such problems: Dirichlet's (energy) method and Perron's method (the analysis of sub- and superharmonic majorants). Nearly all previous proofs of Theorem BM1 fall 
within one of these patterns. Energy considerations were employed in the pioneering proof, and also in the proof in the book [DeB]; the paper [Mal] and the exposition in [HJ] should also be attributed to this group.

In reference to the final statements (i.e., to Theorem BM1 and the multiplier theorem), the energy terminology sounds like something external and alien. This was a motivation for Koosis to choose another way, basically of Perron type. In [Koo3, pp. 222-224] the reader may find quite an expressive piece of writing about the quest for elimination of the energy techniques, as well as a review of the proofs of Theorem BM1 known at that time together with the indication of the fact that Nazarov's proof of the "fundamental lemma" mentioned above had finally led to a proof of Theorem BM1 that was free of energy considerations. The same feature is characteristic of our proof, which involves neither potential theory, nor complex analysis. Already in [HMI, HMII], our approach was aimed not merely at Theorem BM1, but rather at its analogs for model subspaces of the Hardy space and for the de Branges spaces of entire functions. A short survey of this theme will be given in the next subsection.

3.5. A generalization: majorants admissible for model subspaces. The approach to Theorem BM1 presented in $\S \S 1$ and 2 first arose in [HMI, HMII, where the Paley-Wiener spaces $P W_{\sigma}$ appeared as quite a particular case of model subspaces in $H^{2}(\mathbb{R})$. In this subsection we give a concise review of some results of [HMI, HMII] and of some subsequent papers. In distinction with $\S 1$, we shall leave the real line and, along with the Hardy classes $H^{p}(\mathbb{R})$, consider the classes $H^{p}\left(\mathbb{C}_{+}\right)$. Recall that, for $p \in(0,+\infty)$,

$$
f \in H^{p}\left(\mathbb{C}_{+}\right) \Longleftrightarrow f \text { is analytic in } \mathbb{C}_{+} \text {and } \sup _{y>0} \int_{-\infty}^{+\infty}|f(x+i y)|^{p} d x<+\infty,
$$

and $H^{\infty}\left(\mathbb{C}_{+}\right)$is the set of all functions bounded and analytic in $\mathbb{C}_{+}$. The class $H^{p}(\mathbb{R})$ can be perceived as the set of traces of all functions of class $H^{p}\left(\mathbb{C}_{+}\right)$on the boundary:

$$
f \in H^{p}(\mathbb{R}) \Longleftrightarrow f(x)=\lim _{y \downarrow} f(x+i y) \text { for a.e. } x \in \mathbb{R}
$$

for some function $f$ in $H^{p}\left(\mathbb{C}_{+}\right)$; for $p \geq 1$, the norm in $H^{p}\left(\mathbb{C}_{+}\right)$is inherited from $H^{p}(\mathbb{R})$ (so, finally, from $L^{p}(\mathbb{R})$ ). In particular, $H^{2}\left(\mathbb{C}_{+}\right)$becomes a Hilbert space, which may be identified with $H^{2}(\mathbb{R})$. The theory of the Hardy classes $H^{p}\left(\mathbb{C}_{+}\right)$is presented in [HJ, Koo4, N, D, Ga, Hof].

A function $\theta \in H^{\infty}\left(\mathbb{C}_{+}\right)$is said to be inner if $|\theta(x)|=1$ for a.e. $x \in \mathbb{R}$. Inner functions are exemplified by the harmonics $e^{i \sigma z}, \sigma>0$, or by the Blaschke products $B$,

$$
B(z)=\prod_{k=1}^{\infty} \frac{1-z / z_{k}}{1-z / \bar{z}_{k}}
$$

where the sequence $\left(z_{k}\right)$ of points of $\mathbb{C}_{+}$satisfies the Blaschke condition

$$
\sum_{k=1}^{\infty} \frac{\operatorname{Im} z_{k}}{\left|z_{k}\right|^{2}}<+\infty,
$$

and $\lim _{k \rightarrow \infty}\left|z_{k}\right|=\infty$ (the function $B$ should be called a meromorphic Blaschke product; indeed, it is defined in the complement of the sequence $\left\{\bar{z}_{k}\right\}_{k=1}^{\infty}$ and is meromorphic; we shall not need more general Blaschke products here).

Every inner function $\theta$ gives rise to the so-called model subspace $K_{\theta}$ of $H^{2}\left(\mathbb{C}_{+}\right)$:

$$
K_{\theta}:=H^{2}\left(\mathbb{C}_{+}\right) \ominus \theta H^{2}\left(\mathbb{C}_{+}\right) .
$$

By the well-known Beurling theorem, if $\theta$ runs through all inner functions, then the sets $\theta H^{2}\left(\mathbb{C}_{+}\right)$run through the class of all subspaces of $H^{2}\left(\mathbb{C}_{+}\right)$invariant under multiplication 
by the harmonics $e^{i \sigma z}, \sigma>0$. For this reason, the spaces $K_{\theta}$, i.e., the orthogonal complements of the spaces $\theta H^{2}\left(\mathbb{C}_{+}\right)$, are often called coinvariant under multiplication by $e^{i \sigma z}$, $\sigma>0$. We prefer the shorter term "a model subspace", introduced by N. K. Nikol'skii and indicating the role played by vector and operator analogs of the spaces $K_{\theta}$ in the construction of the Nagy-Foiaş functional model for Hilbert space contractions; see, e.g., [N].

The spaces $K_{\theta}$ (and their natural analogs in the unit disk) arise in complex and harmonic analysis and operator theory for various and numerous reasons. The spaces $K_{\theta}$ corresponding to a meromorphic inner function (i.e., $\theta=e^{i \sigma z} B$, where $\sigma>0$ and $B$ is a meromorphic Blaschke product) are closely related to the de Branges Hilbert spaces of entire functions (see $[\mathrm{DeB}]$ ), and this will be discussed below. Finally, we note that the Paley-Wiener space $P W_{\sigma}$ (see Subsection 1.2) coincides with $e^{-\pi i \sigma z} H_{2 \sigma}^{2}=$ $e^{-\pi i \sigma z} K_{e^{4 \pi i \sigma z}}$.

A nonnegative function continuous on $\mathbb{R}$ will be called a majorant. A majorant $\omega$ is said to be admissible for $K_{\theta}$ (for short, $\theta$-admissible; in symbols, $\omega \in \operatorname{Adm} \theta$ ) if there is a nonzero function $f \in K_{\theta}$ such that

$$
|f(x)| \leq \omega(x)
$$

for all $x \in \mathbb{R}$. In this case, $f$ is said to be subordinate to $\omega$. If $f \in K_{\theta}$ and $f \not \equiv 0$, then $|f| \in \operatorname{Adm} \theta$.

For $\omega \in \operatorname{Adm} \theta$, the logarithmic integral

$$
\mathcal{L}(\Omega)=\int_{\mathbb{R}} \Omega^{+} d P
$$

where $\Omega=-\log \omega, \Omega^{+}=\max (\Omega, 0)$, must be finite. However, the converse is not true in general, much for the same reason as in $\S 1$ : the functions in $K_{\theta}$ bear "higher analyticity" than those in $H^{2}(\mathbb{R})$ - the moduli of the latter are completely characterized by square integrability and the convergence of the logarithmic integral. Specifically, an arbitrary function $f \in K_{\theta}$ admits "analytic pseudocontinuation" to the lower half-plane $\mathbb{C}_{-}$in the sense that $f$ coincides a.e. on $\mathbb{R}$ with the function $\theta(x) \overline{g(x)}$ for some $g \in H^{2}\left(\mathbb{C}_{+}\right)$, so that $\theta \bar{g}$ is the trace on the boundary (on $\mathbb{R}$ ) of a function meromorphic in $\mathbb{C}_{-}$. This refers to an arbitrary inner function $\theta$; but if $\theta$ is meromorphic, then the functions in $K_{\theta}$ can be continued analytically across $\mathbb{R}$ (in the literal sense) up to functions meromorphic in $\mathbb{C}$ (the poles of such meromorphic functions lie in the set of poles of the Blaschke product incorporated in $\theta$ ). The above discussion hints that a $\theta$-admissible majorant must be fairly "regular"; excessive oscillations ("pits") on $\mathbb{R}$ are forbidden (though, by themselves, they may be compatible with the inequality $\mathcal{L}(\Omega)<\infty$ ). The situation is further aggravated compared to the classical case of Theorem BM1: even if $\mathcal{L}(\Omega)<\infty$ and the majorant is highly regular (say, is even and monotone decreasing on $[0,+\infty)$ ), it may fail to be $\theta$-admissible.

The situation is precisely such in the (extreme) case where $\theta$ is a finite Blaschke product with zeros $z_{1}, \ldots, z_{n} \in \mathbb{C}_{+}$. Then $K_{\theta}$ is the set of rational fractions $P / Q$, where

$$
Q=\left(z-z_{1}\right)\left(z-z_{2}\right) \cdots\left(z-z_{n}\right)
$$

and $P$ is an arbitrary polynomial of degree at most $n-1$ : the majorant $\frac{1}{(1+|x|)^{(n+1)}}$ is not $\theta$-admissible. It should be noted that the $\theta$-admissible majorant $(1+|x|)^{-n}$ is minimal in $\operatorname{Adm} \theta$ : any substantially smaller majorant $\omega$ (e.g., a majorant obeying $\omega(x)=o\left(|x|^{-n}\right.$ ) as $|x| \rightarrow \infty)$ cannot be $\theta$-admissible. In the classical situation of Theorem BM1 there are no such "minimal" and positive majorants: if $\theta=e^{i \sigma z}, \sigma>0$, a majorant $\omega$ is $\theta$ admissible, and $\omega(x)>0$ for all $x \in \mathbb{R}$, then the majorant $\omega /(1+|x|)$ is also $\theta$-admissible; see [HMII, p. 1265]. 
The structure of the class $\operatorname{Adm} \theta$ depends critically on the velocity of rotation of the unit vector $\theta(x)$ as $x$ increases along $\mathbb{R}$. If $\theta$ is meromorphic (we shall restrict ourselves precisely to this case in what follows), then the unimodular function $\theta(x)$ is analytic on $\mathbb{R}$, so that

$$
\theta(x)=e^{i \varphi(x)}, \quad x \in \mathbb{R},
$$

where $\varphi$ is a monotone increasing real function analytic on $\mathbb{R}$ and determined uniquely up to a constant summand, which is an integral multiple of $2 \pi$. We shall denote $\varphi$ by $\arg \theta$. The $\theta$-admissibility criteria for a majorant $\omega$ obtained in [HMI, HMII are quite different in nature, depending on the behavior of the quantity $(\arg \theta)^{\prime}(x)$ for large $|x|$. In [HMI], mainly the cases where $(\arg \theta)^{\prime}(x)=o(1)$ as $|x| \rightarrow \infty$ were treated (see also Subsection 3.5.3). They fall far apart from the classical situation $\left(\theta(z)=e^{i \sigma z}, \sigma>0\right)$ and resemble the case of a finite Blaschke product $\theta$ mentioned above: there is a "minimal" positive majorant in $\operatorname{Adm} \theta$, and often it can be expressed in terms of the zeros of the Blaschke product $\theta$ (a factor of the form $e^{i \sigma z}$ is absent in this case). Results of this kind will be discussed in Subsection 3.5.2. The situation is close to that of Theorem BM1 for meromorphic functions $\theta$ with the speed $(\arg \theta)^{\prime}(x)$ bounded away from zero or even growing indefinitely. Such functions will be considered in Subsection 3.5.4.

3.5.1. Let $\omega_{1}$ and $\omega_{2}$ be majorants. If $\omega_{1} \leq C \omega$, where $C \geq 0$, we write $\omega_{1} \prec \omega_{2}$. Next, we say that $\omega_{1}$ and $\omega_{2}$ are comparable and write $\omega_{1} \asymp \omega_{2}$ if $\omega_{1} \prec \omega_{2}$ and $\omega_{2} \prec \omega_{1}$. A majorant $\omega$ is said to be $\theta$-minimal if $\omega \in \operatorname{Adm} \theta$ and

$$
\omega_{1} \in \operatorname{Adm} \theta, \omega_{1} \prec \omega \Longrightarrow \omega \asymp \omega_{1} .
$$

We say that a $\theta$-minimal majorant $\omega$ is unique if every $\theta$-minimal majorant is comparable with $\omega$.

Observe that a $\theta$-minimal and positive majorant $\omega$ possesses the following property: every function $f \in K_{\theta}$ subordinate to $\omega$ and such that

$$
\lim _{x \rightarrow+\infty}|f(x)| / \omega(x)=0
$$

is zero (clearly, $+\infty$ can be replaced by $-\infty$ ). In other words, if a function $f \in K_{\theta}$ is subordinate to a minimal and positive majorant $\omega$, then either $|f| \asymp \omega$, or $f \equiv 0$ (recall that if $f \not \equiv 0$ and $f \in K_{\theta}$, then $\left.|f| \in \operatorname{Adm} \theta\right)$.

3.5.2. Relationship with the de Branges spaces. We need a lemma about meromorphic inner functions.

Lemma. The following statements are equivalent: (a) $\theta$ is a meromorphic inner function; (b) there exists an entire function $E$ such that $\left|E^{*}(\zeta)\right|<|E(\zeta)|$ for every $\zeta \in \mathbb{C}_{+}$(where $\left.E^{*}(\zeta):=\overline{E(\bar{\zeta})}\right), E$ does not vanish in $\mathbb{C}_{+} \cup \mathbb{R}$, and

$$
\theta=E^{*} / E \text {. }
$$

This lemma is a consequence of M. G. Krel̆n's theorem about entire functions in the Hermite-Biehler class (see $[\mathrm{Lv}$ ); a direct proof can be found in $\mathrm{HMI}$, pp. 12371238]. The representation (3.2) is obvious if $\theta=B e^{i \sigma z}, \sigma \geq 0$, and the zeros $z_{k}$ of the Blaschke product $B$ are such that $\sum 1 /\left|z_{k}\right|<+\infty$. In this case we can put $E=$ $e^{-i \sigma z / 2} \prod_{k}\left(1-z / \bar{z}_{k}\right)$; if $\sigma=0$, then this function is of zero degree.

With every function $E$ satisfying the conditions of the lemma, we associate the de Branges Hilbert space $\mathcal{H}(E):=\left\{f: f\right.$ is entire, $(f / E) \mid \mathbb{C}_{+}$and $\left(f^{*} / E\right) \mid \mathbb{C}_{+}$belong to $\left.H^{2}\left(\mathbb{C}_{+}\right)\right\}$with the norm $f \mapsto\|f / E\|_{L^{2}(\mathbb{R})}$. The de Branges spaces have numerous applications in function theory and mathematical physics (see DeB, DyM, Rem]).

The space $\mathcal{H}(E)$ is akin to the model subspace $K_{\theta}$, where $\theta=E^{*} / E$. Namely, the operator $f \mapsto f / E$ maps $\mathcal{H}(E)$ onto $K_{\theta}$ isometrically. 
We return to admissible majorants. Among other things, the following dichotomy was established in [HMI, pp. 1245-1246].

Suppose $\theta=E^{*} / E$, where $E$ is an entire function without zeros in $\mathbb{C}_{+} \cup \mathbb{R}$. If $1 / E \in$ $L^{2}(\mathbb{R})$, then $1 / E \in K_{\theta}$, and $(1 /|E|) \mid \mathbb{R}$ is a unique positive and minimal $\theta$-majorant. If $1 / E \notin L^{2}(\mathbb{R})$, then there is no miming positive majorant in $\operatorname{Adm} \theta$; moreover, in this case $\omega /(1+|x|)$ belongs to $\operatorname{Adm} \theta$ whenever $\omega$ is a positive $\theta$-majorant.

To be more precise, the implication $1 / E \in L^{2}(\mathbb{R}) \Longrightarrow 1 / E \in K_{\theta}$ was proved in [HMI] under the following additional assumption on the zeros $z_{n}$ of $E$ :

$$
\sum_{n}\left|z_{n}\right|^{-1}|\log | z_{n}||<+\infty \text {. }
$$

This restriction was lifted in $[\mathrm{BAR}]$ and $[\mathrm{BH}]$.

The square integrability of $1 /|E|$ on $\mathbb{R}$ plays an important role in other questions related to the de Branges spaces $\mathcal{H}(E)$; see Wor, BAR.

3.5.3. "Vertical zeros" of the generating Blaschke product. The above statements reduce an efficient description of the $\operatorname{set} \operatorname{Adm} \theta$ to estimates of the function $|E|$ on the real axis. The case where all zeros of the Blaschke product $\theta=B$ are pure imaginary ("situated vertically") is particularly simple. The Blaschke condition $\sum \operatorname{Im} z_{k} /\left|z_{k}\right|^{2}<\infty$ turns then into $\sum 1 /\left|z_{k}\right|<+\infty$, and the relation $1 / E \in K_{B}$ is fulfilled automatically (see [HMI, p. 1251]). Therefore, $(1 /|E|) \mid \mathbb{R}$ is a unique positive and minimal $B$-majorant. If the sequence $\left(z_{k}\right)$ is sufficiently regular, it is possible to obtain quite a sharp estimate for this majorant, which yields an exhaustive description of the entire set $\operatorname{Adm} B$. For instance, so is the case where $z_{k}=i k^{p}, k=1,2, \ldots$, and $p>1$ (see [HMI, p. 1252]). Here we restrict ourselves to the case of $p=2$, when it is very easy to find the asymptotics of $|E(x)|$ as $|x| \rightarrow \infty:$

$$
|E(x)| \sim \frac{1}{2 \pi \sqrt{|x|}} \exp \left(\pi \sqrt{\frac{x}{2}}\right), \quad|x| \rightarrow \infty .
$$

The results stated above show that

$$
\omega_{1 / 2}(x):=\sqrt{1+|x|} \exp (-\pi \sqrt{|x| / 2}) \in \operatorname{Adm} B,
$$

but

$$
\omega_{\varepsilon}(x)=(1+|x|)^{\varepsilon} \exp (-\pi \sqrt{|x| / 2}) \notin \operatorname{Adm} B
$$

for every $\varepsilon \in(0,1 / 2)$. In particular, we see that the usual condition of convergence for the logarithmic integral $\mathcal{L}(\Omega)=\int_{\mathbb{R}}\left(|\log \omega(x)| /\left(1+x^{2}\right)\right) d x$ (satisfied by the very regular majorants $\omega_{\varepsilon}$ with a large excess) is very far from $B$-admissibility. This is related to the fact that the singularities (poles) of the functions belonging to $K_{B}$ (i.e., the points $-i \operatorname{Im} z_{k}, k=1,2, \ldots$ ) are "very far" from $\mathbb{R}$ (more precisely, "approach" $\mathbb{R}$ too slowly at infinity). Therefore, "reserves of analyticity" for a nonzero function $f \in K_{B}$ suffice to ban the estimate $|f| \prec \omega_{\varepsilon}$ even for not very small majorants $\omega_{\varepsilon}$ (with $\varepsilon \in(0,1 / 2)$ ).

As we shall see, generally speaking, a "horizontal" distribution of the zeros of $B$ (i.e., of the poles for $\left.f \in K_{B}\right)$ brings the necessary admissibility condition $\mathcal{L}(\Omega)<\infty$ for $\omega$ closer to being sufficient, and the main interest lies in the "gap" between necessity and sufficiency — as in Theorem BM1. Furthermore, a sufficient condition becomes sooner "qualitative" than "quantitative", forbidding an excessive oscillation of the majorant.

Returning to the case of pure imaginary zeros $z_{k}$, we emphasize that precisely their "vertical character" is important, i.e., their position on a vertical line $\operatorname{Re} z=c$, where, surely, $c$ may differ from 0 . Curiously, if the zeros $z_{k}$ lie on a "slanting" line $\operatorname{Im} z=k \operatorname{Re} z$ (with $k \in \mathbb{R}$ of an arbitrary large absolute value), then the description of $\operatorname{Adm} B$ changes drastically compared to the "vertical" case. In particular, the function $1 / E$ may fall out of $L^{2}(\mathbb{R})$ (see examples in [HMI]). In [BAR, Baranov found a description of the domains 
$G \subset \mathbb{C}_{+}$that include the imaginary semiaxis and have the property that if the zeros $z_{k}$ remain in $G$, then results in the spirit of our "theorem about vertical zeros" remain true (the Stolz angles $\{\operatorname{Im} z>k|\operatorname{Re} z|\}$ are not among such domains).

3.5.4. Fast rotation of the unit vector $\theta(x)$. Here we again invoke the approach applied in $\S 1$ for the study of $e^{i \sigma x}$-admissibility. We resort to Dyakonov's description of the moduli of the functions in the model subspace $K_{\theta}$; see [DK. This description applies to an arbitrary (not necessarily meromorphic) inner function $\theta$.

Theorem (see [DK]). Suppose $\lambda$ is a nonnegative function in $L^{2}(\mathbb{R})$ and $\theta$ is an inner function. The following two conditions are equivalent: (a) $\lambda=|f|$, where $f \in K_{\theta}$; (b) $\lambda^{2} \theta \in H^{1}(\mathbb{R})$.

If (a) is fulfilled, the function $f$ can be assumed to be outer. The above statement is a partial case of Dyakonov's result about $L^{p}$-analogs of the spaces $K_{\theta}$, which contains some additional information. The content of Subsection 1.9 corresponds to $\theta=e^{i \sigma x}$, $\sigma>0$.

The $\theta$-admissibility of a majorant $\omega$ means the existence of a nonzero nonnegative function $m \in L^{\infty}(\mathbb{R})$ such that $\lambda:=m \omega$ belongs to $L^{2}(\mathbb{R})$ and possesses property (a) in Dyakonov's theorem. In other words, $\omega$ is $\theta$-admissible if and only if there exists a nonnegative function $m \in L^{\infty}(\mathbb{R})$ such that $m \omega \neq 0$ and $m^{2} \omega^{2} \theta \in H^{1}(\mathbb{R})$.

The last membership relation can be rewritten as follows:

$$
m^{2} \omega^{2} \theta=O I
$$

where $I$ is an inner function and $O$ is an outer function in $H^{1}(\mathbb{R})$. If $\theta$ is meromorphic and $I=1$, then (3.3) takes the form

$$
m^{2} \omega^{2} \theta=m^{2} \omega^{2} e^{i(\widetilde{\log m}+2 \widetilde{\log \omega})} ;
$$

that is,

$$
\arg \theta+2 \widetilde{\Omega}=2 \widetilde{\log m}+2 k \pi
$$

where $k$ is a function on $\mathbb{R}$ with values in the set of integers. However, the solvability of equation (3.4) with unknown functions $m$ and $k$, where $m \geq 0, m \omega \in L^{2}(\mathbb{R}), \log m \in$ $L^{1}(P)$, and the values of $k$ are integers, is not merely necessary but also sufficient for the $\theta$-admissibility of $\omega$, no matter what an outer and not necessarily meromorphic function $\omega$ may be, if $\arg \theta$ stands for an arbitrary (possibly, discontinuous) real measurable function with $\exp i \arg \theta=\theta$ a.e. on $\mathbb{R}$ (for the necessity of condition (3.4), see $[\mathrm{BH}$ ], where it was shown that the assumption $I=1$ does not lead to any loss of generality).

Some solvability conditions for equation (3.4) in the situation of Beurling and Malliavin were discussed in $\S 1$. They are well adjusted to functions $\theta$ with "nearly constant" velocity of rotation of the unit vector $\theta(x)$, i.e., to the case where $(\arg \theta)^{\prime}(x) \asymp 1$ as $|x| \rightarrow \infty$. Apart from the classical setting of Beurling and Malliavin, where rotation is uniform $\left((\arg \theta)^{\prime} \equiv \sigma>0\right)$, this case covers Blaschke products $\theta=B$ with nearly horizontal and uniformly distributed zeros. This situation is opposite to that of "vertical zeros" in Subsection 3.5.3. Specifically, it is required that the zeros of $B$ lie in the horizontal strip $\{0<c<\operatorname{Im} z<C\}$ and for some $N, L>0$ any rectangle of the form $(a, a+L) \times(c, C)$, where $a \in \mathbb{R}$, contains at least 1 and at most $N$ zeros of $B$. Under these assumptions, the $B$-admissibility of a majorant $\omega$ can be established in the same way as in the proof of Theorem BM1 in $\S \S 1,2$, provided $\Omega \in \operatorname{Lip}_{1}(\mathbb{R})$; moreover, a nonzero (outer) function $f \in K_{B}$ subordinate to $\omega$ is given by an explicit formula. It should be noted that for the Blaschke products $B$ of the above form, the inclusions $\operatorname{Adm} e^{i \sigma_{1} x} \subset \operatorname{Adm} B \subset \operatorname{Adm} e^{i \sigma_{2} x}$ for some $\sigma_{1}, \sigma_{2}$ can be deduced from Theorem 1 about $\sigma$-admissibility in Subsection 1.14. 
In the general case (i.e., for an arbitrary meromorphic Blaschke product $\theta=B$ ), the angular velocity $(\arg \theta)^{\prime}$ may differ drastically at different portions of $\mathbb{R}$ (in particular, it can be very large somewhere). In order to study $B$-admissibility for fairly wide classes of Blaschke products $B$, it is useful to have a sufficient condition for the solvability of (3.4). We shall state this condition after the following definition.

Definition. Let $\varphi$ be a real function of class $C^{1}(\mathbb{R})$. Suppose there exists a strictly monotone increasing sequence $\left(d_{n}\right)_{n \in A}$ of reals $\left(A\right.$ is either $\mathbb{Z}$ or $\mathbb{N}$ ) such that $\lim _{n \rightarrow+\infty} d_{n}=$ $+\infty, \lim _{n \rightarrow-\infty} d_{n}=-\infty$ (the latter is for $A=\mathbb{Z}$; if $A=\mathbb{N}$, we put $d_{1}=-\infty$ ), and the oscillations of $\varphi$ and $\varphi^{\prime}$ on the intervals $I_{n}=\left(d_{n}, d_{n+1}\right)$ are bounded uniformly in $n$ :

$$
\operatorname{osc}_{I_{n}} \varphi+\operatorname{osc}_{I_{n}} \varphi^{\prime} \leq C
$$

for some $C>0$ and all $n \in A$; moreover, suppose that

$$
a \leq \varphi\left(d_{n+1}\right)-\varphi\left(d_{n}\right) \leq b
$$

for some $a, b>0$ and all $n \in A$. Then $\varphi$ is said to be mainly increasing.

The oscillation of a function $\psi$ on a set $I \subset \mathbb{R}$ is defined to be the quantity $\operatorname{osc}_{I} \psi:=$ $\sup \left\{\left|\psi\left(x^{\prime}\right)-\psi\left(x^{\prime \prime}\right)\right|: x^{\prime}, x^{\prime \prime} \in I\right\}$.

A mainly increasing function $\varphi$ is indeed strictly monotone increasing along the sequence $\left(d_{n}\right)_{n \in A}$; between adjacent points $d_{n}$, i.e., on the intervals $I_{n}$, it may fail to be monotone increasing, but $\varphi$ remains "level" on these intervals uniformly in $n$.

Theorem. If $\varphi$ is a mainly increasing function, then $\varphi=\widetilde{2 \log m}+2 k \pi$, where $k$ is a function whose values are integers, and $m \in L^{\infty}(\mathbb{R}) \cap L^{2}(\mathbb{R}), m \geq 0, \log m \in L^{1}(P)$.

This theorem remains true if we relax the condition for a function to be mainly increasing: the requirement (3.5) may be replaced by

$$
\operatorname{osc}_{I_{n}} \varphi+\frac{1}{\left|I_{n}\right|} \sup _{x \in I_{n}} \int_{I_{n}}\left|\varphi^{\prime}(x)-\varphi^{\prime}(t)\right| d t \leq C
$$

furthermore, we can remove the smoothness assumption for $\varphi$, replacing it with the condition of absolute continuity.

Condition (3.5) (and also (3.6)) is fulfilled if the lengths $\left|I_{n}\right|$ are uniformly bounded and $\varphi \in \operatorname{Lip}_{1}(\mathbb{R})$ (this is so if $\varphi=\sigma x+2 \widetilde{\Omega}$ whenever $\widetilde{\Omega} \in \operatorname{Lip}_{1}(\mathbb{R}),\left\|\widetilde{\Omega}^{\prime}\right\|_{\infty}<\sigma / 2$, and $\left.\varphi\left(d_{n}\right)=2 \pi n, n \in \mathbb{Z}\right)$.

A slightly weaker version of the last theorem was proved in [HMII]: there the additional assumption $\sum_{n}\left|I_{n}\right|^{2} /\left(d_{n} d_{n+1}\right)<+\infty$ was imposed (in particular, this is so if $\sup _{n}\left|I_{n}\right|<$ $\infty$; in the general case, we only have $\left.\sum_{n}\left|I_{n}\right| /\left(d_{n} d_{n+1}\right)<+\infty\right)$. In the wording given here, the theorem was proved in [BH]. The version in [HMII] made it possible to obtain a certain condition sufficient for $B_{\alpha}$-admissibility in the case of Blaschke products $B_{\alpha}$ with "horizontal" zeros of the form $|n|^{\alpha} \operatorname{sgn} n+i, n \in \mathbb{Z}$, where $\alpha \in(1 / 2,1)$. The restriction $\alpha>1 / 2$ is dictated by the Blaschke condition. The case of $\alpha=1$ is very close to the classical situation of Beurling and Malliavin. But if $\alpha>1$, we are in the situation of Subsection 5.3, where a minimal positive $B_{\alpha}$-majorant can be exhibited. If $\alpha \in(1 / 2,1)$, then $\left(\arg B_{\alpha}\right)^{\prime}(x) \asymp|x|^{1 / \alpha-1}$ as $|x| \rightarrow \infty$. Conditions sufficient for $B_{\alpha}$-admissibility that were described in [HMII] allow some growth of $\left|\widetilde{\Omega}^{\prime}(x)\right|$ as $|x| \rightarrow \infty$; they become weaker as $\alpha$ approaches $1 / 2$. By using the techniques of our $\S 2$, Yu. S. Belov was able to find $B_{\alpha}$-admissibility conditions imposed directly on $\Omega$ rather than on $\widetilde{\Omega}$.

Theorems on the representability of almost increasing functions in the form $\widetilde{2 \log m}+$ $2 k \pi$ played a prominent role in the paper $\mathrm{BBH}$, containing a detailed study of admissible majorants for model subspaces generated by meromorphic Blaschke products with zeros distributed regularly in a strip or a half-strip. In particular, in $\mathrm{BBH}$ the Blaschke 
product with "one-sided" zeros $n^{\alpha}+i, n \in \mathbb{N}, \alpha>1 / 2$, was considered, and also the Blaschke product with zeros $n+i y_{n}$, where $n \in \mathbb{N}$ and $y_{n} \rightarrow 0$ (the case of a tangential approach to the real line). Besides the admissibility criteria described above, some quasianalyticity or weighted polynomial approximation results prove to be quite useful in such problems.

We have already considered some conditions for the existence of a minimal positive majorant. However, if we renounce positivity, minimal majorants always exist; see $[B \mathrm{BH}$. First, we present a result of [HMI, p. 1260], which is applicable to arbitrary (rather than merely to meromorphic) inner functions. An inner function $\Theta$ is said to be the circular part of a function $g \in H^{1}$ if $g(x)=\Theta(x)|g(x)|$ for a.e. $x \in \mathbb{R}$.

Theorem. If $\Theta$ is the circular part of some outer function $g \in H^{1}$, then the function $w=\sqrt{|g|}$ is a minimal admissible majorant for $K_{\Theta}$.

It turns out that the condition " $\Theta$ is the circular part of an outer $H^{1}$-function" imposes no restrictions on $\Theta$. It was shown in $[\mathrm{BH}]$ that every inner function is the circular part of some outer function $g$ in $H^{1}$, and thus, any model subspace $K_{\Theta}$ possesses minimal majorants. Such majorants may have real zeros and are not necessarily unique. For instance, if $\Theta(z)=e^{i \sigma z}$, then $w(t)=\left|\frac{\sin \sigma t}{t}\right|$ is a minimal $e^{i \sigma z}$-majorant.

3.6. Details of proofs skipped in $\S \S 1,2$; comments. Useful properties of the Hilbert transformation, and also some simple calculations are described in the first four subsections, written to save the reader's time and to spare him addressing special manuals. With the same purpose, in Subsection 3.6.5 a "purely real" proof of the positivity of the spectrum of an outer function is presented (it was only sketched in $\$ 1$ ). Subsection 3.6.6 contains a complex analysis argument yielding the main "admissibility equation" of Subsection 1.10. The last three subsections contain comments on the proof of Theorem 2 of Subsection 2.1; in Subsection 3.6.9 it is shown how the result of Subsection 1.16 (on the admissibility of a majorant monotone decreasing on the rays $(-\infty, 0)$ and $(0,+\infty))$ can be obtained by the methods of $\S 2$ (surely, no dyadic microanalysis is required this time).

3.6.1. On the Hilbert transformation. The following ("complex") definition of the operator $h$ is recognized as conventional: a real function $f$ on $\mathbb{R}$ gives rise to its harmonic extension to the upper half-plane $\mathbb{C}_{+}$, i.e., the solution $u$ of the Dirichlet problem for $\mathbb{C}_{+}$ expressed via the Poisson integral,

$$
u(x+i y)=\frac{y}{\pi} \int_{-\infty}^{+\infty} f(t) /\left((x-t)^{2}+y^{2}\right) d t, \quad x \in \mathbb{R}, \quad y>0
$$

(so, we forcedly assume that $f \in L^{1}(P)$ ). After that we find a real function $v$ on $\mathbb{C}_{+}$such that $u+i v$ is analytic in $\mathbb{C}_{+}$and $v(i)=0$. It can be shown that $\lim _{s \downarrow 0} v(t+i s)=h(f)(t)$ for $m$-a.e. $t \in \mathbb{R}$; see $[\mathrm{Ko04}$.

This interpretation of the Hilbert transformation simplifies and clarifies many calculations, but we did not resort to it in $\S \S 1,2$ because we wished to give an entirely real-variable proof of Theorem BM1.

It should be noted that we require the operator $h$ with improved Cauchy kernel rather than $h_{0}$ (with the "pure" Cauchy kernel) because the Hilbert transformation is applied mostly to $\Omega$, and this function lies in $L^{1}(P)$ but not in $L^{p}(m)$ with some $1 \leq p \leq+\infty$, where $m$ is Lebesgue measure.

3.6.2. Inversion formula for $h$. First, we prove that

$$
\|h(f)\|_{L^{2}(P)} \leq A\|f\|_{L^{2}(P)}, \quad f \in L^{2}(P)
$$

( $A, A_{1}, \ldots$ are universal constants). 
We start with establishing (3.7) for the $f$ that vanish on $\mathbb{R} \backslash[-2,2]$ and then pass to the $f$ that vanish on $[-2,2]$.

1) If $f \equiv 0$ off $[-2,2]$, then, denoting by $m$ Lebesgue measure on $\mathbb{R}$, we obtain

$$
\begin{aligned}
\|h(f)\|_{L^{2}(P)} & \leq\left\|h_{0}(f)\right\|_{L^{2}(P)}+\left|\int_{-2}^{2} f(t) t d P(t)\right| \\
& \leq\left\|h_{0}(f)\right\|_{L^{2}(m)}+2 \int_{-2}^{2}|f| d m \leq A_{0}\|f\|_{L^{2}(m)} \leq A_{1}\|f\|_{L^{2}(P)}
\end{aligned}
$$

(we have used the relations $\left\|h_{0}(f)\right\|_{L^{2}(m)}=\|f\|_{L^{2}(m)}$ and $d P \asymp d m$ on $[-2,2]$ ).

2) If $f \equiv 0$ on $[-2,2]$, then, clearly, $|x-t| \geq|t| / 2$ if $|x|<1$ and $|t|>2$; therefore,

$$
\begin{aligned}
|h(f)(x)| & \leq \int_{|t|>2}|f(t)| \frac{1+|t||x|}{|x-t|\left(t^{2}+1\right)} d t \\
& \leq 2 \int_{|t|>2} \frac{|f(t)|}{t^{2}} d t \leq 20 \int_{\mathbb{R}}|f| d P \leq A_{1}\|f\|_{L^{2}(P)} .
\end{aligned}
$$

Consequently, we have $\int_{-1}^{1}(h(f))^{2} d P \leq A_{2}\|f\|_{L^{2}(P)}^{2}$. Now it remains to estimate $\int_{|x|>1}(h(f))^{2}(x) d P(x)$. Putting $g(t)=f(t) / t$ (so that $\left.g \in L^{2}(m)\right)$, we obtain

$$
\begin{aligned}
h(f)(x) & =x h_{0}(g)(x)-\int_{\mathbb{R}} g d P, \\
\int_{\mathbb{R}}(h(f))^{2} d P & \leq 2 \int x^{2}\left(h_{0}(g)\right)^{2} d P+2\left(\int g d P\right)^{2} \\
& \leq \int_{\mathbb{R}}\left(h_{0}(g)\right)^{2} d m+2 \int_{\mathbb{R} \backslash[-2,2]} g^{2} d P \\
& =\int_{\mathbb{R} \backslash[-2,2]} g^{2} d m+\frac{2}{\pi} \int_{\mathbb{R} \backslash[-2,2]} \frac{(f(x))^{2} d x}{x^{2}\left(1+x^{2}\right)} \\
& \leq A\|f\|_{L^{2}(P)},
\end{aligned}
$$

and (3.7) is proved.

Now, we verify the identity

$$
h(h(f))=-f+\text { const }, \quad f \in L^{2}(P) .
$$

We define $f_{n}=f \chi_{[-n, n]}, n=1,2, \ldots$ For every $n$ we have

$$
h\left(f_{n}\right)=h_{0}\left(f_{n}\right)+c\left(f_{n}\right), \quad c\left(f_{n}\right)=-\int_{\mathbb{R}} f_{n}(x) \frac{x d x}{\pi\left(1+x^{2}\right)},
$$

so that

$$
h\left(h\left(f_{n}\right)\right)=h\left(h_{0}\left(f_{n}\right)\right)=h_{0}\left(h_{0}\left(f_{n}\right)\right)+c\left(h_{0}\left(f_{n}\right)\right)=-f_{n}+c\left(h_{0}\left(f_{n}\right)\right)
$$

(we have used the relation $h$ (const) $=0$ and the identity $h_{0}\left(h_{0}(g)\right) \equiv-g$ for $g \in L^{2}(m)$ ). Next, $f_{n} \rightarrow f$ in $L^{2}(P)$ as $n \rightarrow \infty$ and, by (3.7), $h\left(h\left(f_{n}\right)\right) \underset{n \rightarrow \infty}{\longrightarrow} h(h(f))$. Therefore, the constants $c\left(h\left(f_{n}\right)\right)$ tend to a constant, and, passing to the limit, we obtain (3.8).

3.6.3. The Hilbert transform of the "saw" $s_{\sigma}$. Let $s_{\sigma}$ denote the function with period $1 / \sigma$ that coincides with $\pi \sigma x-\pi / 2$ on $[0,1 / \sigma)$. We show that

$$
h\left(s_{\sigma}\right)(t)=-\log |\sin \pi \sigma t|+C, \quad t / \sigma \notin \mathbb{Z},
$$

where $C$ is a real constant. 
Proof. Since $s_{\sigma}(x)=s_{1 / 2 \pi}(2 \pi \sigma x)$, we may assume that $\sigma=1 / 2 \pi$. On $(0,2 \pi)$, we have

$$
s_{1 / 2 \pi}=(x-\pi) / 2=-\sum_{k=1}^{\infty}(\sin k x) / k,
$$

where the series converges in $L^{2}(0,2 \pi)$ and so it converges to $s_{1 / 2 \pi}$ on the entire line in $L^{2}(P)$, and (see Subsection 3.6.2) $h\left(s_{1 / 2 \pi}\right)=-\sum_{k=1}^{\infty} h(\sin k x) / k$ in $L^{2}(P)$. But $h(\sin k x)=-\cos k x+e^{-k}, k=1,2, \ldots$ This can be shown by direct calculation of the integrals $\int_{\mathbb{R}} e^{i k t}(x-t)^{-1} d t$ and $f_{\mathbb{R}} e^{i k t}\left(t /\left(1+t^{2}\right)\right) d t, k \in \mathbb{Z}$ (the first integral is understood as $\lim _{A \rightarrow+\infty} \int_{-A}^{A}$ and the second as $\left.\lim _{A \rightarrow+\infty} \int_{-A}^{A}\right)$. Thus, $h\left(s_{1 / 2 \pi}\right)=$ $\sum_{k=1}^{\infty} \cos k x / k+C^{\prime}=-\operatorname{Re} \log \left(1-e^{i x}\right)+C^{\prime}=-\log |\sin (x / 2)|+C$, where $C, C^{\prime}$ are real constants; the last formula is valid on $\mathbb{R} \backslash \mathbb{Z}$.

3.6.4. An estimate for $\left\|\tilde{\gamma}^{\prime}\right\|_{\infty}$ (see Subsection 1.13). Putting $\gamma_{k}=\log \left(x^{2}+k^{2}\right)=$ $2 \log k+l(x / k), l=\log \left(1+x^{2}\right)$, we have

$$
\begin{aligned}
& h\left(\gamma_{k}\right)(x)=h(l)(x / k) / k, \\
& h\left(\gamma_{k}\right)^{\prime}(x / k k)=\left[h\left(l^{\prime}\right)(x / k)\right] / k=-2 k /\left(x^{2}+k^{2}\right)+c / k .
\end{aligned}
$$

The last formula follows from the identity $2 x\left(1+x^{2}\right)^{-1}=(x+i)^{-1}+(x-i)^{-1}$ and from the definition of $h$.

3.6.5. The spectrum of an outer function is nonnegative. Here it will be convenient to use the Fourier transformation $T \mapsto \widehat{T}$ on tempered distributions $T \in S^{\prime}(\mathbb{R})=S^{\prime}$ (recall that $\widehat{T}[\alpha]=T[\hat{\alpha}], \alpha \in S$ ). We denote by $S_{+}^{\prime}$ the subspace of $S^{\prime}$ consisting of all distributions $T \in S^{\prime}$ with nonnegative spectrum $\left(T \in S_{+}^{\prime} \Longleftrightarrow \widehat{T}=0\right.$ on $\left.(-\infty, 0)\right)$. Clearly, $S_{+}^{\prime}$ is closed in $S^{\prime}$ with respect to the usual convergence of sequences in $S^{\prime}$.

Suppose $\psi$ is a real function, $\psi \in L^{1}(P), e^{\psi} \in L^{p}(m), 1 \leq p \leq+\infty(P$ and $m$ are Poisson and Lebesgue measures on $\mathbb{R})$. We show that

$$
\text { Ext } \psi \in S_{+}^{\prime} .
$$

Put $\varphi=\psi+i \widetilde{\psi}$, where $\widetilde{\psi}$ is understood as $h(\psi)$. Thus, Ext $\psi=e^{\varphi}$. We split the proof of (3.9) into three parts.

1) Suppose $\psi \in C_{0}^{\infty}(\mathbb{R})$. Then $\varphi \in L^{\infty}(\mathbb{R})$ and $e^{\varphi}=\lim _{n \rightarrow \infty}(1+\varphi / n)^{n}$ uniformly on $\mathbb{R}$. But $\varphi \in S_{+}^{\prime}$, so that $(1+\varphi / n)^{n} \in S_{+}^{\prime}$, and we obtain (3.9).

$2)$ Suppose $\psi \in L^{\infty}(\mathbb{R})$. We construct a sequence $\left(\psi_{n}\right)$ in $C_{0}^{\infty}(\mathbb{R})$ such that $\psi_{n} \rightarrow \psi$ in $L^{2}(P)$ and $\left\|\psi_{n}\right\|_{\infty} \leq c$. Putting $\varphi_{n}=\psi_{n}+i \widetilde{\psi}_{n}$, by 1$)$ we obtain $e^{\varphi_{n}} \in S_{+}^{\prime}$. However, $\varphi_{n} \rightarrow \varphi$ in $L^{2}(P)$ (see the beginning of Subsection 3.6.2). Therefore, $e^{\varphi_{n}} \rightarrow e^{\varphi}$ in $m$ measure and $\left\|e^{\varphi_{n}}\right\|_{\infty}=\left\|e^{\psi_{n}}\right\|_{\infty} \leq e^{c}$. Consequently, $e^{\varphi_{n}} \rightarrow e^{\varphi}$ in $S^{\prime}$, and again we obtain (3.9).

3) In the general case we have $\psi=\psi_{+}-\psi_{-}$, where $\psi_{ \pm}$and $\psi_{-}$are the positive and the negative part of $\psi$. Then $\varphi=\varphi^{+}-\varphi^{-}$, where $\varphi_{ \pm}=\psi_{ \pm}+i \widetilde{\psi}_{ \pm}$, and $e^{\varphi}=e^{\varphi^{+}} e^{-\varphi^{-}}$. It suffices to check that $e^{\varphi^{+}}, e^{-\varphi^{-}} \in S_{+}^{\prime}$ (note that $e^{-\varphi^{-}} \in L^{\infty}(m), e^{\varphi^{+}} \in L^{p}(m)$ ). For this, we consider the upper truncations $\psi_{n}^{ \pm}=\min \left(\varphi^{ \pm}, n\right)$ and put $\varphi_{n}^{ \pm}=\psi_{n}^{ \pm}+i \widetilde{\psi}_{n}^{ \pm}$. We use the fact that $\left|e^{-\varphi_{n}^{-}}\right| \leq 1,\left|e^{\varphi_{n}^{+}}\right| \leq e^{\psi} \in L^{p}(m)$, and $e^{ \pm \varphi_{n}^{ \pm}} \rightarrow e^{\varphi}$ in $m$-measure. The last convergence follows from the Kolmogorov theorem for $p=1$, from the $L^{2}(P)$-continuity of $h$ for $p \in[2,+\infty]$, and from the M. Riesz theorem for $1<p<2$ (see [Koo4; however, such $p$ 's do not occur in $\S \S 1,2)$. So, $e^{\varphi_{n}^{ \pm}} \rightarrow e^{\varphi^{ \pm}}$in $S^{\prime}$, and (3.9) follows from 2). 
3.6.6. A complex analysis argument yielding equation $(*)$ in Subsection 1.10. This equation, which characterizes the admissible majorants, can be deduced by using simple facts of complex analysis (we avoided them in $\S 1$ intentionally). By the PaleyWiener theorem, the set $P W_{\sigma}$ coincides with the traces on $\mathbb{R}$ of the entire functions of degree at most $\sigma$ that are square integrable on $\mathbb{R}$ (for short, here we change the normalization of the Fourier transforms adopted in $\S 1$ and omit the factor $2 \pi$ in the exponential). Therefore, a majorant $\omega$ is $\sigma$-admissible if and only if there exists an entire function $f \not \equiv 0$ such that $|f| \leq \omega$ on $\mathbb{R},|f(z)|=O\left(e^{\sigma|z|}\right)$ as $|z| \rightarrow \infty$, and $\int_{-\infty}^{+\infty}|f(x)|^{2} d x<+\infty$. Mainly, we are interested in full admissibility for $\omega$; therefore, we should not insist on fixing $\sigma$ rigidly. We would be content even with an entire function $f \in B_{\sigma}$, where $B_{\sigma}$ is the set of all (not necessarily square integrable) entire functions of degree at most $\sigma$ and bounded on $\mathbb{R}$ : if $|f| \leq \omega$ on $\mathbb{R}$ and $f \in B_{\sigma}$, then the function $f_{\varepsilon}=(f \sin \varepsilon z) / z$ belongs to $P W_{\sigma_{+} \varepsilon}$ and $\left|f_{\varepsilon}\right| \leq \operatorname{const} \omega$ on $\mathbb{R}$.

Now we observe that, by the Riemann symmetry principle, a nonnegative continuous function $h \in L^{\infty}(\mathbb{R})$ coincides on $\mathbb{R}$ with some function in $B_{\sigma}$ if and only if $h$ can be extended to the set $\mathbb{C}_{+} \cup \mathbb{R}$ up to a function $H$ analytic in $\mathbb{C}_{+}$and satisfying $|H(z)|=$ $O(\exp \sigma|z|)$ as $|z| \rightarrow \infty, z \in \mathbb{C}_{+}$. Next, by the Phragmen-Lindelöf principle, such a function $H$ is characterized by the relation $e^{i \sigma z} H \in H^{\infty}\left(\mathbb{C}_{+}\right)$.

Returning to the majorant $\omega$, we put $h=m \omega$, where $m \in L^{\infty}(\mathbb{R})$ is an unknown nonnegative function to be chosen in such a way that the product $\omega m e^{i \sigma x}$ coincides on $\mathbb{R}$ with the trace of some function continuous on $\mathbb{C}_{+} \cup \mathbb{R}$ and analytic and bounded on $\mathbb{C}_{+}$. Then $\omega m$ coincides on $\mathbb{R}$ with some (nonnegative) function in $B_{\sigma}$ that does not exceed $\|m\|_{\infty} \omega$ on $\mathbb{R}$. We require that $\omega m e^{i \sigma x}$ be outer, i.e.,

$$
\omega m e^{i \sigma x}=\omega m e^{i(\widetilde{\log \omega}+\widetilde{\log m})} .
$$

We get the equation

$$
\widetilde{\log m}+\widetilde{\log \omega}=\sigma x \bmod 2 \pi,
$$

which differs from $(*)$ in Subsection 1.10 only by the absence of the factors 2 and $\pi$; the unknown function $m$ must satisfy the conditions

$$
m \geq 0, \quad \log m \in L^{1}(P), \quad m \in L^{\infty}(\mathbb{R}), \quad m \omega \in L^{2}(\mathbb{R}) .
$$

In the main body of the text, we did not use complex tools and preferred a purely real approach leading to a characterization of arbitrary $\theta$-admissible majorants (see equation (3.4) in Subsection 3.5.4).

3.6.7. Estimate (2.14) and the Hadamard-Landau inequality. We mean the well-known estimate of the first derivative in terms of the uniform norms of the function itself and its second derivative on a bounded interval (see [T, §2.4], [HLP, and MGLP, p. 420]). Indeed, if the function is the primitive $\Phi$ of a nonnegative function $f$ that vanishes at the left endpoint of the interval, then $\|\Phi\|_{\infty}=\int_{I} f,\left\|f^{\prime}\right\|_{\infty}=\left\|\Phi^{\prime \prime}\right\|_{\infty}$, and (2.14) is a partial case of the Hadamard-Landau inequality.

We have used the condition $f \in \operatorname{Lip}_{1}(I)$, as well as the Lipschitz condition for $\Omega$ in Theorem BM1, only for estimating integrals in (2.14) and in Subsection 2.3. In principle, such estimates are possible not only in terms of $\left\|f^{\prime}\right\|_{\infty}$ (as is done here) but also in terms of other seminorms (there is an extensive literature on this subject; see [T, MIT]). This gives us hope that our approach might be applicable to more than just the Lipschitz functions $\Omega$ in Theorem BM1.

3.6.8. Lonely tooth (commentary on the main lemma in $\S 2$ ). Here we discuss two points related to the main lemma in $\S 2$ (see Subsection 2.6). In both cases, we use a very simple Lipschitz function $f=f_{h}$ (a "lonely tooth") depicted in Figure 8. 


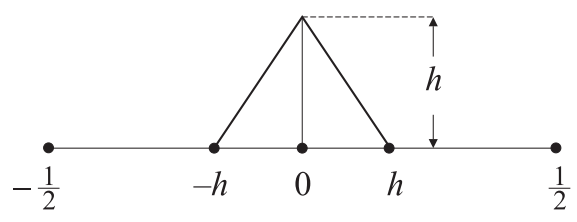

FiguRE 8 .

A) Let $h=\delta / 2$, and suppose that a Lipschitz function $F$ majorizes the tooth $f$, and moreover, $F \equiv 0$ off $(3 / 2) I^{*}$ and $\left\|F^{\prime}\right\|_{\infty} \leq A \delta$. Then, in particular, $F(0) \geq f(0)=\delta / 2$, so that $F(t) \geq(\delta / 2-A \delta|t|)_{+}$for every $t \in \mathbb{R}$. Consequently,

$$
\int_{\mathbb{R}} F \geq \int_{|t| \leq 1 / 2 A}(1 / 2-A|t|) d t=\delta / 4 A,
$$

whereas $\int_{I^{*}} f=\delta^{2} / 4$, and we see that the factor $\delta$ on the right in estimate 5 ) of the main lemma $\left(\int_{I^{*}} f \geq C \delta \int F_{\mathbb{R}}\right)$ cannot be avoided.

B) It might have been possible to try to estimate the quantity $\left\|\widetilde{F}^{\prime}\right\|_{\infty}$ in the main lemma without direct invocation of $\widetilde{F}^{\prime}=h_{0}\left(F^{\prime}\right)$, applying instead some standard trick of estimating a Hilbert transform uniformly. For instance, if (for $\delta=1$ ) the majorant $F$ satisfying conditions 1), 2), 3), and 5) of the main lemma would have admitted an estimate for the second derivative (in place of 4)):

$$
\left\|F^{\prime \prime}\right\|_{\infty} \leq A
$$

or, at the worst, if we could have controlled the Hölder norm for $F^{\prime}$ of some order $\alpha \in(0,1]:$

$$
\sup _{\substack{x, y \in \mathbb{R} \\ x \neq y}}\left|F^{\prime}(x)-F^{\prime}(y)\right| /|x-y|^{\alpha} \leq A,
$$

then we would have obtained estimate 4$)\left(\left\|\widetilde{F}^{\prime}\right\|_{\infty} \leq A\right)$ for free. Indeed, restricting ourselves to the case of $\delta=1, I=I^{*}=[-1 / 2,1 / 2]$, we would have had $\left\|F^{\prime}\right\|_{\infty} \leq A_{2}$ (by condition 3$)$ or by (3.10) and the fact that $F \equiv 0$ off $\left.(3 / 2) I^{*}\right)$, so that for $|x|>1$ the inequality

$$
\left|\widetilde{F}^{\prime}(x)\right| \leq A_{2} \int_{3 / 2 I^{*}} \frac{d t}{|x-t|} \leq A_{3}
$$

would have been true, and for $|x| \leq 1$ we would have deduced from (3.10) that

$$
\begin{aligned}
\left|\widetilde{F}^{\prime}(x)\right| & \leq \int_{-1}^{1} \frac{\left|F^{\prime}(t)-F^{\prime}(x)\right|}{|t-x|} d t+\left|F^{\prime}(x)\right|\left|f_{-1}^{1} \frac{d t}{t-x}\right| \\
& \leq \sup _{|x| \leq 1}\left(A \int_{-1}^{1} \frac{d t}{|t-x|^{1-\alpha}}+\left|F^{\prime}(x) \log \right| \frac{1-x}{1+x}||\right)=A_{4} .
\end{aligned}
$$

However, it is impossible to bypass direct estimation of $\left\|\widetilde{F}^{\prime}\right\|_{\infty}$ by replacing it with (3.10). Again, a counterexample is given by the function $f_{h}=(h-|x|)_{+}$(Figure 8).

Suppose for some $\alpha \in(0,1]$ and every $h \in(0,1 / 2]$ we can find a majorant $F=F_{h}$ of the tooth $f_{h}$ satisfying 1)-3) and 5) of the main lemma $\left(\delta=1, I=I^{*}\right)$, but with 4) replaced by (3.10). Clearly, $\int_{I^{*}} f=h^{2}, \int_{\mathbb{R}} F \leq A \int_{I^{*}} f=A h^{2}$. We fix a number $\lambda \in(0,1)$ very close to 1 (the choice of $\lambda$ will be specified at the end of the argument). Averaging $F$ over the interval $(0, h)$ (which is "long" compared to $\left(0, h^{\lambda}\right)$ ), we obtain

$$
F(c)=h^{-\lambda} \int_{0}^{h^{\lambda}} F \leq A h^{2-\lambda}
$$


where $c \in\left(0, h^{\lambda}\right)$. But $F(0) \geq f(0)=h$, so that the oscillation of $F$ on $\left[0, h^{\lambda}\right]$ is at least $h-A h^{2-\lambda} \geq h / 2$ if $0<h<h(A, \lambda)$ (recall that $2-\lambda>1$ ). Consequently, $F^{\prime}\left(\theta_{+}\right) \cdot h^{\lambda} \geq h / 2$ for some $\theta_{+} \in\left(0, h^{\lambda}\right)$, and $F^{\prime}\left(\theta_{+}\right) \geq h^{1-\lambda} / 2$. In the same way, we find a point $\theta_{-} \in\left(-h^{\lambda}, 0\right)$ such that $F^{\prime}\left(\theta_{-}\right) \leq h^{1-\lambda} / 2$. But (3.10) implies

$$
(2 h)^{\alpha \lambda} A \geq A\left(\theta_{+}-\theta_{-}\right)^{\alpha} \geq F^{\prime}\left(\theta_{+}\right)-F^{\prime}\left(\theta_{-}\right) \geq h^{1-\lambda}
$$

for arbitrarily small $h>0$. Taking $\lambda$ with $1-\lambda<\alpha \lambda$, we arrive at a contradiction.

For a majorant $F_{h}$ of $f_{h}$ with the required properties (i.e., properties 1)-5)), we can take the cap $\varphi_{[-3 / 2 h, 3 / 2 h]}^{*}$ adjusted to the interval $[-3 / 2 h, 3 / 2 h]$ (see Subsection 2.5.1). However, $\left\|F_{h}^{\prime \prime}\right\| \asymp 1 / h$; as $h \rightarrow 0$, the Hölder norm of $F_{h}^{\prime}$ (of any order $\left.\alpha \in(0,1]\right)$ also grows unboundedly.

3.6.9. Another proof of the theorem in Subsection 1.16 (an even majorant monotone decreasing on the ray $(0,+\infty)$. As in Subsection 1.16, we assume that $\Omega$ is even and monotone increasing on the ray $(0,+\infty)$, and $\mathcal{L}(\Omega)<+\infty$. We also assume that $\Omega \equiv 0$ on $[-2,2]$ and return to Subsection 2.4 , keeping the notation introduced there. Observe that this time

$$
\int_{\mathbb{R}} \frac{\Omega(x)}{x^{2}} d x \asymp \sum_{j=1}^{\infty} \frac{\Omega\left(2^{j}\right)}{2^{j}}
$$

(with universal constants), and for $x>0$ we have

$$
\Omega(x) \leq x \int_{x}^{\infty} \frac{\Omega(t)}{t^{2}} d t \leq x \int_{0}^{\infty} \frac{\Omega(t)}{t^{2}} d t
$$

so that also the quantity $S=\sup \Omega(x) /|x|$ can be assumed as small as we wish. We put $\Omega_{1, j}=\Omega\left(2^{j+1}\right) 2^{-j} \varphi_{I_{j}}$, where $\varphi$ is the standard cap and $\varphi_{I_{j}}$ is its adjustment to $I_{j}$; here $j \geq 1$. For $j \leq-1$ we take $\Omega_{1, j}(x)=\Omega_{1,|j|}(|x|)$, and $\Omega_{1,0} \equiv 0$. Then $\Omega_{1}=\sum_{j} \Omega_{1, j} \geq \Omega$. Next, $\left\|\Omega_{1, j}\right\|_{\infty} \leq \Omega\left(2^{j+1}\right)$, whence

$$
\int_{\mathbb{R}} \frac{\Omega_{1}(x)}{x^{2}} d x \leq A \int \frac{\Omega(x)}{x^{2}} d x,
$$

and also the integral $\int_{\mathbb{R}} \frac{\Omega_{1}(x)}{x^{2}} d x$ can be made as small as we wish. The proof is finished as in Subsection 2.4. We only dwell on the most problematic estimates (2.9). This time, directly from the definition of the "pieces" $\Omega_{1, j}$, we find

$$
\left\|\widetilde{\Omega}_{1, j}^{\prime}\right\|_{\infty} \leq \frac{\Omega\left(2^{j+1}\right)}{2^{j+1}}\left\|\widetilde{\varphi}^{\prime}\right\|_{\infty} \leq S\|\widetilde{\varphi}\|_{\infty} ;
$$

again, the last product is arbitrarily small. Thus, if $\omega$ does not oscillate at all, it is possible to get by only with the initial stage of the proof of Theorem 2, not penetrating deeply into long intervals $I_{j}$ and avoiding the dyadic microanalysis, which was discussed in a large segment of $\S 2$.

Acknowledgements. P. Koosis helped us to shorten the initial proof of inequality (2.24) in Subsection 2.7.1; M. S. Birman and S. V. Kislyakov made many helpful critical remarks at the final stage of editing; A. D. Baranov took part in writing Subsection 3.5. We express our sincere gratitude to all of them.

\section{REFERENCES}

[BAR] A. D. Baranov, Polynomials in the de Branges space of entire functions, Ark. Mat. (to appear).

$[\mathrm{BBH}]$ A. D. Baranov, A. A. Borichev, and V. P. Havin, Admissible majorants for meromorphic functions with fixed poles (in preparation).

[BE] A. Beurling, On two problems concerning linear transformations in Hilbert space, Acta Math. 81 (1948), 17 pp. MR0027954(10:381e) 
[BH] A. D. Baranov and V. P. Havin, Admissible majorants for model subspaces and arguments of inner functions (in preparation).

[BK] K. Barbey and H. König, Abstract analytic function theory and Hardy algebras, Lecture Notes in Math., vol. 593, Springer-Verlag, Berlin-New York, 1977. MR0442690 (56:1071)

[BM1] A. Beurling and P. Malliavin, On Fourier transforms of measures with compact support, Acta Math. 107 (1962), 291-309. MR0147848 (26:5361)

[BM2] - On the closure of characters and the zeros of entire functions, Acta Math. 118 (1967), 79-93. MR0209758(35:654)

[BeH] Yu. S. Belov and V. P. Khavin, On a theorem of I. I. Privalov on the Hilbert transform of Lipschitz functions, Mat. Fiz. Anal. Geom. 11 (2004), no. 4, 380-407. (Russian) MR2114001 (2005k:26006)

[Bu] M. A. Bulgakov, White guard. Novels. The Master and Margarita, Lenizdat, Leningrad, 1989. (Russian)

[DK] K. M. D'yakonov, Moduli and arguments of analytic functions from subspaces in $H^{p}$ that are invariant under the backward shift operator, Sibirsk. Mat. Zh. 31 (1990), no. 6, 64-79; English transl., Siberian Math. J. 31 (1990), no. 6, 926-939 (1991). MR1097956 (92f:30049)

[D] P. Duren, Theory of $H^{p}$ spaces, Pure and Appl. Math., vol. 38, Acad. Press, New York-London, 1970. MR0268655 (42:3552)

[DeB] L. de Branges, Hilbert spaces of entire functions, Prentice-Hall, Inc., Englewood Cliffs, NJ, 1968. MR0229011 (37:4590)

[DyM] H. Dym and H. P. McKean, Gaussian processes, function theory, and the inverse spectral problem, Probab. Math. Statist., vol. 31, Acad. Press, New York-London, 1976. MR0448523 (56:6829)

[GLO] A. A. Gol'dberg, B. Ya. Levin, and I. V. Ostrovskǐ̌, Entire and meromorphic functions, Complex Analysis. One Variable, 1, Itogi Nauki i Tekhniki Sovrem. Probl. Mat. Fund. Naprav., vol. 85, VINITI, Moscow, 1991, pp. 5-185; English transl., Encyclopaedia Math. Sci., vol. 85, Springer, Berlin, 1997, pp. 1-193. MR.1155417 (93a:30031) MR.1464198

[Ga] J. B. Garnett, Bounded analytic functions, Pure and Appl. Math., vol. 96, Acad. Press, Inc., New York-London, 1981. MR0628971 (83g:30037)

[Gam] T. Gamelin, Uniform algebras, Prentice-Hall, Inc., Englewood Cliffs, NJ, 1969. MR0410387 $(53: 14137)$

[HJ] V. Havin and B. Jöricke, The uncertainty principle in harmonic analysis, Ergeb. Math. Grenzgeb. (3), vol. 28, Springer-Verlag, Berlin, 1994. MR.1303780 (96c:42001)

[HLP] G. H. Hardy, J. E. Littlewood, and G. Pólya, Inequalities, Cambridge Univ. Press, Cambridge, 1988. MR0944909 (89d:26016)

[HMI] V. Havin and J. Mashreghi, Admissible majorants for model subspaces of $\mathrm{H}^{2}$. I. Slow winding of the generating inner function, Canad. J. Math. 55 (2003), 1231-1263. MR 2016246 (2004i:30029a)

[HMII] Admissible majorants for model subspaces of $\mathrm{H}^{2}$. II. Fast winding of the generating inner function, Canad. J. Math. 55 (2003), 1264-1301. MR2016247 (2004i:30029b)

[Hof] K. Hoffman, Banach spaces of analytic functions, Prentice-Hall, Inc., Englewood Cliffs, NJ, 1962. MR0133008 (24:A2844)

[Koo1] P. Koosis, The logarithmic integral. I, Cambridge Stud. Adv. Math., vol. 12, Cambridge Univ. Press, Cambridge, 1988. MR0961844 (90a:30097)

[Koo2] , The logarithmic integral. II, Cambridge Stud. Adv. Math., vol. 21, Cambridge Univ. Press, Cambridge, 1992. MR1195788 (94i:30027)

[Koo3] Leçons sur le théorème de Beurling et Malliavin, Univ. Montréal, Les Publications CRM, Montréal, QC, 1996. MR1430571 (99e:42023)

[Koo4] - Introduction to $H^{p}$ spaces. With an appendix on Wolff's proof of the corona theorem, London Math. Soc. Lecture Note Ser., vol. 40, Cambridge Univ. Press, Cambridge-New York, 1980. MR0565451 (81c:30062)

[Koo5] Harmonic estimation in certain slit regions and a theorem of Beurling and Malliavin, Acta Math. 142 (1979), 275-305. MR0521462 (80d:31007)

[Koo6] L L L L plus petite majorante surharmonique et son rapport avec l'existence des fonctions entières de type exponentiel jouant le rôle de multiplicateurs, Ann. Inst. Fourier (Grenoble) $\mathbf{3 3}$ (1983), 67-107. MR0698850 (84k:30032)

[Koo7] A local estimate, involving the least superharmonic majorant, for entire functions of exponential type, Algebra i Analiz 10 (1998), no. 3, 45-64; English transl., St. Petersburg Math. J. 10 (1999), no. 3, 441-455. MR:1628022(99g:30035) 
[Koo8] - A result on polynomials and its relation to another concerning entire functions of exponential type, Mat. Fiz. Anal. Geom. 5 (1998), no. 1/2, 68-86. (English) MR1631826 (99d:30005)

[KooP] P. Koosis and H. L. Pedersen, Lower bounds on the values of an entire function of exponential type at certain integers, in terms of a least superharmonic majorant, Algebra i Analiz 10 (1998), no. 3, 31-44; English transl., St. Petersburg Math. J. 10 (1999), no. 3, 429-439. MR1628018 (99g:30034)

[Kr] M. G. KreIn, A contribution to the theory of entire functions of exponential type, Izv. Akad. Nauk SSSR Mat. 11 (1947), no. 4, 309-326. (Russian) MR.0022252 (9:179e)

[L] N. N. Luzin, On a certain integral, Integral and Trigonometric Series, GITTL, Moscow, 1951, pp. 287-319. (Russian) MR0048364(14:2g)

[Ls] N. Levinson, Gap and density theorems, Amer. Math. Soc. Colloq. Publ., vol. 26, Amer. Math. Soc., New York, 1940. MR0003208 (2:180d)

[Lv] B. Ya. Levin, Distribution of zeros of entire functions, Gostekhizdat, Moscow, 1956; English transl., Transl. Math. Monogr., vol. 5, Amer. Math. Soc., Providence, RI, 1980. MR0087740 (19:402 c) MR0589888 (81k:30011)

[MGLP] B. M. Makarov, A. A. Lodkin, A. N. Podkorytov, and M. G. Goluzina, Selected problems in real analysis, "Nevskiı̌ Dialekt," St. Petersburg, 2004. (Russian).

[MIT] G. G. Magaril-Il'yaev and V. M. Tikhomirov, On inequalities for derivatives of Kolmogorov type, Mat. Sb. 188 (1997), no. 12, 73-106; English transl., Sb. Math. 188 (1997), no. 12, 1799-1832. MR1607438 (98m:41022)

[MP] N. Makarov and A. Poltoratski, Meromorphic inner functions, Toeplitz kernels, and the uncertainty principle (to appear).

[Mal] P. Malliavin, On the multiplier theorem for Fourier transforms of measures with compact support, Ark. Mat. 17 (1979), 69-81. MR0543504 (81e:42023)

[N] N. Nikol'skiı̌, Treatise on the shift operator. Spectral function theory, Grundlehren Math. Wiss., vol. 273, Springer-Verlag, Berlin, 1986. MR0827223 (87i:47042)

[P] H. L. Pedersen, Entire functions and logarithmic sums over nonsymmetric sets of the real line, Ann. Acad. Sci. Fenn. Math. 25 (2000), 351-388. MR1762422 (2001f:30034)

[Pr] I. I. Privalov, Boundary properties of analytic functions, 2nd ed., GITTL, Moscow-Leningrad, 1950. (Russian) MR0047765 (13:926h)

[Rem] Ch. Remling, Schrödinger operators and de Branges spaces, J. Funct. Anal. 196 (2002), 323394. MR:1943095 (2003j:47055)

$[\mathrm{Ru}] \quad$ W. Rudin, Fourier analysis on groups, Intersci. Tracts Pure Appl. Math., No. 12, Intersci. Publ., New York-London, 1962. MR0152834 (27:2808)

[T] V. M. Tikhomirov, Some questions in approximation theory, Moskov. Univ., Moscow, 1976. (Russian) MR0487161 (58:6822)

[Ti] E. Titchmarsh, Introduction to the theory of Fourier integrals, Gostekhizdat, MoscowLeningrad, 1948; English transl., Chelsea Publ. Co., New York, 1986. MR0942661 (89c:42002)

[Wor] H. Woracek, de Branges spaces of entire functions closed under forming difference quotients, Integral Equations Operator Theory 37 (2000), 238-249. MR1769812 (2001g:46058)

Département de Mathématiques et de Statistique, Université Laval, Laval, Québec G1K7P4, CANADA

E-mail address: Javad.Mashreghi@mat.ulaval.ca

Department of Mathematics, Michigan State University, East Lansing, Michigan 48821

E-mail address: fedja@math.msu.edu

Department of Mathematics and Mechanics, St. Petersburg State University, Universitetskil Prospect 28, Stary ̌ Peterhof, St. Petersburg 198904, Russia

E-mail address: havin@vH1621.spb.edu

Received 20/MAR/2005

Translated by S. V. KISLYAKOV 